TRANSACTIONS OF THE

AMERICAN MATHEMATICAL SOCIETY

Volume 355, Number 7, Pages 2585-2631

S 0002-9947(03)03141-6

Article electronically published on March 19, 2003

\title{
ALGORITHMS FOR NONLINEAR PIECEWISE POLYNOMIAL APPROXIMATION: THEORETICAL ASPECTS
}

\author{
BORISLAV KARAIVANOV, PENCHO PETRUSHEV, AND ROBERT C. SHARPLEY
}

\begin{abstract}
In this article algorithms are developed for nonlinear $n$-term Courant element approximation of functions in $L_{p}(0<p \leq \infty)$ on bounded polygonal domains in $\mathbb{R}^{2}$. Redundant collections of Courant elements, which are generated by multilevel nested triangulations allowing arbitrarily sharp angles, are investigated. Scalable algorithms are derived for nonlinear approximation which both capture the rate of the best approximation and provide the basis for numerical implementation. Simple thresholding criteria enable approximation of a target function $f$ to optimally high asymptotic rates which are determined and automatically achieved by the inherent smoothness of $f$. The algorithms provide direct approximation estimates and permit utilization of the general Jackson-Bernstein machinery to characterize $n$-term Courant element approximation in terms of a scale of smoothness spaces ( $B$-spaces) which govern the approximation rates.
\end{abstract}

\section{INTRODUCTION}

Highly detailed Digital Terrain Elevation Data (DTED) and associated imagery are now becoming widely available for most of the earth's surface. However, algorithms for effective approximation of data of this type are not yet available. A primary motivation for this work is the development of effective algorithms for nonlinear piecewise polynomial approximation of DTED maps from a redundant hierarchial system over (possibly) irregular triangulations which are constructive in nature. Application of the ideas and theory from [4] to the resulting framework will permit optimal entropy tree encoding of the elevation data, enable progressive view-dependent refinements which may be focused to user-localized regions, and permit the registration of similarly encoded image textures to the surface (see [10, [4] for more details).

Our philosophy is that dependable practical approximation procedures can be built only upon a solid theoretical basis. Accordingly, we have two primary goals in this paper. The first is to better understand nonlinear piecewise polynomial

Received by the editors May 2, 2002.

2000 Mathematics Subject Classification. Primary 41A17, 41A25, 65D18; Secondary 65D07, 42B35.

Key words and phrases. Nested irregular triangulations, redundant representations, nonlinear $n$-term approximation, Courant elements, Jackson and Bernstein estimates.

The second and third authors were supported in part by Grant NSF \#DMS-0079549 and ONR N00014-01-1-0515.

All three authors were supported in part by ONR grant N00014-00-1-0470. 
approximation, in particular, to understand the nature of the global smoothness conditions (spaces) which govern the rate of approximation. The second goal is to develop or refine existing constructive approximation methods for nonlinear approximation which capture the rate of the best approximation and can be implemented effectively in practice.

This paper addresses nonlinear $n$-term approximation by Courant elements generated by multilevel nested triangulations. More precisely, for a given bounded polygonal domain $E \subset \mathbb{R}^{2}$, let $\left(\mathcal{T}_{m}\right)_{m>0}$ be a sequence of triangulations such that each level $\mathcal{T}_{m}$ is a triangulation of $E$ consisting of closed triangles with disjoint interiors and a refinement of the previous level $\mathcal{T}_{m-1}$. We impose some mild natural conditions on the triangulations in order to prevent possible deterioration, but our results are valid for fairly general triangulations with sharp angles. We define $\mathcal{T}:=\bigcup_{m \geq 0} \mathcal{T}_{m}$. Each such multilevel triangulation $\mathcal{T}$ generates a ladder of spaces $\mathcal{S}_{0} \subset \mathcal{S}_{1} \subset \cdots$ consisting of piecewise linear functions, where $\mathcal{S}_{m}(m \geq 0)$ is spanned by all Courant elements $\varphi_{\theta}$ supported on cells $\theta$ at the $m$-th level $\mathcal{T}_{m}$.

Utilizing these primal elements, we consider nonlinear approximation by $n$-term piecewise linear functions of the form $S=\sum_{j=1}^{n} a_{\theta_{j}} \varphi_{\theta_{j}}$, where $\theta_{j}$ may come from different levels and locations. Our first goal is to characterize the approximation spaces consisting of all functions with a given rate of approximation. For approximation in $L_{p}, p<\infty$, this is done in [11, where a collection of smoothness spaces (called $B$-spaces) was introduced and utilized. In this paper, we develop this theory in the more complicated case of approximation in the uniform norm $(p=\infty)$. Our program consists of the following steps. First, in order to quantify the approximation process, we develop a collection of smoothness spaces $B_{\tau}^{\alpha}(\mathcal{T})$ which depend on $\mathcal{T}$ and will govern the best approximation. Second, we prove companion Jackson and Bernstein estimates, and, third, we characterize the approximation spaces by interpolation space methods.

Our second and primary goal is, by using the $B$-spaces and the related techniques, to develop (or refine) algorithms for nonlinear $n$-term Courant element approximation so that the new algorithms are capable of achieving the rate of the best approximation. In the present paper, we develop three such algorithms for $n$-term Courant element approximation in $L_{p}$, which we call "threshold" $(p<\infty)$, "trim and cut" $(0<p \leq \infty)$, and "push the error" $(p=\infty)$ algorithms.

The first step of each of these algorithms is a decomposition step. We denote by $\Theta$ the set of all cells (supports of Courant elements) generated by $\mathcal{T}$. The set $\left(\varphi_{\theta}\right)_{\theta \in \Theta}$ is obviously redundant and, therefore, every function $f$ has infinitely many representations of the form

$$
f=\sum_{\theta \in \Theta} b_{\theta}(f) \varphi_{\theta}
$$

It is crucial to have a sufficiently efficient (sparse) initial representation of the function $f$ that is being approximated. In our case, this means that the representation (1.1) of $f$ should allow a realization of the corresponding $B$-norm $\|f\|_{B_{\tau}^{\alpha}(\mathcal{T})}$. Thus the problem of obtaining an efficient initial representation of the functions is tightly related to the development of the $B$-spaces. We achieve such efficiency by using good projectors into the spaces $\mathcal{S}_{m}, m=0,1, \ldots$

For completeness and comparison, we first consider the natural "threshold" algorithm for $n$-term Courant element approximation, which is valid only in $L_{p}$, $0<p<\infty$. This algorithm simply takes the largest (in $\left.L_{p}\right) n$-terms from (1.1). 
Using the results from [11, it is easy to show that the "threshold" algorithm captures the rate of the best $n$-term Courant element approximation in $L_{p}(p<\infty)$.

The second algorithm, which we call "trim and cut", originates from the proof of the Jackson estimate in [7] and uses the following idea. First, we partition $\Theta$ through a coloring into a family of disjoint trees $\Theta^{\nu}$ (with respect to the inclusion relation): $\Theta:=\bigcup_{\nu=1}^{K} \Theta^{\nu}$. Second, we "trim" each tree by removing cells $\theta \in \Theta^{\nu}$ corresponding to insignificant small terms $a_{\theta} \varphi_{\theta}$ from (1.1), located near the tips of the branches. Third, we divide ("cut") the remaining parts of each tree $\Theta^{\nu}$ into sections of small "energy". Finally, we rewrite the significant part of each section as a linear combination of a small number of Courant elements. The resulting terms determine the final approximant. We shall show that "trim and cut" is capable of achieving the rate of the best approximation in $L_{p}(0<p \leq \infty)$.

Pivotal in our development is the "push the error" algorithm, the name of which was coined by Nira Dyn. The idea for this algorithm appears in [5] and may be roughly described in $L_{\infty}$ as follows. For a fixed $\varepsilon>0$, we "push the error" with $\varepsilon$, starting from the coarsest level $\Theta_{0}$ and proceeding to finer levels. Namely, we denote by $\Lambda_{0}$ the set of all $\theta \in \Theta_{0}$ such that $\left|a_{\theta}\right|>\varepsilon\left(\left\|\varphi_{\theta}\right\|_{\infty}=1\right)$ and define $\mathcal{A}_{0}:=\sum_{\theta \in \Lambda_{0}} a_{\theta} \varphi_{\theta}$. Then we rewrite all remaining terms $a_{\theta} \varphi_{\theta}$ at the next level and add the resulting terms to the existing terms $a_{\theta} \varphi_{\theta}, \theta \in \Theta_{1}$. We denote the new terms by $d_{\theta} \varphi_{\theta}, \theta \in \Theta_{1}$, and select in $\Lambda_{1}$ all $\theta \in \Theta_{1}$ such that $\left|d_{\theta}\right|>\varepsilon$. We continue pushing the error in this way to the finer levels in the representation of $f$. Finally, we define our approximant by $\mathcal{A}:=\sum_{m \geq 0} \mathcal{A}_{j}$. Thus terms $d_{\theta} \varphi_{\theta}$ with $\left|d_{\theta}\right| \leq \varepsilon$ are discarded only at a very fine level, and hence the error (in $L_{\infty}$ ) is $\leq \varepsilon$.

Of course, this naive "push the error" algorithm cannot achieve the rate of the best approximation. However, as we shall show in $\S 3.3$ and $\S 5$, after some substantial improvements, the algorithm is capable of achieving the rate of convergence of the best $n$-term Courant element approximation in the uniform norm.

A focal point of our development is the characterization of the approximation spaces generated by the best $n$-term Courant element approximation in $L_{\infty}$ and the characterization of certain approximation spaces associated with the three algorithms developed, which show that they capture the rate of convergence of the best approximation.

The outline of the paper is as follows. In $\S 2$, we collect all facts needed regarding multilevel triangulations, local approximation, quasi-interpolants, and $B$-spaces. In $\S 3$, we develop and explore the three algorithms for nonlinear $n$-term Courant element approximation: "threshold" algorithm (in §3.1), "trim and cut" algorithm (in $\S 3.2$ ), and "push the error" algorithm (in $\S 3.3$ ). Section 4 is devoted to establishing Jackson and Bernstein inequalities in order to study best $n$-term Courant element approximation. In $\S 5$, we show that the three algorithms capture the rate of the best $n$-term Courant element approximation and identify the associated approximation spaces as $B$-spaces. In $\S 6$, we discuss some of the main issues of nonlinear Courant element approximation. We postpone until the Appendix the proof of an important coloring lemma used in $\S 3.2$ for tree approximation in the "trim and cut" algorithm.

For convenience, we use the convention that positive constants are denoted by $c, c_{1}, \ldots$ throughout and they may vary at every occurrence. The notation $A \approx B$ means that $c_{1} A \leq B \leq c_{2} A$. 


\section{Preliminaries}

In this section we collect all the facts needed regarding multilevel triangulations, local approximation, quasi-interpolants, and other results which were developed in [11] and earlier papers. The essentials are presented for clarity but without proofs.

2.1. Triangulations. By definition $E \subset \mathbb{R}^{2}$ is a bounded polygonal domain if $E$ can be represented as the union of a finite set $\mathcal{T}_{0}$ of closed triangles with disjoint interiors: $E=\bigcup_{\Delta \in \mathcal{T}_{0}} \Delta$. We shall always assume that there exists an initial triangulation $\mathcal{T}_{0}$ of $E$ of this form. We call

$$
\mathcal{T}=\bigcup_{m=0}^{\infty} \mathcal{T}_{m}
$$

a multilevel triangulation of $E$ with levels $\left(\mathcal{T}_{m}\right)$ if the following conditions are fulfilled:

(a) Every level $\mathcal{T}_{m}$ is a partition (or triangulation) of $E$, that is, $E=$ $\bigcup_{\Delta \in \mathcal{T}_{m}} \Delta$ and $\mathcal{T}_{m}$ consists of closed triangles with disjoint interiors.

(b) The levels $\left(\mathcal{T}_{m}\right)$ of $\mathcal{T}$ are nested, i.e., $\mathcal{T}_{m+1}$ is a refinement of $\mathcal{T}_{m}$.

(c) Each triangle $\Delta \in \mathcal{T}_{m}$ has at least two and at most $M_{0}$ children (subtriangles) in $\mathcal{T}_{m+1}$, where $M_{0} \geq 4$ is a constant.

(d) The valence $N_{v}$ of each vertex $v$ of any triangle $\Delta \in \mathcal{T}_{m}$ (the number of the triangles from $\mathcal{T}_{m}$ that share $v$ as a vertex) is at most $N_{0}$, where $N_{0} \geq 3$ is a constant.

(e) No-hanging-vertices condition: No vertex of any triangle $\Delta \in \mathcal{T}_{m}$ that belongs to the interior of $E$ lies in the interior of an edge of another triangle from $\mathcal{T}_{m}$.

We denote by $\mathcal{V}_{m}$ the set of all vertices of triangles from $\mathcal{T}_{m}$, where if $v \in \mathcal{V}_{m}$ is on the boundary of $E$, we include in $\mathcal{V}_{m}$ as many copies of $v$ as is its multiplicity. With this understanding, we set $\mathcal{V}=\bigcup_{m \geq 0} \mathcal{V}_{m}$

We now introduce three types of multilevel nested triangulations which will play an essential role in our developments:

- Locally regular triangulations. We call a multilevel triangulation $\mathcal{T}=$ $\bigcup_{m \geq 0} \mathcal{T}_{m}$ of $E$, a compact polygonal domain in $\mathbb{R}^{2}$, a locally regular triangulation, or briefly an LR-triangulation, if $\mathcal{T}$ satisfies the following additional conditions:

(i) There exist constants $0<r<\rho<1\left(r \leq \frac{1}{4}\right)$, such that for each $\Delta \in \mathcal{T}$ and any child $\Delta^{\prime}$ of $\Delta$ that belongs to $\mathcal{T}$,

$$
r|\Delta| \leq\left|\Delta^{\prime}\right| \leq \rho|\Delta| .
$$

(ii) There exists a constant $0<\delta \leq 1$ such that for each $\Delta^{\prime}, \Delta^{\prime \prime} \in \mathcal{T}_{m}(m \geq 0)$ with a common vertex,

$$
\delta \leq \frac{\left|\Delta^{\prime}\right|}{\left|\Delta^{\prime \prime}\right|} \leq \frac{1}{\delta}
$$

- Strong locally regular triangulations. We call a multilevel triangulation $\mathcal{T}=\bigcup_{m>0} \mathcal{T}_{m}$ of $E$, a compact polygonal domain in $\mathbb{R}^{2}$, a strong locally regular triangulation, or simply an SLR-triangulation, if $\mathcal{T}$ satisfies condition (2.2) and also the following condition (which replaces (2.3)): 
(iii) Affine transform angle condition: There exists a constant $\beta=\beta(\mathcal{T})>$ $0\left(0<\beta<\frac{\pi}{3}\right)$ such that if $\Delta_{0} \in \mathcal{T}_{m}(m \geq 0)$ and $A: \mathbb{R}^{2} \rightarrow \mathbb{R}^{2}$ is an affine transform mapping $\Delta_{0}$ one-to-one, onto an equilateral reference triangle, then for every triangle $\Delta \in \mathcal{T}_{m}$ with a common vertex with $\Delta_{0}$, we have

$$
\text { min angle }(A(\Delta)) \geq \beta
$$

where $A(\Delta)$ is the image of $\Delta$ under $A$ and is therefore also a triangle.

- Regular triangulations. By definition a multilevel triangulation $\mathcal{T}$ of $E \subset \mathbb{R}^{2}$ is called a regular triangulation if $\mathcal{T}$ satisfies the following condition:

(iv) There exists a constant $\beta=\beta(\mathcal{T})>0$ such that the minimal angle of each $\Delta \in \mathcal{T}$ is greater than or equal to $\beta$.

The remainder of this subsection makes several observations to better understand the nature of multilevel triangulations. First, it is clear that the classes of LR- and SLR-triangulations are each invariant under affine transforms. We next observe that each SLR-triangulation is an LR-triangulation, but that the converse statement does not hold. Moreover, each regular triangulation is an SLR-triangulation, but again the converse is in general false. Counterexamples are given in [11.

Each type of triangulation depends on several parameters which are not completely independent. For instance, the parameters of LR-triangulations are $M_{0}, N_{0}$, $r, \rho, \delta$, and $\# \mathcal{T}_{0}$ (the cardinality of $\mathcal{T}_{0}$ ). We could set $M_{0}=\frac{1}{r}, \rho=1-r$ and eliminate these as parameters, but this would tend to obscure the actual dependence of the estimates upon given triangulations.

We next briefly describe a simple standard procedure for constructing multilevel triangulations. We start from an initial triangulation $\mathcal{T}_{0}$ of the given compact polygonal domain $E \subset \mathbb{R}^{2}$. We then select a point on each edge of every triangle $\Delta \in \mathcal{T}_{0}$ and join them within $\Delta$ by edges to subdivide $\Delta$ into four children. The collection of all such children becomes the first generation of triangles, which we denote by $\mathcal{T}_{1}$. We recursively refine in this way to produce succeeding generations $\mathcal{T}_{2}, \mathcal{T}_{3}, \ldots$ The resulting collection $\mathcal{T}:=\bigcup_{m \geq 0} \mathcal{T}_{m}$ is a multilevel triangulation of E.

It is important to know how the quantities $|\Delta|$, min angle $(\Delta)$, and max edge $(\Delta)$ of a triangle $\Delta \in \mathcal{T}$ may change as $\Delta$ moves away from a fixed triangle $\Delta^{\diamond}$ within the same level or through the nested refinements. Consider the case when $\mathcal{T}$ is an LR-triangulation. Then conditions (i) and (ii) suggest a geometric rate of change of $|\Delta|$ (at the same level). In fact, the rate is polynomial [11. Furthermore, if $\Delta^{\prime}, \Delta^{\prime \prime} \in \mathcal{T}_{m}(m \geq 1)$ have a common vertex and are also children of some $\Delta \in \mathcal{T}_{m-1}$, then, as shown in [11, it is possible for $\Delta^{\prime}$ to be equilateral (or close to such), but for $\Delta^{\prime \prime}$ to have an uncontrollably sharp angle (see Figure 1).

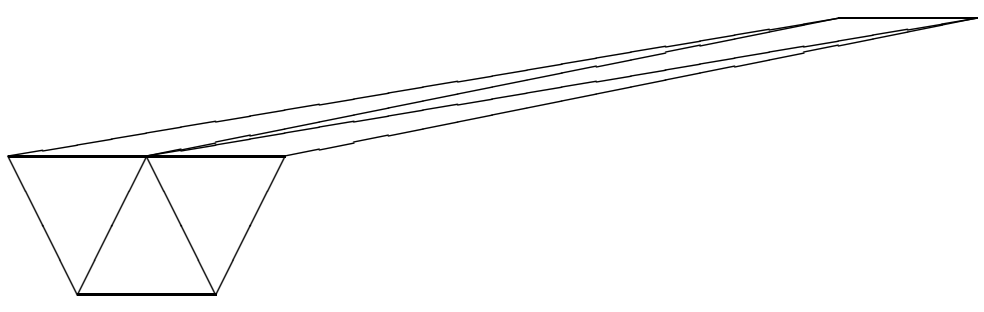

Figure 1. A skewed cell 
If $\mathcal{T}$ is an SLR-triangulation, the above configuration is impossible, but the triangles from $\mathcal{T}$ still may have uncontrollably sharp angles. In this case, min angle $(\Delta)$ changes gradually from one triangle to the adjacent ones.

For any vertex $v \in \mathcal{V}_{m}(m \geq 0)$, we denote by $\theta_{v}$ the cell at level $m$ associated with $v$, i.e., $\theta_{v}$ is the union of all triangles from $\mathcal{T}_{m}$ that have $v$ as a common vertex. We denote by $\Theta_{m}$ the set of all such cells $\theta_{v}$ with $v \in \mathcal{V}_{m}$, and set $\Theta=\bigcup_{m \geq 0} \Theta_{m}$.

2.2. Local piecewise linear approximation and quasi-interpolants. We denote by $\Pi_{k}$ the set of all algebraic polynomials of total degree less than $k$. We shall often refer to the following lemma (see [11]), which establishes the equivalence of different norms of polynomials over different sets.

Lemma 2.1. Let $P \in \Pi_{k}, k \geq 1$, and $0<p, q \leq \infty$.

(a) For any triangle $\Delta \subset \mathbb{R}^{2}$,

$$
\|P\|_{L_{p}(\Delta)} \approx|\Delta|^{\frac{1}{p}-\frac{1}{q}}\|P\|_{L_{q}(\Delta)} .
$$

(b) If $\Delta$ and $\Delta^{\prime}$ are two triangles such that $\Delta^{\prime} \subset \Delta$ and $|\Delta| \leq c_{1}\left|\Delta^{\prime}\right|$, then

$$
\|P\|_{L_{p}(\Delta)} \leq c\|P\|_{L_{p}\left(\Delta^{\prime}\right)} .
$$

(c) If $\Delta^{\prime} \subset \Delta$ and $\left|\Delta^{\prime}\right| \leq c_{1}|\Delta|$ with $0<c_{1}<1$, then

$$
\|P\|_{L_{p}(\Delta)} \leq c\|P\|_{L_{p}\left(\Delta \backslash \Delta^{\prime}\right)} \approx|\Delta|^{\frac{1}{p}-\frac{1}{q}}\|P\|_{L_{q}\left(\Delta \backslash \Delta^{\prime}\right)} .
$$

In the above expressions, the constants depend at most on the corresponding parameters and the constant $c_{1}$.

The no-hanging-vertices condition (e) of triangulations guarantees the existence of Courant elements. Namely, for any vertex $v \in \mathcal{V}_{m}(m \geq 0)$ there exists a unique Courant element $\varphi_{\theta_{v}}$ supported on $\theta_{v} \in \Theta_{m}$ which is the unique continuous piecewise linear function on $E$ that is supported on $\theta_{v}$ and satisfies $\varphi_{\theta_{v}}(v)=1$. We denote $\Phi:=\Phi_{\mathcal{T}}:=\left(\varphi_{\theta}\right)_{\theta \in \Theta}$. We also denote by $\mathcal{S}_{m}$ the space of all continuous piecewise linear functions over $\mathcal{T}_{m}$. Clearly, $S \in \mathcal{S}_{m}$ if and only if $S=\sum_{v \in \mathcal{V}_{m}} S(v) \varphi_{\theta_{v}}$. Throughout the remainder of this section, we assume that $\mathcal{T}$ is an LR-triangulation of $E$. We shall often use the following stability estimates for $\left(\varphi_{\theta}\right)_{\theta \in \Theta_{m}}$.

Lemma 2.2. Let $0<q \leq \infty$ and $S=\sum_{\theta \in \Theta_{m}} a_{\theta} \varphi_{\theta}, m \geq 0$, with coefficients $a_{\theta} \in \mathbb{R}$. Then for every $\Delta \in \mathcal{T}_{m}$, we have

$$
\|S\|_{L_{q}(\Delta)} \approx\left(\sum_{\theta \in \Theta_{m}, \theta \supset \Delta}\left\|a_{\theta} \varphi_{\theta}\right\|_{q}^{q}\right)^{\frac{1}{q}}
$$

and hence

$$
\|S\|_{L_{q}(E)} \approx\left(\sum_{\theta \in \Theta_{m}}\left\|a_{\theta} \varphi_{\theta}\right\|_{q}^{q}\right)^{\frac{1}{q}}
$$

with constants of equivalence depending only on the parameters of $\mathcal{T}$. In these estimates the $\ell_{q}$-norm is replaced by the sup-norm if $q=\infty$.

The proof of this lemma is fairly simple and can be found in [11.

- Local piecewise linear approximation. The local approximation by continuous piecewise linear functions will be an important tool in our further development. 
For $f \in L_{\eta}(E), \eta>0$, and any $\Delta \in \mathcal{T}_{m}(m \geq 0)$, we denote the error of $L_{\eta}\left(\Omega_{\Delta}\right)$ approximation to $f$ from $\mathcal{S}_{m}$ by

$$
S S_{\Delta}(f)_{\eta}:=S S_{\Delta}(f, \mathcal{T})_{\eta}:=\inf _{S \in \mathcal{S}_{m}}\|f-S\|_{L_{\eta}\left(\Omega_{\Delta}\right)},
$$

where $\Omega_{\Delta}$ is the union of all triangles from $\mathcal{T}_{m}$ that have a vertex in common with $\Delta$.

- Quasi-interpolants. The set $\Phi_{\mathcal{T}}$ of all Courant elements is obviously redundant. To obtain a good (i.e., sparse) representation of a given function $f$, we shall use the following well-known quasi-interpolant:

$$
Q_{m}(f):=Q_{m}(f, \mathcal{T}):=\sum_{\theta \in \Theta_{m}}\left\langle f, \tilde{\varphi}_{\theta}\right\rangle \varphi_{\theta}
$$

where $\langle f, g\rangle:=\int_{E} f g$ and $\left(\tilde{\varphi}_{\theta}\right)$ are the duals of $\left(\varphi_{\theta}\right)$ defined by

$$
\tilde{\varphi}_{\theta}:=\sum_{\Delta \in \mathcal{T}_{m}, \Delta \subset \theta} \mathbb{1}_{\Delta} \tilde{\lambda}_{\Delta, \theta}
$$

with $\tilde{\lambda}_{\Delta, \theta}$ the linear polynomial that is equal to $\frac{9}{N_{v}|\Delta|}$ at $v_{\theta}$, the "central vertex" of $\theta$, and equal to $-\frac{3}{N_{v} \mid \Delta}$ at the other two vertices of $\Delta$ (recall that $N_{v}$ is the valence of $v$ ). It is easily seen that

$$
\left\langle\varphi_{\theta}, \tilde{\varphi}_{\theta^{\prime}}\right\rangle=\delta_{\theta \theta^{\prime}}, \text { for } \theta, \theta^{\prime} \in \Theta_{m} .
$$

Obviously, $Q_{m}$ is a linear projector, i.e., $Q_{m}(S)=S$ for $S \in \mathcal{S}_{m}$. It is crucial that $\tilde{\varphi}_{\theta} \in L_{\infty}$ and $\tilde{\varphi}_{\theta}$ is locally supported. Consequently, $Q_{m}$ is locally bounded and provides good local approximation.

Lemma 2.3. (a) If $f \in L_{\eta}(E), 1 \leq \eta \leq \infty$, and $\Delta \in \mathcal{T}_{m}, m \geq 0$, then

$$
\left\|Q_{m}(f)\right\|_{L_{\eta}(\Delta)} \leq c\|f\|_{L_{\eta}\left(\Omega_{\Delta}\right)} .
$$

(b) If $0<\eta \leq \infty$ and $g=\sum_{\Delta \in \mathcal{T}_{m}} \mathbb{1}_{\Delta} \cdot P_{\Delta}$ with $P_{\Delta} \in \Pi_{2}$ and $m \geq 0$, then

$$
\left\|Q_{m}(g)\right\|_{L_{\eta}(\Delta)} \leq c\|g\|_{L_{\eta}\left(\Omega_{\Delta}\right)}, \quad \text { for } \Delta \in \mathcal{T}_{m}
$$

The constants above depend only on $\eta$ and the parameters of $\mathcal{T}$.

For a proof of this lemma, see [11].

From the above lemma, we see that $Q_{m}: L_{\eta}(E) \rightarrow \mathcal{S}_{m}(1 \leq \eta \leq \infty)$ is a locally bounded linear projector. There is a well-known scheme for extending $Q_{m}$ to a nonlinear projector $Q_{m}: L_{\eta}(E) \rightarrow \mathcal{S}_{m}$ for $0<\eta<1$. This is needed for nonlinear approximation in $L_{p}(0<p \leq 1)$. To describe this extension, let $P_{\Delta, \eta}: L_{\eta}(\Delta) \rightarrow \Pi_{2}$ $(0<\eta \leq \infty)$ be a projector (linear if $\eta \geq 1$ and nonlinear if $0<\eta<1$ ) such that

$$
\left\|f-P_{\Delta, \eta}(f)\right\|_{L_{\eta}(\Delta)} \leq c E_{2}(f, \Delta) \quad \text { for } f \in L_{\eta}(\Delta),
$$

where $E_{2}(f, \Delta)$ is the error of the best $L_{\eta}(\Delta)$-approximation to $f$ from $\Pi_{2}$ (the linear polynomials). We define

$$
p_{m, \eta}(f):=\sum_{\Delta \in \mathcal{T}_{m}} \mathbb{1}_{\Delta} \cdot P_{\Delta, \eta}(f)
$$

and set

$$
T_{m, \eta}(f):=Q_{m}\left(p_{m, \eta}(f)\right), \quad \text { for } f \in L_{\eta}(E)
$$


Clearly, $T_{m, \eta}: L_{\eta}(E) \rightarrow \mathcal{S}_{m}$ is a projector (linear if $\eta \geq 1$ and nonlinear if $0<\eta<1)$.

The next lemma, established in [11, shows that $Q_{m}$ and $T_{m, \eta}$ provide good local approximations from $\mathcal{S}_{m}$.

Lemma 2.4. (a) If $f \in L_{\eta}(E), 1 \leq \eta \leq \infty$, and $\Delta \in \mathcal{T}_{m}, m \geq 0$, then

$$
\left\|f-Q_{m}(f)\right\|_{L_{\eta}(\Delta)} \leq c S S_{\Delta}(f)_{\eta} .
$$

(b) If $f \in L_{\eta}(E), 0<\eta \leq \infty$, and $\Delta \in \mathcal{T}_{m}, m \geq 0$, then

$$
\left\|f-T_{m}(f)\right\|_{L_{\eta}(\Delta)} \leq c S S_{\Delta}(f)_{\eta} .
$$

The constants above depend only on $\eta$ and the parameters of $\mathcal{T}$.

The needed convergence of $Q_{m}(f)$ and $T_{m}(f)$ to $f$ is provided by the following result (see Lemma 2.15 from [11]).

Lemma 2.5. If $f \in L_{\eta}(E)$, then

$$
\begin{aligned}
\left\|f-Q_{m}(f)\right\|_{L_{\eta}(E)} \rightarrow 0 \text { as } m \rightarrow \infty, & \text { if } 1 \leq \eta \leq \infty, \\
\left\|f-T_{m, \eta}(f)\right\|_{L_{\eta}(E)} \rightarrow 0 \text { as } m \rightarrow \infty, & \text { if } 0<\eta \leq \infty .
\end{aligned}
$$

Now, we apply a well-known scheme for obtaining sparse Courant element representation of functions. We define

$$
q_{m}:=Q_{m}-Q_{m-1} \text { and } t_{m, \eta}:=T_{m, \eta}-T_{m-1, \eta}, \text { for } m \geq 0,
$$

where $Q_{-1}:=0$ and $T_{-1, \eta}:=0$. Clearly, $q_{m}(f), t_{m, \eta}(f) \in \mathcal{S}_{m}$.

For a given function $f \in L_{\eta}(E), 1 \leq \eta \leq \infty$, we define the sequence $\mathbf{b}(f):=$ $\left(b_{\theta}(f)\right)_{\theta \in \Theta_{m}}$ from the expression

$$
q_{m}(f)=: \sum_{\theta \in \Theta_{m}} b_{\theta}(f) \varphi_{\theta}, \quad m \geq 0 .
$$

Using Lemma 2.5, we have

$$
f=\sum_{m \geq 0} q_{m}(f)=\sum_{m \geq 0} \sum_{\theta \in \Theta_{m}} b_{\theta}(f) \varphi_{\theta} \quad \text { in } L_{\eta} .
$$

If $f \in L_{\eta}(E), 0<\eta<1$, we define the sequence $\mathbf{b}_{\eta}(f):=\left(b_{\theta, \eta}(f)\right)_{\theta \in \Theta_{m}}$ by

$$
t_{m, \eta}(f)=: \sum_{\theta \in \Theta_{m}} b_{\theta, \eta}(f) \varphi_{\theta}, \quad m \geq 0,
$$

and again by Lemma 2.5 we have

$$
f=\sum_{m \geq 0} t_{m, \eta}(f)=\sum_{m \geq 0} \sum_{\theta \in \Theta_{m}} b_{\theta, \eta}(f) \varphi_{\theta} \quad \text { in } L_{\eta} .
$$

Clearly, $\mathbf{b}(\cdot)$ is a linear operator while $\mathbf{b}_{\eta}(\cdot)(0<\eta<1)$ is nonlinear. 
2.3. $B$-spaces. In this section, we include the necessary tools for the $B$-spaces which we need for nonlinear $n$-term Courant element approximation. The $B$-spaces over multilevel nested triangulations of $\mathbb{R}^{2}$ are introduced in [11] and used there for nonlinear $n$-term Courant element approximation in $L_{p}\left(\mathbb{R}^{2}\right)(0<p<\infty)$. In the present paper, we shall use the $B$-spaces for $n$-term Courant element approximation in $L_{p}(E)(0<p \leq \infty)$, where $E$ is a compact polygonal domain in $\mathbb{R}^{2}$. We shall put the emphasis on approximation in the uniform norm $(p=\infty)$. There are three types of $B$-spaces (skinny, slim, and fat $B$-spaces) that were introduced in 11 to serve different purposes. For Courant element approximation, we need the slim $B$-spaces, which we shall simply call $B$-spaces.

Throughout this paper, we assume that $\mathcal{T}$ is an $L R$-triangulation of a compact polygonal domain $E$ in $\mathbb{R}^{2}$. Moreover, the $B$-spaces $B_{\tau}^{\alpha}(\mathcal{T})$, with parameter set $1 / \tau:=\alpha+1 / p$ according to two specific choices: (a) $p=\infty$ and $\alpha \geq 1$; or (b) $0<p<\infty$ and $\alpha>0$, will arise naturally in our algorithms and error estimates. These spaces have several equivalent definitions, which we briefly describe.

- Definition of $B_{\tau}^{\alpha}(\mathcal{T})$ via local approximation. We define $B_{\tau}^{\alpha}(\mathcal{T})$ as the set of all functions $f \in L_{\tau}(E)$ such that

$$
|f|_{B_{\tau}^{\alpha}(\mathcal{T})}:=\left(\sum_{\Delta \in \mathcal{T}}\left(|\Delta|^{-\alpha} S S_{\Delta}(f)_{\tau}\right)^{\tau}\right)^{1 / \tau}<\infty,
$$

where $S S_{\Delta}(f)_{\tau}$ is the error of $L_{\tau}\left(\Omega_{\Delta}\right)$-approximation (local) to $f$ from $\mathcal{S}_{m}$ for $\Delta \in \mathcal{T}_{m}$ (see (2.5) ). It is readily seen that $|f+g|_{B_{\tau}^{\alpha}}^{\tau^{*}} \leq|f|_{B_{\tau}^{\alpha}}^{\tau^{*}}+|g|_{B_{\tau}^{\alpha}}^{\tau^{*}}$ with $\tau^{*}:=$ $\min \{\tau, 1\}$, and $|f+s|_{B_{\tau}^{\alpha}}=|f|_{B_{\tau}^{\alpha}}$ for $s \in \mathcal{S}_{0}$. Hence $|\cdot|_{B_{\tau}^{\alpha}}$ is a semi-norm if $\tau \geq 1$ and a semi-quasi-norm if $\tau<1$.

By Theorems 2.7 and 2.9 below, it follows that if $f \in B_{\tau}^{\alpha}(\mathcal{T})$, then $f \in L_{p}(E)$. Therefore, it is natural to define a (quasi-)norm in $B_{\tau}^{\alpha}(\mathcal{T})$ by

$$
\|f\|_{B_{\tau}^{\alpha}(\mathcal{T})}:=\|f\|_{p}+|f|_{B_{\tau}^{\alpha}(\mathcal{T})} .
$$

More generally, for $0<\eta<p$, we define

$$
N_{S S, \eta}(f, \mathcal{T}):=\|f\|_{p}+\left(\sum_{\Delta \in \mathcal{T}}\left(|\Delta|^{1 / p-1 / \eta} S S_{\Delta}(f)_{\eta}\right)^{\tau}\right)^{1 / \tau} .
$$

Evidently, $N_{S S, \tau}(f, \mathcal{T})=\|f\|_{B_{\tau}^{\alpha}(\mathcal{T})}$. When clear from the context, we use $N_{\mathbb{S}, \tau}(f)$.

- Definition of norm in $\boldsymbol{B}_{\tau}^{\alpha}(\mathcal{T})$ via atomic decomposition. For $f \in L_{\tau}(E)$, we define

$$
N_{\Phi}(f):=\inf _{f=\sum_{\theta \in \Theta} c_{\theta} \varphi_{\theta}}\left(\sum_{\theta \in \Theta}\left(|\theta|^{-\alpha}\left\|c_{\theta} \varphi_{\theta}\right\|_{\tau}\right)^{\tau}\right)^{1 / \tau},
$$

where the infimum is taken over all representations $f=\sum_{\theta \in \Theta} c_{\theta} \varphi_{\theta}$ in $L_{\tau}(E)$. Note that the existence of such representations of $f$ follows by (2.11) and (2.13). By Theorem 2.7 below,

$$
\sum_{\theta \in \Theta}\left(|\theta|^{-\alpha}\left\|c_{\theta} \varphi_{\theta}\right\|_{\tau}\right)^{\tau}<\infty \quad \text { implies }\left\|\sum_{\theta \in \Theta}\left|c_{\theta} \varphi_{\theta}(\cdot)\right|\right\|_{p}<\infty,
$$

and hence $f \in L_{p}(E)$ and the series $\sum_{\theta \in \Theta}\left|c_{\theta} \varphi_{\theta}(\cdot)\right|$ converges a.e. and in $L_{p}(E)$. Therefore, the way in which the terms of the series are ordered is not essential, 
and the convergence in $L_{\tau}(E)$ implies a stronger (absolute) convergence in $L_{p}(E)$ $(\tau<p)$. By Lemma 2.1, it follows that

$$
\begin{aligned}
N_{\Phi}(f) & \approx \inf _{f=\sum_{\theta \in \Theta} c_{\theta} \varphi_{\theta}}\left(\sum_{\theta \in \Theta}\left(|\theta|^{1 / p}\left|c_{\theta}\right|\right)^{\tau}\right)^{1 / \tau} \\
& \approx \inf _{f=\sum_{\theta \in \Theta} c_{\theta} \varphi_{\theta}}\left(\sum_{\theta \in \Theta}\left\|c_{\theta} \varphi_{\theta}\right\|_{p}^{\tau}\right)^{1 / \tau} .
\end{aligned}
$$

If $p=\infty$, then

$$
N_{\Phi}(f) \approx \inf _{f=\sum_{\theta \in \Theta} c_{\theta} \varphi_{\theta}}\left(\sum_{\theta \in \Theta}\left|c_{\theta}\right|^{\tau}\right)^{1 / \tau}
$$

- Definition of norms in $\boldsymbol{B}_{\tau}^{\alpha}(\mathcal{T})$ via projectors. For $f \in L_{\eta}(E)$, we let

$$
f=\sum_{\theta \in \Theta} b_{\theta, \eta}(f) \varphi_{\theta}
$$

be the representation of $f$ from (2.11) if $\eta \geq 1$ and from (2.13) if $0<\eta<1$. We define

$$
N_{Q, \tau}(f):=\left(\sum_{\theta \in \Theta}\left(|\theta|^{-\alpha}\left\|b_{\theta, \tau}(f) \varphi_{\theta}\right\|_{\tau}\right)^{\tau}\right)^{1 / \tau}
$$

and, more generally (in accordance with (2.16)),

$$
N_{Q, \eta}(f):=\left(\sum_{\theta \in \Theta}\left(|\theta|^{1 / p-1 / \eta}\left\|b_{\theta, \eta}(f) \varphi_{\theta}\right\|_{\eta}\right)^{\tau}\right)^{1 / \tau} .
$$

By Lemmas 2.1 and 2.2, we have

$$
\begin{gathered}
N_{Q, \eta}(f) \approx\left(\sum_{\Delta \in \mathcal{T}}\left(|\Delta|^{1 / p-1 / \eta}\left\|q_{m}(f)\right\|_{L_{\eta}(\Delta)}\right)^{\tau}\right)^{1 / \tau}, \quad \text { if } \eta \geq 1, \\
N_{Q, \eta}(f) \approx\left(\sum_{\Delta \in \mathcal{T}}\left(|\Delta|^{1 / p-1 / \eta}\left\|t_{m, \eta}(f)\right\|_{L_{\eta}(\Delta)}\right)^{\tau}\right)^{1 / \tau}, \quad \text { if } 0<\eta<1,
\end{gathered}
$$

and

$$
N_{Q, \eta}(f) \approx\left(\sum_{\theta \in \Theta}\left(|\theta|^{1 / p}\left|b_{\theta, \eta}(f)\right|\right)^{\tau}\right)^{1 / \tau} \approx\left(\sum_{\theta \in \Theta}\left\|b_{\theta, \eta}(f) \varphi_{\theta}\right\|_{p}^{\tau}\right)^{1 / \tau} .
$$

In the most interesting case of $p=\infty$,

$$
N_{Q, \eta}(f) \approx\left(\sum_{\theta \in \Theta}\left|b_{\theta, \eta}(f)\right|^{\tau}\right)^{1 / \tau}
$$

- General $B$-spaces. A more general $B$-space $B_{p q}^{\alpha}(\mathcal{T}), \alpha>0,0<p, q \leq \infty$, is defined as the set of all $f \in L_{p}(E)$ such that

$$
\|f\|_{B_{p q}^{\alpha}(\mathcal{T})}:=\inf _{f=\sum_{\theta \in \Theta} c_{\theta} \varphi_{\theta}}\left(\sum_{m \in \mathbb{Z}}\left[2^{m \alpha}\left(\sum_{\theta \in \Theta, 2^{-m} \leq|\theta|<2^{-m+1}}\left\|c_{\theta} \varphi_{\theta}\right\|_{p}^{p}\right)^{1 / p}\right]^{q}\right)^{1 / q}<\infty,
$$


where the $\ell_{q}$-norm is replaced by the sup-norm if $q=\infty$. In this paper, we do not need the $B$-spaces in such generality.

- Embedding theorems and equivalence of norms. We recall our assumptions. We have $0<p \leq \infty$, and $\alpha \geq 1$ if $p=\infty$ and $\alpha>0$ if $p<\infty$. In both cases, $1 / \tau:=\alpha+1 / p(1 / \tau:=\alpha$ if $p=\infty)$. We record estimates and embeddings from [1], along with the necessary modifications, which are necessary for the development of the main results of this paper. The first embedding result appears as Theorem 2.16 in [11.

Theorem 2.6. For $0<\tau<p$ or $p=\infty, \tau \leq 1$, then for any sequence of real numbers $\left(c_{\theta}\right)_{\theta \in \Theta}$, we have

$$
\left\|\sum_{\theta \in \Theta}\left|c_{\theta}\right| \varphi_{\theta}\right\|_{p} \leq c\left(\sum_{\theta \in \Theta}\left\|c_{\theta} \varphi_{\theta}\right\|_{p}^{\tau}\right)^{1 / \tau}
$$

where $c$ depends only on $\tau, p$, and the parameters of $\mathcal{T}$.

Theorem 2.7. If $f \in L_{\eta}(E)$ with $0<\eta<p$, and $N_{Q, \eta}(f)<\infty$, then $f \in L_{p}(E)$ $(f \in C(E)$ if $p=\infty)$, and $f$ has the representation $f=\sum_{\theta \in \Theta} b_{\theta, \eta}(f) \varphi_{\theta}$ with the series converging absolutely a.e. in $E$ and in $L_{p}$ (respectively, in $C(E)$ ), and

$$
\|f\|_{p} \leq\left\|\sum_{\theta \in \Theta}\left|b_{\theta, \eta}(f)\right| \varphi_{\theta}\right\|_{p} \leq c N_{Q, \eta}(f)
$$

where $c$ is independent of $f$.

Proof. For $0<p<\infty$, the result follows from (2.11), (2.13), and Theorem 2.9 below. If $p=\infty$, the theorem follows by (2.11), (2.13), (2.25), and the following estimates:

$$
\left\|\sum_{\theta \in \Theta} b_{\theta, \eta}(f), \varphi_{\theta}\right\|_{\infty} \leq\left(\sum_{\theta \in \Theta}\left|b_{\theta, \eta}(f)\right|^{\tau}\right)^{1 / \tau} \leq c N_{Q, \eta}(f) \quad(\tau=1 / \alpha \leq 1) .
$$

Remark 2.8. It is easily seen that Theorem 2.7 is not true when $p=\infty$ and $\alpha<1$. For this reason we impose the restriction $\alpha \geq 1$ when $p=\infty$ throughout.

Theorem 2.9. The norms $\|\cdot\|_{B_{\tau}^{\alpha}(\mathcal{T})}, N_{S S, \eta}(\cdot)(0<\eta<p), N_{\Phi}(\cdot)$, and $N_{Q, \eta}(\cdot)$ $(0<\eta<p)$, defined in (2.15), (2.16), (2.17), and (2.21), are equivalent with constants of equivalence depending only on $p, \tau, \eta$, and the parameters of $\mathcal{T}$.

Proof. One proceeds exactly as in [1] (see the proof of Theorem 2.17 of that reference) and proves that

$$
\begin{aligned}
|f|_{B_{\tau}^{\alpha}(\mathcal{T})} \approx\left(\sum_{\Delta \in \mathcal{T}}\left(|\Delta|^{1 / p-1 / \eta} S S_{\Delta}(f)_{\eta}\right)^{\tau}\right)^{1 / \tau} & \approx \inf _{f=\sum_{\theta \in \Theta} c_{\theta} \varphi_{\theta}}\left(\sum_{\theta \in \Theta \backslash \Theta_{0}}\left\|c_{\theta} \varphi_{\theta}\right\|_{p}^{\tau}\right)^{1 / \tau} \\
(2.28) & \approx\left(\sum_{\theta \in \Theta \backslash \Theta_{0}}\left\|b_{\theta, \eta}(f) \varphi_{\theta}\right\|_{p}^{\tau}\right)^{1 / \tau}
\end{aligned}
$$

provided $0<\eta<p$. To obtain the norm estimate from these semi-norm equivalences, we use Theorem 2.6 to give $\|f\|_{p} \leq c N_{\Phi}(f)$. Using this, (2.28), and the remark after the definition of $N_{\Phi}(f)$ in (2.17), we obtain

$$
\|f\|_{B_{\tau}^{\alpha}(\mathcal{T})} \approx N_{S S, \eta}(f) \leq c N_{\Phi}(f) \leq c N_{Q, \eta}(f), \quad 0<\eta<p .
$$


For the reverse inequality, we use Lemma 2.2, Theorem [2.6] and (2.28) to obtain

$$
\begin{aligned}
\left(\sum_{\theta \in \Theta_{0}}\left\|b_{\theta, \eta}(f) \varphi_{\theta}\right\|_{p}^{\tau}\right)^{1 / \tau} & \leq c\left(\# \Theta_{0}, \tau, p\right)\left(\sum_{\theta \in \Theta_{0}}\left\|b_{\theta, \eta}(f) \varphi_{\theta}\right\|_{p}^{p}\right)^{1 / p} \\
& \leq c\left\|\sum_{\theta \in \Theta_{0}} b_{\theta, \eta}(f) \varphi_{\theta}\right\|_{p} \leq c\left(\|f\|_{p}+\left\|\sum_{\theta \in \Theta \backslash \Theta_{0}} b_{\theta, \eta}(f) \varphi_{\theta}\right\|_{p}\right) \\
& \leq c\|f\|_{p}+c\left(\sum_{\theta \in \Theta \backslash \Theta_{0}}\left\|b_{\theta, \eta}(f) \varphi_{\theta}\right\|_{p}^{\tau}\right)^{1 / \tau} \\
& \leq c\|f\|_{B_{\tau}^{\alpha}(\mathcal{T}) .}
\end{aligned}
$$

This and (2.28) imply $N_{Q, \eta}(f) \leq c\|f\|_{B_{\tau}^{\alpha}(\mathcal{T})}$.

The next embedding theorem of Sobolev type follows immediately from (2.18) or (2.24).

Theorem 2.10. For $0<\alpha_{0}<\alpha_{1}$ and $\tau_{j}:=\left(\alpha_{j}+1 / p\right)^{-1}, j=0,1$, we have the continuous embedding

$$
B_{\tau_{1}}^{\alpha_{1}}(\mathcal{T}) \subset B_{\tau_{0}}^{\alpha_{0}}(\mathcal{T})
$$

i.e., if $f \in B_{\tau_{1}}^{\alpha_{1}}(\mathcal{T})$, then $f \in B_{\tau_{0}}^{\alpha_{0}}(\mathcal{T})$ and $\|f\|_{B_{\tau_{0}}^{\alpha_{0}}(\mathcal{T})} \leq c\|f\|_{B_{\tau_{1}}^{\alpha_{1}}(\mathcal{T})}$.

- Interpolation. We first recall some basic definitions from the real interpolation method. We refer the reader to [2] and [1] as general references for interpolation theory. For a pair of quasi-normed spaces $X_{0}, X_{1}$, embedded in a Hausdorff space, the space $X_{0}+X_{1}$ is defined as the collection of all functions $f$ that can be represented as $f_{0}+f_{1}$ with $f_{0} \in X_{0}$ and $f_{1} \in X_{1}$. The quasi-norm in $X_{0}+X_{1}$ is defined by

$$
\|f\|_{X_{0}+X_{1}}:=\|f\|_{X_{0}+X_{1}}+\inf _{f=f_{0}+f_{1}}\left\|f_{0}\right\|_{X_{0}}+\left\|f_{1}\right\|_{X_{1}} .
$$

The $K$-functional is defined for each $f \in X_{0}+X_{1}$ and $t>0$ by

$$
K(f, t):=K\left(f, t ; X_{0}, X_{1}\right):=\inf _{f=f_{0}+f_{1}}\left\|f_{0}\right\|_{X_{0}}+t\left\|f_{1}\right\|_{X_{1}} .
$$

The real interpolation space $\left(X_{0}, X_{1}\right)_{\lambda, q}$ with $0<\lambda<1$ and $0<q \leq \infty$ is defined as the set of all $f \in X_{0}+X_{1}$ such that

$$
\|f\|_{\left(X_{0}, X_{1}\right)_{\lambda, q}}:=\left(\int_{0}^{\infty}\left(t^{-\lambda} K(f, t)\right)^{q} \frac{d t}{t}\right)^{1 / q}<\infty,
$$

where the $L_{q}$-norm is replaced by the sup-norm if $q=\infty$.

It is easily seen that if $X_{1} \subset X_{0}\left(X_{1}\right.$ continuously embedded in $\left.X_{0}\right)$, then $K(f, t) \approx\|f\|_{X_{0}}$ for $f \in X_{0}$ and $t \geq 1$, and, consequently,

$$
\|f\|_{\left(X_{0}, X_{1}\right)_{\lambda, q}} \approx\|f\|_{X_{0}}+\left(\sum_{\nu=0}^{\infty}\left[2^{\nu \lambda} K\left(f, 2^{-\nu}\right)\right]^{q}\right)^{1 / q} .
$$

Theorem 2.11. Suppose $0<p \leq \infty$ and further assume that both $\alpha_{0}, \alpha_{1} \geq 1$ in the case $p=\infty$, and $\alpha_{0}, \alpha_{1}>0$ otherwise. Furthermore, let $\tau_{j}:=\left(\alpha_{j}+1 / p\right)^{-1}$, $j=0,1$. Then

$$
\left(B_{\tau_{0}}^{\alpha_{0}}(\mathcal{T}), B_{\tau_{1}}^{\alpha_{1}}(\mathcal{T})\right)_{\lambda, \tau}=B_{\tau}^{\alpha}(\mathcal{T})
$$


with equivalent norms, provided $\alpha=(1-\lambda) \alpha_{0}+\lambda \alpha_{1}$ with $0<\lambda<1$ and $\tau:=$ $(\alpha+1 / p)^{-1}$.

Proof. We shall prove (2.32) only in the case $p>1$. For a proof of (2.32) when $p \leq 1$, see [3].

We shall use the abbreviated notation $B^{\alpha}:=B_{\tau}^{\alpha}(\mathcal{T})$ and $B^{\alpha_{j}}:=B_{\tau_{j}}^{\alpha_{j}}(\mathcal{T}), j=$ 0,1 . Also, we denote by $\ell_{q}$ the space of all sequences $\mathbf{a}=\left(a_{\theta}\right)_{\theta \in \Theta}$ of real numbers such that $\|\mathbf{a}\|_{\ell_{q}}:=\left(\sum_{\theta \in \Theta}\left|a_{\theta}\right|^{q}\right)^{1 / q}<\infty$.

We set $\eta:=1$ and normalize the Courant elements in $L_{p}$, that is, $\left\|\varphi_{\theta}\right\|_{p}=1$. We also renormalize the duals $\tilde{\varphi}_{\theta}$ from (2.7) accordingly. We denote again by $\mathbf{b}(f)=\left(b_{\theta}\right)_{\theta \in \Theta}$ the sequence from (2.10) with respect to the normalized Courant elements. By (2.24), Theorem 2.7, and Theorem 2.9. if $f \in B^{\alpha_{j}}, j=0,1$, then

$$
f=\sum_{\theta \in \Theta} b_{\theta}(f) \varphi_{\theta} \quad \text { and } \quad\|f\|_{B^{\alpha_{j}}} \approx\|\mathbf{b}(f)\|_{\ell_{\tau_{j}}},
$$

recalling that the elements $\varphi_{\theta}$ are normalized in $L_{p}$. The corresponding statement holds for functions $f \in B^{\alpha}$ as well.

We shall next employ the following interpolation theorem (see, e.g., $\S 5.1$ of [1] or [2]) which follows directly from the definition of the $K$-functional and the norms of the interpolation spaces. Suppose $T$ is a linear operator which boundedly maps $X_{0}$ into $Y_{0}$ and $X_{1}$ into $Y_{1}$, where $\left(X_{0}, X_{1}\right)$ and $\left(Y_{0}, Y_{1}\right)$ are couples of quasi-normed spaces as above. Then for $0<\lambda<1$ and $0<q \leq \infty, T$ boundedly maps $\left(X_{0}, X_{1}\right)_{\lambda, q}$ into $\left(Y_{0}, Y_{1}\right)_{\lambda, q}$.

We introduce linear operators $\mathcal{I}$ and $\mathcal{P}$ as follows: $\mathcal{I}$ is defined by $\mathcal{I}(f)_{\theta}:=b_{\theta}(f)$, $\theta \in \Theta$, and $\mathcal{P}$ is given by $\mathcal{P}(\mathbf{a}):=\sum_{\theta \in \Theta} a_{\theta} \varphi_{\theta}, \mathbf{a}=\left(a_{\theta}\right)_{\theta \in \Theta}$. By (2.33), $\|\mathbf{b}(f)\|_{\ell_{\tau_{j}}} \leq$ $c\|f\|_{B^{\alpha_{j}}}$ for $f \in B^{\alpha_{j}}, j=0,1$, and hence $\mathcal{I}: B^{\alpha_{j}} \rightarrow \ell_{\tau_{j}}$ (boundedly). By the above-mentioned interpolation theorem,

$$
\mathcal{I}:\left(B^{\alpha_{0}}, B^{\alpha_{1}}\right)_{\lambda, \tau} \rightarrow\left(\ell_{\tau_{0}}, \ell_{\tau_{1}}\right)_{\lambda, \tau} \quad \text { (boundedly). }
$$

Similarly, if $\mathbf{a} \in \ell_{\tau_{j}}$, then by Theorems 2.7 and 2.9. we may conclude that $\mathcal{P}(\mathbf{a}) \stackrel{L_{p}}{=}$ $\sum_{\theta \in \Theta} a_{\theta} \varphi_{\theta}$ is well defined. So if we set $f=\mathcal{P}(\mathbf{a})$, then

$$
\|\mathcal{P}(\mathbf{a})\|_{B^{\alpha_{j}}} \leq c \inf _{f=\sum_{\theta \in \Theta} c_{\theta} \varphi_{\theta}}\left\|\left(c_{\theta}\right)_{\theta \in \Theta}\right\|_{\ell_{\tau_{j}}} \leq c\|\mathbf{a}\|_{\ell_{\tau_{j}}}, \quad j=0,1 .
$$

Thus $\mathcal{P}: \ell_{\tau_{j}} \rightarrow B^{\alpha_{j}}$ (boundedly), and by interpolation

$$
\mathcal{P}:\left(\ell_{\tau_{0}}, \ell_{\tau_{1}}\right)_{\lambda, \tau} \rightarrow\left(B^{\alpha_{0}}, B^{\alpha_{1}}\right)_{\lambda, \tau} \quad \text { (boundedly). }
$$

Finally, we recall the well-known interpolation result (see, e.g., [2], [1]):

$$
\left(\ell_{\tau_{0}}, \ell_{\tau_{1}}\right)_{\lambda, \tau}=\ell_{\tau}, \quad \text { where } \frac{1}{\tau}=\frac{1-\lambda}{\tau_{0}}+\frac{\lambda}{\tau_{1}} \text { with } 0<\lambda<1 .
$$

Clearly, (2.32) follows by (2.33)-(2.36).

- Skinny $B$-spaces. The skinny $B$-spaces were introduced in [11] and used for characterization of nonlinear (discontinuous) piecewise polynomial approximation on $\mathbb{R}^{2}$. We next adapt that definition to the case of approximation on a compact polygonal domain $E \subset \mathbb{R}^{2}$. Suppose $\mathcal{T}$ is a multilevel nested triangulation of $E$ which additionally satisfies condition (2.2) (see $\S 2.1$ and [11]). The skinny $B$-space 
$\mathcal{B}_{\tau}^{\alpha k}(\mathcal{T})$, where $k \geq 1$ and $\alpha$ and $\tau$ are as above, is defined as the set of all $f \in L_{\tau}(E)$ such that

$$
|f|_{\mathcal{B}_{\tau}^{\alpha k}(\mathcal{T})}:=\left(\sum_{\Delta \in \mathcal{T}}\left(|\Delta|^{-\alpha} \omega_{k}(f, \Delta)_{\tau}\right)^{\tau}\right)^{1 / \tau}<\infty,
$$

where $\omega_{k}(f, \Delta)_{\tau}$ is a $k$ th modulus of smoothness of $f$ in $L_{\tau}(\Delta)$, defined by

$$
\omega_{k}(f, \Delta)_{\tau}:=\sup _{h \in \mathbb{R}^{2}}\left\|\Delta_{h}^{k}(f, \cdot)\right\|_{L_{\tau}(\Delta)}
$$

and $\Delta_{h}^{k}(f, \cdot)$ is the $k$ th difference of $f$. The norm in $\mathcal{B}_{\tau}^{\alpha k}(\mathcal{T})$ is defined by $\|\cdot\|_{\mathcal{B}_{\tau}^{\alpha k}(\mathcal{T})}:=\|\cdot\|_{p}+|\cdot|_{\mathcal{B}_{\tau}^{\alpha k}(\mathcal{T})}$

- Fat $B$-spaces: The link to Besov spaces. Suppose $\mathcal{T}$ is an SLR-triangulation of a compact polygonal domain $E \subset \mathbb{R}^{2}$. Similarly as in 11], we define the fat $B$ space $\mathbb{B}_{\tau}^{\alpha k}(\mathcal{T})$, where $k \geq 1$ and $\alpha$ and $\tau$ are as above, as the set of all functions $f \in L_{\tau}(E)$ such that

$$
|f|_{\mathbb{B}_{\tau}^{\alpha k}(\mathcal{T})}:=\left(\sum_{\Delta \in \mathcal{T}}\left(|\Delta|^{-\alpha} \omega_{k}\left(f, \Omega_{\Delta}\right)_{\tau}\right)^{\tau}\right)^{1 / \tau}<\infty .
$$

We endow $\mathbb{B}_{\tau}^{\alpha k}(\mathcal{T})$ with the norm $\|\cdot\|_{\mathbb{B}_{\tau}^{\alpha k}(\mathcal{T})}:=\|\cdot\|_{p}+|\cdot|_{\mathbb{B}_{\tau}^{\alpha k}(\mathcal{T})}$. Using Whitney's theorem, it readily follows that $c_{1} \omega_{2}(f, \Delta)_{\tau} \leq S S_{\Delta}(f)_{\tau} \leq c_{2} \omega_{2}\left(f, \Omega_{\Delta}\right)_{\tau}$, and hence $|f|_{\mathcal{B}_{\tau}^{\alpha 2}(\mathcal{T})} \leq c|f|_{B_{\tau}^{\alpha}(\mathcal{T})} \leq c|f|_{\mathbb{B}_{\tau}^{\alpha 2}(\mathcal{T})}$. The space $\mathbb{B}_{\tau}^{\alpha 2}(\mathcal{T})$ is a natural candidate to replace $B_{\tau}^{\alpha}(\mathcal{T})$ in nonlinear $n$-term Courant element approximation. This is, however, only possible for sufficiently small $\alpha\left(0<\alpha<\alpha_{0}\right)$. Otherwise $\mathbb{B}_{\tau}^{\alpha 2}(\mathcal{T})$ is too "fat" and cannot do the job. Finally, we note that if $\mathcal{T}$ is a regular triangulation and $0<\alpha<k$, then $\mathbb{B}_{\tau}^{\alpha k}(\mathcal{T})$ coincides with the Besov space $B_{\tau}^{2 \alpha}\left(L_{\tau}\right)$. For a more complete discussion of this and other related issues, see [11].

\section{Algorithms for $n$-Term Courant approximation}

- Decomposition step for all approximation algorithms. The first step of each of the three approximation algorithms that we consider in this section is a decomposition step. This step is not trivial, since the set $\Phi_{\mathcal{T}}:=\left(\varphi_{\theta}\right)_{\theta \in \Theta}$ of all Courant elements is redundant and, therefore, each function has infinitely many representations using Courant elements. For each algorithm, it is crucial to have a sufficiently efficient initial representation of the function $f$ that is being approximated. This means that the representation of $f$ should allow a realization of the corresponding $B$-norm.

To construct the initial representation, we consider two cases of metric approximation. If the approximation takes place in $L_{p}, 1<p \leq \infty$, we utilize the decomposition of $f$ via quasi-interpolation from (2.11) with $1 \leq \eta<p$, while if $0<p \leq 1$, we use (2.13) with $0<\eta<p$. In both cases, we have an initial desirable sparse representation of $f$ of the form

$$
f=\sum_{\theta \in \Theta} b_{\theta} \varphi_{\theta}, \quad b_{\theta}=b_{\theta}(f)
$$

which allows a realization of the $B$-norm (see (2.24)-(2.25), and Theorem 2.9). For the remainder of this section, in order to more easily track the dependency of the 
constants appearing in the inequalities, we redefine $\|f\|_{B_{\tau}^{\alpha}(\mathcal{T})}$ by

$$
\|f\|_{B_{\tau}^{\alpha}(\mathcal{T})}:=\left(\sum_{\theta \in \Theta}\left(\left|b_{\theta} \| \theta\right|^{1 / p}\right)^{\tau}\right)^{1 / \tau} \approx\left(\sum_{\theta \in \Theta}\left\|b_{\theta} \varphi_{\theta}\right\|_{p}^{\tau}\right)^{1 / \tau}
$$

which is an equivalent norm in $B_{\tau}^{\alpha}(\mathcal{T})$ (see Theorem 2.9). Without loss of generality, we may assume (when needed) that there is a final level $\Theta_{L}(L<\infty)$ in (3.1).

3.1. "Threshold" algorithm ( $\boldsymbol{p}<\infty$ only). In this algorithm we utilize the usual thresholding strategy used for $n$-term approximation from a basis in $L_{p}(1<$ $p<\infty)$. The resulting procedure performs extremely well, due to the sparse representation realized by the first step. We note, however, that the derived error estimates involve constants that depend on $p$ and become unbounded as $p \rightarrow \infty$. The "push the error" and "trim and cut" algorithms described later in this section will be shown to achieve the corresponding estimates for the uniform norm $(p=\infty)$. For this subsection we therefore assume that $f \in L_{p}, 0<p<\infty$.

\section{- Description of the "threshold" algorithm.}

Step 1. (Decompose) We use the decomposition of $f \in L_{p}(E)$ from (3.1).

Step 2. (Select the $n$ largest terms) We order the terms $\left(b_{\theta} \varphi_{\theta}\right)_{\theta \in \Theta}$ in a sequence $\left(b_{\theta_{j}} \varphi_{\theta_{j}}\right)_{j=1}^{\infty}$ so that

$$
\left\|b_{\theta_{1}} \varphi_{\theta_{1}}\right\|_{p} \geq\left\|b_{\theta_{2}} \varphi_{\theta_{2}}\right\|_{p} \geq \cdots .
$$

Then we define the approximant $A_{n}^{T}(f)_{p}$ by $A_{n}^{T}(f)_{p}:=\sum_{j=1}^{n} b_{\theta_{j}} \varphi_{\theta_{j}}$.

- Error estimation for the "threshold" algorithm. We denote the corresponding error of approximation of this threshold algorithm by

$$
\mathbb{A}_{n}^{T}(f)_{p}:=\left\|f-A_{n}^{T}(f)_{p}\right\|_{p}
$$

The argument used in establishing the Jackson error estimate in [11] may be modified in obvious ways to prove the following error estimate.

Theorem 3.1. If $f \in B_{\tau}^{\alpha}(\mathcal{T}), \alpha>0,1 / \tau:=\alpha+1 / p(0<p<\infty)$, then

$$
\mathbb{A}_{n}^{T}(f)_{p} \leq c n^{-\alpha}\|f\|_{B_{\tau}^{\alpha}(\mathcal{T})},
$$

where $c$ depends on $\alpha, p$, and the parameters of $\mathcal{T}$.

In $\S 5$, we shall need the following result:

Lemma 3.2. If $f=\sum_{\theta \in \Theta} b_{\theta} \varphi_{\theta}$ is the decomposition of $f$ from (3.1), then

$$
\mathbb{A}_{2 n}^{T}(f)_{p} \leq c n^{-\alpha}\left(\sum_{j=n+1}^{\infty}\left\|b_{\theta_{j}} \varphi_{\theta_{j}}\right\|_{p}^{\tau}\right)^{1 / \tau},
$$

where $\left(b_{\theta_{j}} \varphi_{\theta_{j}}\right)_{j=1}^{\infty}$ is as in Step 2 and $c$ depends on $\alpha, p$, and the parameters of $\mathcal{T}$.

Proof. Applying Theorem 3.4 from [1] to $\left(b_{\theta_{j}} \varphi_{\theta_{j}}\right)_{j=n+1}^{\infty}$ immediately provides the desired result.

Remark 3.3. As we have mentioned, the main drawback of the "threshold" algorithm is that it is not applicable to approximation in the uniform norm, since the constant $c=c(\alpha, p)$ in (3.4) tends to infinity as $p \rightarrow \infty$ and the performance of the algorithm deteriorates as $p$ gets large. The obvious reason for this behavior is that 
$f$ can be built out of many terms $\left(b_{\theta} \varphi_{\theta}\right)$ which have small coefficients and are supported at the same location. These terms can pile up to an essential contribution, but the algorithm will fail to anticipate their future significance.

3.2. "Trim and cut (the tree)" algorithm. The idea of this algorithm has its origins in the proof of the Jackson estimate in [7] (see §5, pages 272-276). The approximation considered there is by wavelets or splines over a uniform partition in the uniform norm. We shall refine this idea to develop an algorithm for $n$-term Courant element approximation in $L_{p}(E), 0<p \leq \infty$, over LR-triangulations. We begin with a brief description of the algorithm and then elaborate on the details of each of the main steps.

\section{- Description of the "trim and cut" algorithm.}

Step 1. (Decompose). We use the common decomposition of $f \in L_{p}(E)$ given in (3.1).

Step 2. (Organize the cells of $\Theta$ into manageable trees $\Theta^{\nu}$ ). We develop an algorithm (procedure) for coloring the cells of $\Theta$ in such a way that the cells of the same color form a tree structure as described in Lemma 3.4 below. This organization greatly simplifies the management of the estimates, both the approximation construction and the enumeration of "active" Courant elements in our approximant.

Step 3. (Trim each tree). Since all the elements may initially affect the $B$ space norm of a function, we need to preprocess each tree by pruning all branches which may have many leaves, but do not make a significant contribution to the norm of the function $f$. We do this by running a stopping time argument from the finest level to a coarser level, until a significant cumulative contribution is met. We prune the branch just below that element.

Step 4. (Partition the remaining trees into "segments"). We continue to partition the remainders of each of the $K$ trees by cutting them at each of the joins of branches to form chains from the tree. We will easily be able to track the number of chains produced by this procedure. A second stopping time argument is then applied to cut the chains into "segments" in order to control the number of significant elements added to the approximant (at most $N_{0}+1$ from each segment) and to guarantee that the cumulative effect of the left-over elements (i.e., error) can be controlled by the final Step 5 .

Step 5. (Rewrite the "segments" to control error). Here each segment is rewritten at its finest level, and its terminal element (with the new coefficients) and some of its neighboring elements are added to the approximant. This allows for a void to be created, so that the residual of the segment will have disjoint support with all remaining segments as well as the residuals of those previously processed. This insures that the cumulative pointwise error remains under control.

We now describe these rather vague steps in more detail. Step 1 is clear from our earlier discussion.

Step 2. In the following lemma, we construct a procedure for coloring the elements of $\Theta$ with $K$ colors $\nu$, so that no two Courant elements of the same color from the 
same level have supports that intersect; in fact, corresponding cells of the same color will have a tree structure with set inclusion as the order relation. This allows us to partition $\Theta$ into a disjoint union of sets $\Theta^{\nu}(1 \leq \nu \leq K)$, and correspondingly organize $f$ as the sum $f=\sum_{\nu=1}^{K} f_{\nu}$, where $f_{\nu}:=\sum_{\theta \in \Theta^{\nu}} b_{\theta} \varphi_{\theta}$. We can then proceed to process each of the $f_{\nu}$ without worrying about its terms from the same level overlapping, and at worst a factor of $K$ will come into the constants for the estimates that we derive. For its proof, see the Appendix.

Lemma 3.4 (Coloring lemma). For any multilevel-triangulation $\mathcal{T}$ of $E$, the set $\Theta:=\Theta(\mathcal{T})$ of all cells generated by $\mathcal{T}$ can be represented as a finite disjoint union of its subsets $\left(\Theta^{\nu}\right)_{\nu=1}^{K}$ with $K=K\left(N_{0}, M_{0}\right)\left(N_{0}\right.$ is the maximal valence and $M_{0}$ is the maximal number of children of a triangle in $\mathcal{T}$ ) such that each $\Theta^{\nu}$ has a tree structure with respect to set inclusion, i.e., if $\theta^{\prime}, \theta^{\prime \prime} \in \Theta^{\nu}$ with $\left(\theta^{\prime}\right)^{\circ} \cap\left(\theta^{\prime \prime}\right)^{\circ} \neq \emptyset$, then either $\theta^{\prime} \subset \theta^{\prime \prime}$ or $\theta^{\prime \prime} \subset \theta^{\prime}$.

In order to complete the remaining Steps 3-5 we must consider two variations in the details of the algorithm, depending on whether $p=\infty$ or $0<p<\infty$. The case of the uniform metric is presented in Subsection 3.2.1, while the case of $L_{p}$ $(0<p<\infty)$ is given in Subsection 3.2.2.

3.2.1. The case $p=\infty$. Fix $\varepsilon>0$ and let $\varepsilon^{*}:=\frac{\varepsilon}{2 K}$, where we recall that $K$ is the number of colors representing the tree structures.

Step 3. Trimming of $\Theta^{\nu}(1 \leq \nu \leq K)$ with $\varepsilon^{*}$. We trim each $\Theta^{\nu}$, starting from the finest level $\Theta_{J}^{\nu}$ and proceeding to the coarsest level. We remove from $\Theta^{\nu}$ every cell $\theta^{\diamond}$ such that

$$
\sum_{\theta \subset \theta^{\diamond}}\left|b_{\theta}\right| \leq \varepsilon^{*}
$$

We denote by $\Gamma^{\nu}$ the set of all $\theta \in \Theta^{\nu}$ that have been retained after completing this procedure, and by $\Gamma_{f}^{\nu}$ the set of all final cells in $\Gamma^{\nu}$, i.e., $\theta^{\diamond} \in \Gamma_{f}^{\nu}$ iff there is no $\theta \in \Gamma^{\nu}$ such that $\theta \subsetneq \theta^{\diamond}$. Clearly, for each $\theta^{\diamond} \in \Gamma_{f}^{\nu}$,

$$
\sum_{\theta \subset \theta^{\prime}}\left|b_{\theta}\right| \leq \varepsilon^{*} \text { for each } \theta^{\prime} \subsetneq \theta^{\diamond} \text {, but } \sum_{\theta \subset \theta^{\diamond}}\left|b_{\theta}\right|>\varepsilon^{*} \text {. }
$$

We denote $f_{\Gamma^{\nu}}:=\sum_{\theta \in \Gamma^{\nu}} b_{\theta} \varphi_{\theta}$. Therefore,

$$
\left\|f_{\nu}-f_{\Gamma^{\nu}}\right\|_{\infty} \leq \max _{\theta^{\diamond} \notin \Gamma^{\nu}}\left\|\sum_{\theta \subset \theta^{\diamond}} b_{\theta} \varphi_{\theta}\right\|_{\infty} \leq \max _{\theta^{\diamond} \notin \Gamma^{\nu}} \sum_{\theta \subset \theta^{\diamond}}\left|b_{\theta}\right| \leq \varepsilon^{*}
$$

and hence, if we set $f_{\Gamma}:=\sum_{\nu=1}^{K} f_{\Gamma^{\nu}}$, then

$$
\left\|f-f_{\Gamma}\right\|_{\infty} \leq K \varepsilon^{*}=\varepsilon / 2 .
$$

Step 4. Partitioning the branches of each tree $\Gamma^{\nu}$ into chains and the chains into "segments". For each of the tree structures $\Gamma^{\nu}(1 \leq \nu \leq K)$, we denote by $\Gamma_{b}^{\nu}$ the set of all branching cells in $\Gamma^{\nu}$ (cells with more than one child in $\Gamma^{\nu}$ ) and by $\Gamma_{c h}^{\nu}$ the set of all chain cells in $\Gamma^{\nu}$ (cells with exactly one child in $\Gamma^{\nu}$ ). It is easy to see that

$$
\# \Gamma_{b}^{\nu} \leq \# \Gamma_{f}^{\nu}
$$

In fact, one proceeds by induction from the finest to coarser levels, associating each branch cell from $\Gamma_{b}^{\nu}$ by a cell from $\Gamma_{f}^{\nu}$. For each branch cell, there is always at least 
one member of $\Gamma_{f}^{\nu}$ still available from each descendant edge. Only one is used to associate with the current branch cell, thereby leaving at least one available for its next ancestor branch cell in that line.

On the other hand, $\# \Gamma_{c h}^{\nu}$ may be much larger than $\# \Gamma_{f}^{\nu}$, and so we will need to process these elements. A collection of cells $\theta_{1} \supset \theta_{2} \supset \cdots \supset \theta_{l}$ is called a chain if for $j=1, \ldots, l-1, \theta_{j+1}$ is a child of $\theta_{j}$ and $\theta_{j} \in \Gamma_{c h}^{\nu}$, and the terminal cell $\theta_{l} \in \Gamma_{f}^{\nu} \cup \Gamma_{b}^{\nu}$. We partition the tree $\Gamma^{\nu}$ into chains. Namely, we start at the coarsest level and construct (maximal) chains which will terminate with either a final cell (in $\Gamma_{f}^{\nu}$ ) or a branching cell (in $\Gamma_{b}^{\nu}$ ). We continue this procedure to the finest level.

We next "section" each chain into segments using $\varepsilon^{*}$ as a threshold. Namely, if $\lambda$ is a chain and $\lambda=\left(\theta_{j}\right)_{j=1}^{l}$ with $\theta_{1} \supset \theta_{2} \supset \cdots \supset \theta_{l}$, then we start from the coarsest element $\theta_{1}$ and sum the coefficients of each cell, moving to the next child of the chain until the sum exceeds the threshold. At this point we cut the chain to form the first (significant) segment and start this procedure again with the next child in line until this is not possible (i.e., ending without the threshold being crossed). We call this type of segment a "remnant segment". Therefore, this procedure cuts $\lambda$ into disjoint segments $\sigma$ of the form $\left(\theta_{j}\right)_{j=i}^{i+\mu}, \mu \geq 0$, so that each segment satisfies exactly one of the following conditions:

(a) $\sigma$ consists of a single "significant cell":

$$
\left|b_{\theta_{i}}\right|>\varepsilon^{*}(\text { case of } \mu=0),
$$

(b) $\sigma$ is a "significant segment":

$$
\sum_{j=i}^{i+\mu-1}\left|b_{\theta_{j}}\right| \leq \varepsilon^{*}, \text { but } \sum_{j=i}^{i+\mu}\left|b_{\theta_{j}}\right|>\varepsilon^{*}(\text { case of } \mu>0)
$$

(c) $\sigma$ is a "remnant segment":

$$
\sum_{j=i}^{l}\left|b_{\theta_{j}}\right| \leq \varepsilon^{*}
$$

We denote by $\Sigma^{\nu}$ the set of all such segments $\sigma=\left(\theta_{j}\right)_{j=i}^{i+\mu}$ resulting from this procedure.

Step 5. Rewriting elements from certain segments of $\Sigma^{\nu}$. Let $\sigma=\left(\theta_{j}\right)_{j=1}^{\mu}$ be any

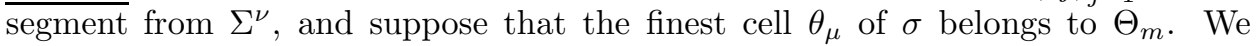
rewrite the Courant elements $\left(\sum_{j=1}^{\mu} b_{\theta_{j}} \varphi_{\theta_{j}}\right)$ of the segment at its finest $(m$-th) level, finding coefficients $\left(c_{\theta}\right)$ such that

$$
\sum_{\theta \in \Theta_{m}, \theta^{\circ} \cap \theta_{\mu} \neq \emptyset} c_{\theta} \varphi_{\theta}=\sum_{j=1}^{\mu} b_{\theta_{j}} \varphi_{\theta_{j}} \text { on } \theta_{\mu} .
$$

We denote $\mathcal{X}_{\sigma}:=\left\{\theta \in \Theta_{m}: \theta^{\circ} \cap \theta_{\mu} \neq \emptyset\right.$ and $\left.\theta \subset \theta_{1}\right\}$. Obviously, if $\mu=1$ (i.e., the segment consists of a single cell), then the coefficient remains unchanged and $\mathcal{X}_{\sigma}=\sigma=\left\{\theta_{1}\right\}$. Observe in any case that $\# \mathcal{X}_{\sigma} \leq N_{0}+1$ and $\bigcup_{\theta \in \mathcal{X}_{\sigma}} \theta \subset \theta_{1}$. Finally, set $\Sigma:=\bigcup_{\nu=1}^{K} \Sigma^{\nu}$, and correspondingly define

$$
A_{\varepsilon}^{T C}(f):=\sum_{\sigma \in \Sigma} \sum_{\theta \in \mathcal{X}_{\sigma}} c_{\theta} \varphi_{\theta}
$$

as our approximant produced by the "trim and cut" algorithm. 
- Error estimation for the "trim and cut" algorithm (case $p=\infty$ ). Suppose that the "trim and cut" procedure has been applied to a function $f$ with $\varepsilon>0$, and $A_{\varepsilon}^{T C}(f)=\sum_{\theta \in \Lambda_{\varepsilon}} c_{\theta} \varphi_{\theta}$ is the resulting approximant from (3.13), where $\Lambda_{\varepsilon}=\bigcup_{\sigma \in \Sigma} \mathcal{X}_{\sigma}$. We denote

$$
n(\varepsilon):=n_{f}(\varepsilon):=\# \Lambda_{\varepsilon}, \quad \mathbb{A}_{n(\varepsilon)}^{T C}(f)_{\infty}:=\left\|f-A_{\varepsilon}^{T C}(f)\right\|_{\infty},
$$

and

$$
\mathbb{A}_{n}^{T C}(f)_{\infty}:=\inf \left\{\mathbb{A}_{n(\varepsilon)}^{T C}(f)_{\infty}: n(\varepsilon) \leq n\right\} .
$$

Note that each of these quantities depend implicitly on $\mathcal{T}$. To complete our results for the "trim and cut" algorithm, we show first in Lemma 3.5 that this is a good approximation to $f$, and then that the number of elements that are used in the approximant satisfies the correct estimates (see Theorem 3.7 below).

Lemma 3.5. Suppose that $A_{\varepsilon}^{T C}(f)$ is the approximant for $f$ given in equation (3.13) which has been constructed using the "trim and cut" algorithm. Then

$$
\left\|f-A_{\varepsilon}^{T C}(f)\right\|_{\infty} \leq \varepsilon .
$$

Proof. Following the definition (3.13) of $A_{\varepsilon}^{T C}(f)$, we define

$$
A^{\nu}:=\sum_{\sigma^{\prime} \in \Sigma^{\nu}} \sum_{\theta \in \mathcal{X}_{\sigma^{\prime}}} c_{\theta} \varphi_{\theta}
$$

Then obviously $A_{\varepsilon}^{T C}(f)=\sum_{\nu=1}^{K} A^{\nu}$. Since $\varepsilon^{*}=\frac{\varepsilon}{2 K}$, it suffices to show that $\left\|f_{\Gamma^{\nu}}-A^{\nu}\right\|_{\infty} \leq \varepsilon^{*}$.

In Step 5 we extracted the heart of each segment $\sigma=\left(\theta_{j}\right)_{j=1}^{\mu}$, added its contribution to the approximant (3.13), and cleared room for descendant cells. To estimate the associated error, we introduce the ring for $\sigma$ as $R_{\sigma}:=\theta_{1} \backslash \theta_{\mu}$; then $R_{\sigma}=\emptyset$ when $\sigma$ consists of a significant cell (i.e., condition (3.10) holds). For any nonempty ring $R_{\sigma}\left(\sigma \in \Sigma^{\nu}\right)$, set $\sigma^{\prime}:=\left(\theta_{j}\right)_{j=1}^{\mu-1}$ and observe that at worst

$$
\begin{aligned}
\left\|f_{\Gamma^{\nu}}-A^{\nu}\right\|_{L_{\infty}\left(R_{\sigma}\right)} & =\left\|\sum_{\theta \in \sigma} b_{\theta} \varphi_{\theta}-\sum_{\theta \in \mathcal{X}_{\sigma}} c_{\theta} \varphi_{\theta}\right\|_{L_{\infty}\left(R_{\sigma}\right)} \\
& \leq\left\|\sum_{\theta \in \sigma^{\prime}} b_{\theta} \varphi_{\theta}\right\|_{L_{\infty}\left(\theta_{1}\right)} \leq \sum_{\theta \in \sigma^{\prime}}\left|b_{\theta}\right| \leq \varepsilon^{*}
\end{aligned}
$$

It is easy to see that all rings $R_{\sigma}\left(\sigma \in \Sigma^{\nu}\right)$ are disjoint and the set where $A^{\nu}$ may differ from $f_{\Gamma^{\nu}}$ is contained in $\bigcup_{\sigma \in \Sigma^{\nu}} R_{\sigma}$. Hence, by summing over all segments $\sigma$ and then over all colors $\nu$, it follows that

$$
\left\|f_{\Gamma}-A_{\varepsilon}^{T C}(f)\right\|_{\infty} \leq \sum_{\nu=1}^{K} \sum_{\sigma \in \Sigma^{\nu}}\left\|f_{\Gamma^{\nu}}-A^{\nu}\right\|_{L_{\infty}\left(R_{\sigma}\right)} \leq K \varepsilon^{*}=\frac{\varepsilon}{2} .
$$

This together with estimate (3.8) implies the desired error estimate (3.14).

Remark 3.6. Conditions (3.5), (3.11), and (3.12) can be relaxed by replacing every $\operatorname{sum} \sum\left|b_{\theta}\right|$ by $\left\|\sum b_{\theta} \varphi_{\theta}\right\|_{\infty}$. This would not change the rate of approximation, but may improve the constants in a practical implementation. 
Theorem 3.7. If $f \in B_{\tau}^{\alpha}(\mathcal{T}), \alpha \geq 1, \tau:=1 / \alpha$, then for each $\varepsilon>0$,

$$
\mathbb{A}_{n(\varepsilon)}^{T C}(f)_{\infty} \leq \varepsilon \text { and } n(\varepsilon) \leq c \varepsilon^{-\tau}\|f\|_{B_{\tau}^{\alpha}(\mathcal{T})}^{\tau},
$$

where $c=c\left(N_{0}, M_{0}, \alpha\right)$. Therefore,

$$
\mathbb{A}_{n}^{T C}(f)_{\infty} \leq c n^{-\alpha}\|f\|_{B_{\tau}^{\alpha}(\mathcal{T})} .
$$

Proof. We have already shown in Lemma 3.5 that $\mathbb{A}_{n(\varepsilon)}^{T C}(f)_{\infty} \leq \varepsilon$; so we only need to establish $n(\varepsilon) \leq c \varepsilon^{-\tau}\|f\|_{B_{\tau}^{\alpha}(\mathcal{T})}^{\tau}$. We first observe that it is enough to estimate $\# \Sigma^{\nu}$, since contributions to the approximant occur only as each segment from $\Sigma^{\nu}$ is processed. Note that at most one element is contributed for segments consisting of a single significant cell (3.10) and at most $N_{0}+1$ contributions for the segments satisfying instead either (3.11) or (3.12).

In order to estimate $\# \Sigma^{\nu}$ we first estimate $\# \Gamma_{f}^{\nu}$, since it will estimate certain terms. The stopping criterium (3.6) in Step 3,

$$
\varepsilon^{*}<\sum_{\theta \subseteq \theta^{\diamond}}\left|b_{\theta}\right|,
$$

must hold for each $\theta^{\diamond} \in \Gamma_{f}^{\nu}$. So if we apply the $\tau$-th power to both sides, use the embedding of the sequence spaces $(\tau \leq 1)$, sum over all $\theta \in \Gamma_{f}^{\nu}$, and observe that the supports of the cells in $\Gamma_{f}^{\nu}$ have disjoint interiors, then we obtain

$$
\# \Gamma_{f}^{\nu}\left(\varepsilon^{*}\right)^{\tau}<\sum_{\theta^{\diamond} \in \Gamma_{f}^{\nu}} \sum_{\theta \subseteq \theta^{\diamond}}\left|b_{\theta}\right|^{\tau} \leq\|f\|_{B_{\tau}^{\alpha}(\mathcal{T})}^{\tau} .
$$

The rightmost inequality follows immediately by our definition of the norm of $B_{\tau}^{\alpha}(\mathcal{T})($ see $(3.2))$.

To complete the proof of the theorem, we only need to establish a similar estimate for the number of elements of $\Sigma^{\nu}$. Recall, however, that the segments $\sigma$ are formed as disjoint segments of cells from the tree structure and come as one of two types, $\Sigma_{\text {sig }}$, those exceeding the threshold (see conditions (3.10) or (3.11)) and, $\Sigma_{\text {rem }}$, those that do not (see condition (3.12)). From the construction it follows that remnant segments terminate with either a unique final cell or a unique branching cell, and so by (3.9),

$$
\# \Sigma_{\text {rem }} \leq \# \Gamma_{b}^{\nu}+\# \Gamma_{f}^{\nu} \leq 2 \# \Gamma_{f}^{\nu},
$$

which has just been shown in 3.19 to satisfy the desired bound.

Therefore we are reduced to estimating $\# \Sigma_{\text {sig. }}$. But the same idea used in estimating $\# \Gamma_{f}^{\nu}$ (see (3.18)-3.19) $)$ may be employed once again. Indeed, we just replace the condition (3.18) with

$$
\varepsilon^{*}<\sum_{\theta \in \sigma}\left|b_{\theta}\right|
$$

and use the fact that the segments are disjoint (considered as part of the tree structure), in order to obtain

$$
\# \Sigma_{\text {sig }}\left(\varepsilon^{*}\right)^{\tau}<\sum_{\sigma \in \Sigma_{\text {sig }}} \sum_{\theta \in \sigma}\left|b_{\theta}\right|^{\tau} \leq\|f\|_{B_{\tau}^{\alpha}(\mathcal{T})}^{\tau} .
$$

Although not required here, the following lemma will be needed in $\S 5$ and can now be established using the techniques of this section. 
Lemma 3.8. Let $f=f^{0}+f^{1}$, where $f=\sum_{\theta \in \Theta} b_{\theta} \varphi_{\theta}, f^{j}=\sum_{\theta \in \Theta} b_{\theta}^{j} \varphi_{\theta}(j=0,1)$ with $b_{\theta}=b_{\theta}^{0}+b_{\theta}^{1}($ all $\theta \in \Theta)$, and let

$$
\mathcal{N}_{j}:=\left(\sum_{\theta \in \Theta}\left|b_{\theta}^{j}\right|^{\tau_{j}}\right)^{1 / \tau_{j}}<\infty \quad(j=0,1)
$$

with $\alpha_{j} \geq 1$ and $\tau_{j}=1 / \alpha_{j}$. If the "trim and cut" algorithm with $\varepsilon=\varepsilon_{0}+\varepsilon_{1}$ $\left(\varepsilon_{j}>0\right)$ has been applied to $f$, represented as above in place of Step 1 , then

$$
\begin{gathered}
\mathbb{A}_{n\left(\varepsilon_{0}+\varepsilon_{1}\right)}^{T C}(f)_{\infty} \leq \varepsilon_{0}+\varepsilon_{1}, \\
n\left(\varepsilon_{0}+\varepsilon_{1}\right) \leq c \varepsilon^{-\tau_{0}} \mathcal{N}_{0}^{\tau_{0}}+c \varepsilon^{-\tau_{1}} \mathcal{N}_{1}^{\tau_{1}},
\end{gathered}
$$

and consequently

$$
\mathbb{A}_{n}^{T C}(f)_{\infty} \leq c n^{-\alpha_{0}} \mathcal{N}_{0}+c n^{-\alpha_{1}} \mathcal{N}_{1}, \quad n=1,2, \ldots,
$$

with $c$ depending only on $\alpha_{0}, \alpha_{1}$, and the parameters of $\mathcal{T}$.

Proof. All the elements for the proof already appear in this subsection, especially in the proofs of Theorem 3.7 and Lemma 3.5, and we shall assume complete familiarity with the notation, terminology, and estimates given there. Denote the number of cells used in the "trim and cut" algorithm for $\left(b_{\theta}\right)$, with approximation error $\varepsilon$, by $n(\varepsilon)$. Similarly, let $n_{j}\left(\varepsilon_{j}\right)$ be the corresponding number of cells used for $f^{j}(j=0,1)$, again represented as $f^{j}=\sum_{\theta \in \Theta} b_{\theta}^{j} \varphi_{j}$, in place of Step 1. The theorem will be proved once we establish the estimate

$$
n\left(\varepsilon_{0}+\varepsilon_{1}\right) \leq 2\left(n_{0}\left(\varepsilon_{0}\right)+n_{1}\left(\varepsilon_{1}\right)\right)
$$

for any $\varepsilon_{0}, \varepsilon_{1}>0$. Indeed, by combining this inequality with the results of Theorem 3.7 (in particular, inequalities (3.16) $-(3.17)$ ), we can see that the estimate

$$
n\left(\varepsilon_{0}+\varepsilon_{1}\right) \leq 2 c \varepsilon_{0}^{-\tau_{0}} \mathcal{N}_{0}^{\tau_{0}}+2 c \varepsilon_{1}^{-\tau_{1}} \mathcal{N}_{1}^{\tau_{1}}=n
$$

is true if we set $\varepsilon_{j}:=(4 c)^{1 / \tau_{j}} n^{-1 / \tau_{j}} \mathcal{N}_{j}, j=0,1$, where $c$ is the constant appearing there. But the fact that $n \geq n\left(\varepsilon_{0}+\varepsilon_{1}\right)$ and the definition of $n(\cdot)$ imply

$$
\mathbb{A}_{n}^{T C}(f)_{\infty} \leq \mathbb{A}_{n\left(\varepsilon_{0}+\varepsilon_{1}\right)}^{T C}(f)_{\infty} \leq \varepsilon_{0}+\varepsilon_{1} .
$$

Hence, by the definition of the $\varepsilon_{j}$, the rightmost terms of this last inequality are bounded by the desired terms on the right-hand side of inequality (3.25).

In order to prove estimate (3.26), we only need to estimate the number of segments $\Sigma$ for $f$. First observe in Step 3 of the algorithm that for the thresholding condition (3.6) to hold for $f$, with $\varepsilon:=\varepsilon_{0}+\varepsilon_{1}$, the condition must also be satisfied for that same cell $\theta^{\diamond}$ for at least one of the $f^{j}$ with corresponding threshold $\varepsilon_{j}$ $(j=0,1)$. This shows that the tree $\Gamma^{\nu}=\Gamma^{\nu}(f, \varepsilon)$ determined by threshold $\varepsilon$ is contained in the union of the corresponding trees $\Gamma^{\nu}\left(f^{j}, \varepsilon_{j}\right)(j=0,1)$. By the construction of segments $\sigma$ from maximal chains of $\Gamma^{\nu}(f)$ in Step 4, the segments for $f$ are disjoint and one of the conditions (3.10)- (3.12) must hold. If (3.10) or (3.11) holds for a segment $\sigma$ of $f$, then $\sum_{\theta \in \sigma}\left|b_{\theta}^{0}+b_{\theta}^{1}\right|>\varepsilon_{0}+\varepsilon_{1}$ implies the corresponding condition for at least one of $f^{0}$ (and $\varepsilon_{0}$ ) or $f^{1}$ (and $\varepsilon_{1}$ ). That is, for one of $j=0,1$ we must have $\sum_{\theta \in \sigma}\left|b_{\theta}^{j}\right|>\varepsilon_{j}$, and so for at least half of the segments of $f$ this condition must persist for a fixed index $j(j=0,1)$. The number of remnant segments (see (3.12)), on the other hand, may be estimated by the sum of the number of remnant segments of $f^{0}$ and $f^{1}$, plus the number of new branching cells which may arise within the union of the trees of $f^{0}$ and $f^{1}$. These new cells are 
introduced in $\Gamma^{\nu}(f, \varepsilon)$ when two chains, exclusive to each of the $\Gamma^{\nu}\left(f^{j}, \varepsilon_{j}\right)$, meet, thereby dividing the existing chains for each of the trees and creating an additional segment. It is easy to see that the number of such new branching cells does not exceed $\min \left\{\Gamma_{f}^{\nu}\left(f^{0}, \varepsilon_{0}\right), \Gamma_{f}^{\nu}\left(f^{1}, \varepsilon_{1}\right)\right\}$.

This accounting of the three qualifying conditions (3.10)- 3.12 for segments gives

$$
\begin{aligned}
\left\lceil\frac{\# \Sigma}{2}\right\rceil & \leq \max \left\{\# \Sigma\left(f^{0}, \varepsilon_{0}\right), \# \Sigma\left(f^{1}, \varepsilon_{1}\right)\right\}+\min \left\{\Gamma_{f}^{\nu}\left(f^{0}, \varepsilon_{0}\right), \Gamma_{f}^{\nu}\left(f^{1}, \varepsilon_{1}\right)\right\} \\
& \leq \# \Sigma\left(f^{0}, \varepsilon_{0}\right)+\# \Sigma\left(f^{1}, \varepsilon_{1}\right),
\end{aligned}
$$

which implies the desired estimate $(\underline{3.26})$ and completes the proof.

3.2.2. The case $0<p<\infty$. We now return to completing Steps $3-5$ in the case that $p<\infty$. The arguments are quite similar to the case $p=\infty$ in the previous subsection, and we shall use the notation there and indicate only the differences. Introduce a new parameter $\varrho$, where $0<\varrho<p$, and fix $\varepsilon>0$.

Step 3. Trimming of $\Theta^{\nu}(1 \leq \nu \leq K)$ with $\varepsilon$. This step is the same as in Case 1 $(\overline{p=\infty)}$ with (3.5) replaced by

$$
\left(\sum_{\theta \subset \theta^{\diamond}}\left(\left|b_{\theta}\right||\theta|^{1 / p}\right)^{\varrho}\right)^{1 / \varrho} \leq \varepsilon .
$$

In contrast to the case $p=\infty$, the error $\left\|f_{\nu}-f_{\Gamma^{\nu}}\right\|_{p}$ is no longer controlled solely by $\varepsilon$. It will depend on the smoothness of the function $f$ that is being approximated (see Theorem 3.9 below).

Step 4. Partitioning the branches of each tree $\Gamma^{\nu}$ into chains and the chains into "segments". We proceed exactly as in the case $p=\infty$, replacing conditions (3.10)(3.12) by the following:

$$
\left|b_{\theta_{i}}\right|\left|\theta_{i}\right|^{1 / p}>\varepsilon \quad(\text { case of } \mu=0),
$$

$$
\begin{aligned}
&\left(\sum_{j=i}^{i+\mu-1}\left(\left|b_{\theta_{j}}\right|\left|\theta_{j}\right|^{1 / p}\right)^{\varrho}\right)^{1 / \varrho} \leq \varepsilon, \text { but }\left(\sum_{j=i}^{i+\mu}\left(\left|b_{\theta_{j}}\right|\left|\theta_{j}\right|^{1 / p}\right)^{\varrho}\right)^{1 / \varrho}>\varepsilon(\text { case of } \mu>0), \\
&\left(\sum_{j=i}^{l}\left(\left|b_{\theta_{j}}\right|\left|\theta_{j}\right|^{1 / p}\right)^{\varrho}\right)^{1 / \varrho} \leq \varepsilon .
\end{aligned}
$$

Step 5. Rewriting elements from certain segments of $\Sigma^{\nu}$. This step is exactly the $\overline{\text { same as }}$ for the case $p=\infty$.

- Error estimation for the "trim and cut" algorithm (case $0<p<\infty)$.

Suppose that the "trim and cut" algorithm has been applied to a function $f$ with $0<\varrho<p$ and $\varepsilon>0$, as described above. Let $A_{\varepsilon}^{T C}(f)_{p}=\sum_{\theta \in \Lambda_{\varepsilon}} c_{\theta} \varphi_{\theta}, \Lambda_{\varepsilon} \subset \Theta$, be the approximant produced by the algorithm. We denote

$$
n(\varepsilon):=\# \Lambda_{\varepsilon}, \quad \mathbb{A}_{n(\varepsilon)}^{T C}(f)_{p}:=\left\|f-A_{\varepsilon}^{T C}(f)_{p}\right\|_{p},
$$


and

$$
\mathbb{A}_{n}^{T C}(f)_{p}:=\inf \left\{\mathbb{A}_{n(\varepsilon)}^{T C}(f)_{p}: n(\varepsilon) \leq n\right\} .
$$

Theorem 3.9. If $f \in B_{\tau}^{\alpha}(\mathcal{T})$, where $\alpha \geq 1 / \varrho-1 / p$ and $\tau=\left(\alpha+\frac{1}{p}\right)^{-1}$, then for each $\varepsilon>0$,

$$
\mathbb{A}_{n(\varepsilon)}^{T C}(f)_{p} \leq c \varepsilon^{\alpha \tau}\|f\|_{B_{\tau}^{\alpha}(\mathcal{T})}^{\tau / p} \quad \text { and } \quad n(\varepsilon) \leq c \varepsilon^{-\tau}\|f\|_{B_{\tau}^{\alpha}(\mathcal{T})}^{\tau},
$$

and hence

$$
\mathbb{A}_{n}^{T C}(f)_{p} \leq c n^{-\alpha}\|f\|_{B_{\tau}^{\alpha}(\mathcal{T})}, \quad n=1,2, \ldots,
$$

where $c$ depends on $p, \varrho, \alpha$, and the parameters of $\mathcal{T}$.

Proof. We first estimate $n(\varepsilon)$. From the stopping time criterium (the converse inequality of (3.28) ) in Step 3, it follows that

$$
\varepsilon<\left(\sum_{\theta \subset \theta^{\diamond}}\left(\left|b_{\theta}\right||\theta|^{1 / p}\right)^{\varrho}\right)^{1 / \varrho} \leq\left(\sum_{\theta \subset \theta^{\diamond}}\left(\left|b_{\theta}\right||\theta|^{1 / p}\right)^{\tau}\right)^{1 / \tau} \quad(\text { since } \tau \leq \varrho)
$$

for each $\theta^{\diamond} \in \Gamma_{f}^{\nu}$, which enables us to repeat the arguments from the proof of Theorem 3.7 and obtain the estimate $\# \Gamma_{f}^{\nu} \leq c \varepsilon^{-\tau}\|f\|_{B_{\tau}^{\alpha}(\mathcal{T})}^{\tau}$. In going further, we use (3.30) in a similar fashion and the above to infer as in the proof of Theorem 3.7 that

$$
\# \Sigma^{\nu} \leq c \varepsilon^{-\tau}\|f\|_{B_{\tau}^{\alpha}(\mathcal{T})}^{\tau} .
$$

This implies the desired estimate for $n(\varepsilon)$.

It remains to estimate the error $\left\|f-A_{n(\varepsilon)}^{T C}(f)_{p}\right\|_{p}$. We first estimate $\left\|f_{\nu}-f_{\Gamma^{\nu}}\right\|_{p}$. To this end, we group the removed cells into collections of comparable $B_{\tau}^{\alpha}$-norms. We denote by

$$
\Xi^{\nu}:=\left\{\theta \in \Theta^{\nu} \backslash \Gamma^{\nu}: \theta \nsubseteq \theta^{\prime} \text { for any } \theta^{\prime} \in \Theta^{\nu} \backslash \Gamma^{\nu}, \theta^{\prime} \neq \theta\right\}
$$

the set of all cells at which a trimmed branch starts. Note that for each $\theta^{\diamond} \in \Xi^{\nu}$ the inequality (3.28) holds. Therefore, we can partition $\Xi^{\nu}$ into disjoint collections $\Xi_{j}^{\nu}, j=1,2, \ldots, L^{\nu}$, such that $\Xi^{\nu}=\bigcup_{j=1}^{L^{\nu}} \Xi_{j}^{\nu}$ and

$$
\varepsilon^{\varrho}<\sum_{\theta^{\curvearrowright} \in \Xi_{j}^{\nu}} \sum_{\theta \subset \theta^{\diamond}}\left(\left|b_{\theta} \| \theta\right|^{1 / p}\right)^{\varrho} \leq 2 \varepsilon^{\varrho}
$$

for all $j=1,2, \ldots, L^{\nu}$ except possibly for $j=L^{\nu}$, when the leftmost inequality may fail. Hence, since the cells from $\Xi^{\nu}$ have disjoint interiors, and recalling that $\left|b_{\theta}\left\|\left.\theta\right|^{1 / p} \approx\right\| b_{\theta} \varphi_{\theta} \|_{p}\right.$, we obtain

$$
\begin{aligned}
\left\|f_{\nu}-f_{\Gamma^{\nu}}\right\|_{p} & =\left\|\sum_{j=1}^{L^{\nu}} \sum_{\theta^{\diamond} \in \Xi_{j}^{\nu}} \sum_{\theta \subset \theta^{\diamond}} b_{\theta} \varphi_{\theta}\right\|_{p} \leq\left(\sum_{j=1}^{L^{\nu}}\left\|\sum_{\theta^{\diamond} \in \Xi_{j}^{\nu}} \sum_{\theta \subset \theta^{\diamond}} b_{\theta} \varphi_{\theta}\right\|_{p}^{p}\right)^{\frac{1}{p}} \\
& \leq c\left(\sum_{j=1}^{L^{\nu}}\left[\sum_{\theta^{\diamond} \in \Xi_{j}^{\nu}} \sum_{\theta \subset \theta^{\diamond}}\left(\left|b_{\theta} \| \theta\right|^{1 / p}\right)^{\varrho}\right]^{p / \varrho}\right)^{\frac{1}{p}} \\
& \leq c\left(\sum_{j=1}^{L^{\nu}} 2^{p / \varrho} \varepsilon^{p}\right)^{1 / p}=c\left(L^{\nu}\right)^{1 / p} \varepsilon
\end{aligned}
$$


where we used the embedding inequality (2.26). To estimate $L^{\nu}$ we once again exploit the idea used in estimating $\# \Gamma_{f}^{\nu}($ see (3.18) $-(3.19)$ ). Since $0<\tau \leq \varrho$, we have by (3.36) that

$$
\varepsilon<\left(\sum_{\theta^{\diamond} \in \Xi_{j}^{\nu}} \sum_{\theta \subset \theta^{\diamond}}\left(\left|b_{\theta}\right||\theta|^{1 / p}\right)^{\varrho}\right)^{1 / \varrho} \leq\left(\sum_{\theta^{\diamond} \in \Xi_{j}^{\nu}} \sum_{\theta \subset \theta^{\diamond}}\left(\left|b_{\theta}\right||\theta|^{1 / p}\right)^{\tau}\right)^{1 / \tau} .
$$

We use this and the fact that the collections $\Xi_{j}^{\nu}$ are disjoint to obtain

$$
L^{\nu} \cdot \varepsilon^{\tau} \leq c \sum_{j=1}^{L^{\nu}}\left(\sum_{\theta^{\diamond} \in \Xi_{j}^{\nu}} \sum_{\theta \subset \theta^{\diamond}}\left\|b_{\theta} \varphi_{\theta}\right\|_{p}^{\tau}\right) \leq c\left\|f_{\nu}\right\|_{B_{\tau}^{\alpha}(\mathcal{T})}^{\tau} .
$$

Combining (3.37) and (3.38), we obtain

$$
\left\|f_{\nu}-f_{\Gamma^{\nu}}\right\|_{p} \leq c\left(\varepsilon^{-\tau}\left\|f_{\nu}\right\|_{B_{\tau}^{\alpha}(\mathcal{T})}^{\tau}\right)^{1 / p} \varepsilon=c \varepsilon^{\alpha \tau}\left\|f_{\nu}\right\|_{B_{\tau}^{\alpha}(\mathcal{T})}^{\tau / p},
$$

and hence by standard subaddivitity estimates for $L_{p}(0<p<\infty)$ we may estimate the sum

$$
\begin{aligned}
\left\|f-f_{\Gamma}\right\|_{p} & \leq\left(\sum_{\nu=1}^{K}\left\|f_{\nu}-f_{\Gamma^{\nu}}\right\|_{p}^{p^{*}}\right)^{1 / p^{*}} \\
& \leq c \varepsilon^{\alpha \tau}\left(\sum_{\nu=1}^{K}\left(\left\|f_{\nu}\right\|_{B_{\tau}^{\alpha}(\mathcal{T})}^{\tau}\right)^{p^{*} / p}\right)^{1 / p^{*}} \leq c \varepsilon^{\alpha \tau}\|f\|_{B_{\tau}^{\alpha}(\mathcal{T})}^{\tau / p},
\end{aligned}
$$

where $p^{*}:=\min \{1, p\}$.

To complete the proof of the theorem, we must estimate $\left\|f_{\Gamma^{\nu}}-A^{\nu}\right\|_{p}$. This differs from our earlier arguments in the case $p=\infty$, which involved the error estimate (3.15) over a ring of a segment. For any such ring $R_{\sigma}\left(\sigma \in \Sigma^{\nu}\right)$ we use instead the estimate

$$
\begin{aligned}
\left\|f_{\Gamma^{\nu}}-A^{\nu}\right\|_{L_{p}\left(R_{\sigma}\right)} & =\left\|\sum_{\theta \in \sigma} b_{\theta} \varphi_{\theta}-\sum_{\theta \in \mathcal{X}_{\sigma}} c_{\theta} \varphi_{\theta}\right\|_{L_{p}\left(R_{\sigma}\right)} \leq\left\|\sum_{\theta \in \sigma^{\prime}} b_{\theta} \varphi_{\theta}\right\|_{L_{p}\left(\theta_{1}\right)} \\
& \leq c\left(\sum_{\theta \in \sigma^{\prime}}\left\|b_{\theta} \varphi_{\theta}\right\|_{p}^{\tau}\right)^{1 / \tau} \leq c\left(\sum_{\theta \in \sigma^{\prime}}\left(\left|b_{\theta} \| \theta\right|^{1 / p}\right)^{\varrho}\right)^{1 / \varrho} \leq c \varepsilon
\end{aligned}
$$

where we used the embedding inequality (2.26). From the above, using that all rings $\left\{R_{\sigma}\right\}_{\sigma \in \Sigma^{\nu}}$ have disjoint interiors, we obtain

$$
\left\|f_{\Gamma^{\nu}}-A^{\nu}\right\|_{p} \leq\left(\sum_{\sigma \in \Sigma^{\nu}}\left\|f_{\Gamma^{\nu}}-A^{\nu}\right\|_{L_{p}\left(R_{\sigma}\right)}^{p}\right)^{1 / p} \leq c\left(\# \Sigma^{\nu}\right)^{1 / p} \varepsilon
$$

Combining (3.40) and (3.35) yields

$$
\left\|f_{\Gamma^{\nu}}-A^{\nu}\right\|_{p} \leq c \varepsilon^{\alpha \tau}\left\|f_{\nu}\right\|_{B_{\tau}^{\alpha}(\mathcal{T})}^{\tau / p}
$$

and hence

$$
\begin{aligned}
\left\|f_{\Gamma}-A_{\varepsilon}^{T C}(f)_{p}\right\|_{p} & \leq\left(\sum_{\nu=1}^{K}\left\|f_{\Gamma^{\nu}}-A^{\nu}\right\|_{p}^{p^{*}}\right)^{1 / p^{*}} \\
& \leq c \varepsilon^{\alpha \tau}\left(\sum_{\nu=1}^{K}\left(\left\|f_{\nu}\right\|_{B_{\tau}^{\alpha}(\mathcal{T})}^{\tau}\right)^{p^{*} / p}\right)^{1 / p^{*}} \leq c \varepsilon^{\alpha \tau}\|f\|_{B_{\tau}^{\alpha}(\mathcal{T})}^{\tau / p}
\end{aligned}
$$


where $p^{*}:=\min \{1, p\}$. From this and (3.39), we obtain the appropriate estimate which corresponds to (3.14) of the case for $p=\infty$ :

$$
\left\|f-A_{\varepsilon}^{T C}(f)_{p}\right\|_{p} \leq c \varepsilon^{\alpha \tau}\|f\|_{B_{\tau}^{\alpha}(\mathcal{T})}^{\tau / p} .
$$

Lemma 3.10. Let $f=f^{0}+f^{1}$, where $f=\sum_{\theta \in \Theta} b_{\theta} \varphi_{\theta}, f^{j}=\sum_{\theta \in \Theta} b_{\theta}^{j} \varphi_{\theta}(j=0,1)$ with $b_{\theta}=b_{\theta}^{1}+b_{\theta}^{2}($ all $\theta)$, and let

$$
\mathcal{N}_{j}:=\left(\sum_{\theta \in \Theta}\left(\left|b_{\theta}^{j}\right||\theta|^{1 / p}\right)^{\tau_{j}}\right)^{1 / \tau_{j}}<\infty \quad(j=0,1)
$$

with $\alpha_{j} \geq \frac{1}{\varrho}-\frac{1}{p}(0<\varrho<p)$ and $\tau_{j}:=1 /\left(\alpha_{j}+\frac{1}{p}\right)^{-1}, j=0,1$. Furthermore, suppose the "trim and cut" algorithm has been applied to $f$, using the above representation of $f$ in place of Step 1 , with $0<\varrho<p$ as above and $\varepsilon=\varepsilon_{0}+\varepsilon_{1}$ for some $\varepsilon_{0}, \varepsilon_{1}>0$. Then we have

$$
\begin{aligned}
\mathbb{A}_{n\left(\varepsilon_{0}+\varepsilon_{1}\right)}^{T C}(f)_{p} & \leq c\left(\varepsilon_{0}+\varepsilon_{1}\right)\left(\varepsilon_{0}^{-\tau_{0}} \mathcal{N}_{0}^{\tau_{0}}+\varepsilon_{1}^{-\tau_{1}} \mathcal{N}_{1}^{\tau_{1}}\right)^{1 / p}, \\
n\left(\varepsilon_{0}+\varepsilon_{1}\right) & \leq c\left(\varepsilon_{0}^{-\tau_{0}} \mathcal{N}_{0}^{\tau_{0}}+\varepsilon_{1}^{-\tau_{1}} \mathcal{N}_{1}^{\tau_{1}}\right),
\end{aligned}
$$

and, therefore,

$$
\mathbb{A}_{n}^{T C}(f)_{p} \leq c\left(n^{-\alpha_{0}} \mathcal{N}_{0}+n^{-\alpha_{1}} \mathcal{N}_{1}\right), \quad n=1,2, \ldots,
$$

where $c$ depends only on $p, \varrho, \alpha_{0}, \alpha_{1}$, and the parameters of $\mathcal{T}$.

Proof. The proof is very similar to the proof of Theorem 3.9 and we shall only indicate the differences, using the notation and ideas from there. Those differences are in estimating $\# \Gamma_{f}^{\nu}, \# \Sigma^{\nu}$ and $L^{\nu}$ (see (3.35) and (3.38)). From the stopping criterium (converse inequality to (3.28)) in Step 3, it follows that, for $\theta^{\diamond} \in \Gamma_{f}^{\nu}$,

$$
\begin{aligned}
\varepsilon_{0}+\varepsilon_{1} & <\left(\sum_{\theta \subset \theta^{\diamond}}\left(\left|b_{\theta}\right||\theta|^{1 / p}\right)^{\varrho}\right)^{1 / \varrho} \\
& \leq c_{\varrho}\left(\sum_{\theta \subset \theta^{\diamond}}\left(\left|b_{\theta}^{0} \| \theta\right|^{1 / p}\right)^{\varrho}\right)^{1 / \varrho}+c_{\varrho}\left(\sum_{\theta \subset \theta^{\diamond}}\left(\left|b_{\theta}^{1}\right||\theta|^{1 / p}\right)^{\varrho}\right)^{1 / \varrho} \\
& \leq c_{\varrho}\left(\sum_{\theta \subset \theta^{\diamond}}\left(\left|b_{\theta}^{0} \| \theta\right|^{1 / p}\right)^{\tau_{0}}\right)^{1 / \tau_{0}}+c_{\varrho}\left(\sum_{\theta \subset \theta^{\diamond}}\left(\left|b_{\theta}^{1}\right||\theta|^{1 / p}\right)^{\tau_{1}}\right)^{1 / \tau_{1}}
\end{aligned}
$$

where $c_{\varrho}:=\max \left\{1,2^{1 / \varrho-1}\right\}$ and we used the fact that $\tau_{0}, \tau_{1} \leq \varrho$. Therefore, for each $\theta^{\diamond} \in \Gamma_{f}^{\nu}$, at least one of

$$
\varepsilon_{0}<c_{\varrho}\left(\sum_{\theta \subset \theta^{\diamond}}\left(\left|b_{\theta}^{0}\right||\theta|^{1 / p}\right)^{\tau_{0}}\right)^{1 / \tau_{0}} \text { or } \varepsilon_{1}<c_{\varrho}\left(\sum_{\theta \subset \theta^{\diamond}}\left(\left|b_{\theta}^{1}\right||\theta|^{1 / p}\right)^{\tau_{1}}\right)^{1 / \tau_{1}}
$$

must hold. Denoting by $\Gamma_{f^{0}}^{\nu}$ and $\Gamma_{f^{1}}^{\nu}$ the sets of all $\theta^{\diamond} \in \Gamma_{f}^{\nu}$ for which the first or second inequality, respectively, holds, we obtain

$$
\mathcal{N}_{j}^{\tau_{j}} \geq c \sum_{\theta^{\diamond} \in \Gamma_{f^{j}}^{\nu}} \sum_{\theta \subset \theta^{\diamond}}\left(\left|b_{\theta}^{j} \| \theta\right|^{1 / p}\right)^{\tau_{j}} \geq c \# \Gamma_{f^{j}}^{\nu} \varepsilon_{j}^{\tau_{j}}(j=0,1)
$$


and hence

$$
\# \Gamma_{f}^{\nu} \leq \# \Gamma_{f^{0}}^{\nu}+\# \Gamma_{f^{1}}^{\nu} \leq c\left(\varepsilon_{0}^{-\tau_{0}} \mathcal{N}_{0}^{\tau_{0}}+\varepsilon_{1}^{-\tau_{1}} \mathcal{N}_{1}^{\tau_{1}}\right)
$$

We obtain similar (with the same right-hand-side quantity) for $\# \Sigma^{\nu}$ and $L^{\nu}$ by using the same argument. The estimate for $\# \Sigma^{\nu}$ gives the desired estimate for $n\left(\varepsilon_{0}+\varepsilon_{1}\right)$.

We may use estimates (3.37) and (3.40) in the proof of Theorem 3.9, with $\varepsilon=$ $\varepsilon_{0}+\varepsilon_{1}$, together with the above estimates for $\# \Sigma^{\nu}$ and $L^{\nu}$, to obtain

$$
\mathbb{A}_{n\left(\varepsilon_{0}+\varepsilon_{1}\right)}^{T C}(f)_{p} \leq c\left(\varepsilon_{0}+\varepsilon_{1}\right)(\# \Sigma)^{1 / p},
$$

from which the desired estimate (3.42) follows. The final estimate (3.44) is proved by selecting $\varepsilon_{j}=(2 c / n)^{1 / \tau_{j}} \mathcal{N}_{j}$, which by our result (3.43), gives that $n\left(\varepsilon_{0}+\varepsilon_{1}\right) \leq n$ and so

$$
\mathbb{A}_{n}^{T C}(f)_{p} \leq \mathbb{A}_{n\left(\varepsilon_{0}+\varepsilon_{1}\right)}^{T C}(f)_{p} \leq c n^{1 / p}\left(\varepsilon_{0}+\varepsilon_{1}\right) \leq c\left(n^{-\alpha_{0}} \mathcal{N}_{0}+n^{-\alpha_{1}} \mathcal{N}_{1}\right),
$$

where we have used (3.46) in the second inequality.

3.3. "Push the error" algorithm. The idea of this algorithm to our knowledge first appeared in [5]. Our goal is to adapt this algorithm for nonlinear $n$-term Courant element approximation in the uniform norm and perfect it so that the resulting algorithm achieves the rate of convergence of the best approximation.

In 3.3.1 we describe the "push the error" algorithm in its simplest and most naive form. We follow with three examples which illustrate deficiencies of the simple algorithm and the types of traps to which it may fall prey. In $\S 3.3 .2$, we give our refined version of that algorithm. Throughout this section, we assume that $\mathcal{T}=\bigcup_{m=0}^{\infty} \mathcal{T}_{m}$ is an LR-triangulation of some compact polygonal domain $E$ in $\mathbb{R}^{2}$, where the approximation takes place (see $\S 2.1$ ), and $f \in C(E)$.

3.3.1. A naive "push the error" algorithm $(p=\infty)$. We begin by outlining the basic elements of the algorithm.

Step 1 (Decompose). In this subsection we denote by $Q_{j}(f)$ the piecewise linear continuous function that interpolates $f$ at the vertices $V_{j}$ of all triangles from $\mathcal{T}_{j}$. Clearly $f \in C(E)$ can be represented as follows:

$$
f=Q_{0}(f)+\sum_{j=1}^{\infty}\left(Q_{j}(f)-Q_{j-1}(f)\right)=: \sum_{\theta \in \Theta} c_{\theta} \varphi_{\theta},
$$

where the series converges uniformly. In practice the series terminates at some finest level $\Theta_{J}(J>1)$, so that

$$
f=\sum_{j=0}^{J} \sum_{\theta \in \Theta_{j}} c_{\theta} \varphi_{\theta}
$$

Assuming that initially $f=\sum_{\theta \in \Theta_{J}} c_{\theta} \varphi_{\theta}$, there exists a fast and efficient procedure for obtaining (3.47).

Step 2 ("Threshold" and "push the error"). Fix $\varepsilon>0$. We shall begin at the coarsest level $\Theta_{0}$ and proceed consecutively through to higher resolution levels 
$\Theta_{1}, \Theta_{2}, \ldots, \Theta_{J}$. We define $\Lambda_{0}$ as the set of all cells $\theta \in \Theta_{0}$ such that $\left|c_{\theta}\right|>\varepsilon$ $\left(\left\|\varphi_{\theta}\right\|=1\right)$, and set

$$
A_{0}:=\sum_{\theta \in \Lambda_{0}} c_{\theta} \varphi_{\theta}=: \sum_{\theta \in \Theta_{0}} b_{\theta} \varphi_{\theta}
$$

Next we rewrite all remaining terms $c_{\theta} \varphi_{\theta}\left(\theta \in \Theta_{0} \backslash \Lambda_{0}\right)$ at the next finer level and add the resulting terms to the corresponding terms from $\left(c_{\theta} \varphi_{\theta}\right)_{\theta \in \Theta_{1}}$. Thus we obtain a representation of $f$ in the form

$$
f=A_{0}+\sum_{\theta \in \Theta_{1}} b_{\theta} \varphi_{\theta}+\sum_{j=2}^{J} \sum_{\theta \in \Theta_{j}} c_{\theta} \varphi_{\theta} .
$$

We next process the Courant elements at level $\Theta_{1}$. We define $\Lambda_{1}$ as the set of all $\theta \in \Theta_{1}$ such that $\left|b_{\theta}\right|>\varepsilon$, and set $A_{1}:=\sum_{\theta \in \Lambda_{1}} b_{\theta} \varphi_{\theta}$. All remaining terms $b_{\theta} \varphi_{\theta}$, $\theta \in \Theta_{1} \backslash \Lambda_{1}$, we rewrite at the finer level $\Theta_{2}$ and add the resulting terms to the corresponding terms $\left(c_{\theta} \varphi_{\theta}\right)_{\theta \in \Theta_{2}}$. The representation of $f$ at this stage is written as

$$
f=A_{0}+A_{1}+\sum_{\theta \in \Theta_{2}} b_{\theta} \varphi_{\theta}+\sum_{j=3}^{J} \sum_{\theta \in \Theta_{j}} c_{\theta} \varphi_{\theta} .
$$

We continue in this way until we reach the finest (i.e., highest resolution) level $\Theta_{J}$. The only modification at this finest level is that we discard all terms whose coefficients in absolute value do not exceed our threshold parameter $\varepsilon$. In this way we obtain our approximation

$$
A:=A_{\varepsilon}(f):=\sum_{j=0}^{J} A_{j}=\sum_{\theta \in \Lambda} b_{\theta} \varphi_{\theta}, \quad \Lambda:=\bigcup_{j=0}^{J} \Lambda_{j} .
$$

Since only small terms $\left(\left|b_{\theta}\right| \leq \varepsilon\right)$ at a single (in this case, finest) level are discarded, they cannot stack up, and we have

$$
\left\|f-A_{\varepsilon}(f)\right\|_{\infty} \leq \varepsilon .
$$

Some modifications must be made, however, to insure that this simple and efficient algorithm will achieve sparse representations in an asymptotically optimal sense and avoid hidden traps that will result in using too many terms in the approximation.

We indicate briefly each of the possible pitfalls to keep in mind, before developing the algorithm in full in the next subsection.

Trap 1. The interpolation scheme we used to represent $f$ in (3.47) leads to difficulties, since it does not always lead to sparse representations. We give here a univariate example which may be easily extended to two dimensions.

Let $E:=[-1,1]$, and let $f$ be the hat function on $\left[-\frac{1}{2^{N}}, \frac{1}{2^{N}}\right]$ for $N$ sufficiently large, i.e., $f(x)=\varphi\left(2^{N} x\right)$ with $\varphi(x):=(1-|x|) \mathbb{1}_{[-1,1]}(x), x \in \mathbb{R}$. We assume that $\mathcal{T}$ consists of all dyadic subintervals of $[-1,1]$. Using the interpolation scheme described in Step 1 at the coarsest level, we must interpolate the extremes at $-1,0,1$ in order to decrease the $L^{\infty}$ error. The resulting error after this stage, however, is $1-\frac{1}{2^{N}}$. Proceeding with the naive "push-the-error" algorithm with any $\varepsilon<\frac{1}{2}$ results in an index set $\Lambda$ with $\# \Lambda \sim N$. However, the best approximation is achieved using the single fine scale element $\varphi\left(2^{N} x\right)$. Therefore, any reasonable algorithm that retains $n$ terms in the approximant should give a rate of convergence $\mathcal{O}\left(n^{-\gamma}\right)$ for any $\gamma>0$. 
Trap 2. For a given $\varepsilon>0$ the algorithm as currently described may produce a great number of undesired terms due to the superposition of a large number of fine level nonintersecting terms $\left(c_{\theta} \varphi_{\theta}\right)$ with a single coarse level term $\left(\varphi_{\theta_{0}}\right)$ :

$$
f=\varepsilon\left(\varphi_{[-1,1]}+\sum_{\theta \in \mathcal{M}} \delta \varphi_{\theta}\right) .
$$

We set $\mathcal{M}$ as a set of disjoint cells $\theta$ from level $2^{2 N}$ with $\theta \subset(-\delta, \delta)$, where $\delta=2^{-N}$. It is clear that we can choose these cells for $\mathcal{M}$ so that $\# \mathcal{M}=2^{N}$. At the central vertex $x_{\theta}$ of each cell $\theta$ we have $f\left(x_{\theta}\right)>\varepsilon(1-\delta)+\delta \varepsilon=\varepsilon$. The "push-the-error algorithm" will produce an inefficient approximation, since it will not select the coarse first term in (3.49) as one might hope. Instead, no such element will be chosen at the coarsest level, and the error will be pushed. At each successive stage the coefficients of the rewritten descendant Courant elements for $\theta_{0}$ will all again lie beneath the threshold and be further rewritten until all cells are on level $2^{2 N}$. At that stage they will be combined with the remaining terms in (3.49). The corresponding cells will now have coefficients that exceed the threshold and must be selected, producing at least $2^{N}$ terms in the approximant. As indicated above, a desirable algorithm should have anticipated the trap of many small, finely supported elements that may come at a late stage, and would have chosen for this function the approximation (with threshold $\varepsilon$ ) that consists of a single element, namely $\varepsilon \varphi_{[-1,1]}$.

Trap 3. The final example is one that outmaneuvers a quick remedy to Trap 2, i.e., merely thresholding all small terms at the finest level. For a given $\varepsilon>0$, we define

$$
f=\varepsilon \varphi_{[-1,1]}+\sum_{j=1}^{N} \delta_{j} \varphi_{\left[0,2^{-m_{j}}\right]}+\varepsilon \varphi_{\left[0,2^{-M}\right]},
$$

where $m_{j}=j^{2}, \delta_{j}=2^{-j} \varepsilon$, and $M=2^{N^{2}}$. In this example, elements are again building near the origin, but now appear at many levels with small amplitudes. The "push-the-error" algorithm will again take no elements at the coarsest level and push the error to the next level. Continuing with the algorithm, we are forced to take essentially all terms as the approximation to the given function when, optimally, only two terms need be taken.

It is obvious that we can take each of these template examples as building blocks and build functions to cause these problems for all $\varepsilon$, at all locations and scales.

3.3.2. "Push the error" algorithm in the uniform norm $(p=\infty)$. In this section we indicate the refinements needed in order to guarantee that the "push the error" algorithm will achieve optimal rates of approximation. As with the "trim and cut" algorithm, we break it down into manageable steps.

\section{- Description of the algorithm.}

Step 1 (Decompose). For $f \in C(E)$ initially represented by (3.1), we may assume, without loss of generality, that there exists a finest level $\Theta_{J}(J>0)$ such that $f$ is written as

$$
f=\sum_{j=0}^{J} \sum_{\theta \in \Theta_{j}} b_{\theta} \varphi_{\theta}
$$


Step 2 ("Prune the shrubs"). In the current algorithm we are not able to organize the cells of $\Theta$ into trees as we did in the "trim and cut" method, since, once we rewrite the error on a finer level, adjacent trees are immediately affected and we lose the benefit of their intended organization properties. This step of our algorithm, however, is analogous to Step 3 of the "trim and cut" algorithm. We fix $\varepsilon>0$ and let $\varepsilon^{*}:=\varepsilon / 2$. Our goal is, by discarding small insignificant terms $b_{\theta} \varphi_{\theta}$ in the representation of $f$ from $(3.50)$, to prevent our refined algorithm from being trapped by a situation such as that described in "Trap 2" (see the naive "push the error" algorithm of §3.3.1). We shall remove such terms, but insure that the resulting uniform error is at most $\varepsilon^{*}$ and denote by $\Gamma$ the set of all retained cells. In addition, we shall construct a set $\Gamma_{f} \subset \Gamma$, consisting of "final cells" in $\Gamma$.

First, we need to introduce an organizational concept as a replacement for the tree structures of $\S 3.2$. We shall say (figuratively) that a cell $\theta \in \Theta$ sits on another cell $\theta^{\diamond} \in \Theta$, if $\theta$ is at least as fine as $\theta^{\diamond}$ and its interior (denoted by $\theta^{\circ}$ ) intersects the interior of $\theta^{\diamond}$. Furthermore, for $\theta^{\diamond} \in \Theta$, we denote the collection of all cells that sit on $\theta^{\diamond}$ by

$$
\mathcal{Y}_{\theta^{\diamond}}:=\left\{\theta \in \Theta: \theta^{\circ} \cap \theta^{\diamond} \neq \emptyset \text { and } \operatorname{level}(\theta) \geq \operatorname{level}\left(\theta^{\diamond}\right)\right\} .
$$

The procedure of Step 2 will begin at the finest level and proceed to the coarsest, level by level, constructing sets $\Gamma_{f}$ and $\Gamma$. To initialize the procedure we put into $\Gamma_{f}$ all significant cells $\theta \in \Theta_{J}$, i.e., such that $\left|b_{\theta}\right|>\varepsilon^{*}$. We place in $\Gamma$ any cell from $\Theta_{J}$ that sits on a cell from $\Gamma_{f}$.

The inductive step proceeds as follows. Suppose that all cells from $\Theta_{j}$ with levels $j>m(0 \leq m<J)$ have already been processed. We now describe how to process $\Theta_{m}$. We place into $\Gamma_{f}$ all cells $\theta^{\diamond} \in \Theta_{m}$ that satisfy

$$
\sum_{\theta \in \mathcal{Y}_{\theta \diamond}}\left|b_{\theta}\right|>\varepsilon^{*}
$$

and for which there is no $\theta \in \Gamma_{f}$ from a higher level (i.e., $>m$ ) that sits on $\theta^{\diamond}$. A cell $\theta^{\diamond}$ from $\Theta_{m}$ is placed in $\Gamma$ if there is a cell $\theta$ in the current $\Gamma_{f}$ that sits on $\theta^{\diamond}$. We may consider the current version of $\Gamma_{f}$ as an intermediate ( $m$-th) version of a final set for $\Gamma$. Obviously, a cell $\theta^{\diamond}$ from $\Theta_{m}$ is discarded and not placed in $\Gamma$ if

$$
\sum_{\theta \in \mathcal{Y}_{\theta^{\diamond}}}\left|b_{\theta}\right| \leq \varepsilon^{*}
$$

and there is $\underline{\text { no }} \theta \in \Gamma_{f}$ from level $m$ or finer that sits on $\theta^{\diamond}$.

The procedure is terminated after $\Theta_{0}$ is processed and Step 2 of the algorithm is completed.

The two sets of cells $\Gamma$ and $\Gamma_{f}\left(\Gamma_{f} \subset \Gamma \subset \Theta\right)$ produced by Step 2 have the following properties, which follow directly from their construction:

(i) if $\theta_{1}, \theta_{2} \in \Gamma_{f}$ and level $\left(\theta_{1}\right) \neq \operatorname{level}\left(\theta_{2}\right)$, then $\theta_{1}^{\circ} \cap \theta_{2}^{\circ}=\emptyset$;

(ii) for each $\theta^{\diamond} \in \Gamma_{f}$, the inequality (3.52) holds;

(iii) for each $\theta^{\diamond} \in \Gamma$, there exists $\theta \in \Gamma_{f}$ that sits on $\theta^{\diamond}$.

We set $f_{\Gamma}:=\sum_{\theta \in \Gamma} b_{\theta} \varphi_{\theta}$ and define

$$
a_{\theta}:= \begin{cases}b_{\theta}, & \text { if } \theta \in \Gamma \\ 0, & \text { if } \theta \in \Theta \backslash \Gamma\end{cases}
$$


then obviously

$$
f_{\Gamma}=\sum_{\theta \in \Theta} a_{\theta} \varphi_{\theta}
$$

It follows from the construction that

$$
\left\|f-f_{\Gamma}\right\|_{\infty} \leq \varepsilon^{*} .
$$

Indeed, to see that this estimate holds, we let $\mathcal{D}$ denote the set of all cells $\theta \in \Theta$ that were discarded during the implementation of Step 2, i.e., $\mathcal{D}=\Theta \backslash \Gamma$. Let $x \in E$ be arbitrary. If $x \notin \bigcup_{\theta \in \mathcal{D}} \theta$, then $x$ does not belong to any cell that was discarded, and so $f_{\Gamma}(x)=f(x)$. On the other hand, if $x \in \bigcup_{\theta \in \mathcal{D}} \theta$, then there exists a cell $\theta^{\diamond} \in \mathcal{D}$ that contains $x$ and has coarsest level. Since $\theta^{\diamond}$ was discarded, the inequality (3.53) must hold. It follows that

$$
\left|f(x)-f_{\Gamma}(x)\right|=\left|\sum_{\theta \in \mathcal{D}} b_{\theta} \varphi_{\theta}(x)\right| \leq \sum_{\theta \in \mathcal{Y}_{\theta}}\left|b_{\theta}\right| \leq \varepsilon^{*},
$$

where we have normalized our elements so that $\left\|\varphi_{\theta}\right\|_{\infty}=1$. This verifies the desired inequality (3.56).

Step 3 (Push the error). We now process cells of $f_{\Gamma}$ with $\varepsilon^{*}$, starting from the coarsest level $\Theta_{0}$ and continuing to finer levels. The outcome of this step will be an approximant $\mathcal{A}:=\mathcal{A}_{\varepsilon}^{P}(f)$ of the form

$$
\mathcal{A}=\sum_{j=0}^{J} \mathcal{A}_{j}:=\sum_{j=0}^{J} \sum_{\theta \in \Lambda_{j}} d_{\theta} \varphi_{\theta},
$$

where $\Lambda_{j} \subset \Theta_{j}$ and $\Lambda_{j}$ will depend on $f$.

We use the notation

$$
\mathcal{X}_{\theta^{\diamond}}:=\left\{\theta \in \Theta: \theta^{\circ} \cap \theta^{\diamond} \neq \emptyset \text { and } \operatorname{level}(\theta)=\operatorname{level}\left(\theta^{\diamond}\right)\right\}
$$

for cells from the same level as $\theta^{\diamond}$ which are adjacent to it.

We start from the representation of $f_{\Gamma}$ in (3.55). We define $\tilde{\Lambda}_{0}$ as the set of all $\theta \in \Theta_{0}$ such that $\left|a_{\theta}\right|>\varepsilon^{*}\left(\left\|\varphi_{\theta}\right\|_{\infty}=1\right)$, and we set $\Lambda_{0}:=\bigcup_{\theta \in \tilde{\Lambda}_{0}} \mathcal{X}_{\theta}$. We denote

$$
\mathcal{A}_{0}:=\sum_{\theta \in \Lambda_{0}} a_{\theta} \varphi_{\theta}=: \sum_{\theta \in \Lambda_{0}} d_{\theta} \varphi_{\theta}
$$

For each $\theta^{\diamond} \in \Theta_{j}, \varphi_{\theta^{\diamond}}$ can be represented as a linear combination of $\varphi_{\theta}$ 's with $\theta \in \Theta_{j+1}$. We use this to rewrite (represent) all remaining terms $a_{\theta} \varphi_{\theta}, \theta \in \Theta_{0} \backslash \Lambda_{0}$, at the next level and add the resulting terms to the corresponding terms $a_{\theta} \varphi_{\theta}$, $\theta \in \Theta_{1}$. We denote by $d_{\theta} \varphi_{\theta}, \theta \in \Theta_{1}$, the new terms, and therefore obtain a representation of $f$ in the form

$$
f=\mathcal{A}_{0}+\sum_{\theta \in \Theta_{1}} d_{\theta} \varphi_{\theta}+\sum_{j=2}^{J} \sum_{\theta \in \Theta_{j}} a_{\theta} \varphi_{\theta} .
$$

Continuing with the next level, we define $\tilde{\Lambda}_{1}$ as the set of all $\theta \in \Theta_{1}$ such that $\left|d_{\theta}\right|>\varepsilon^{*}$, set $\Lambda_{1}:=\bigcup_{\theta \in \tilde{\Lambda}_{1}} \mathcal{X}_{\theta}$, and define $\mathcal{A}_{1}:=\sum_{\theta \in \Lambda_{1}} d_{\theta} \varphi_{\theta}$. As for the previous level, we rewrite the remaining terms $d_{\theta} \varphi_{\theta}, \theta \in \Theta_{1} \backslash \Lambda_{1}$, at the next level and 
add the resulting terms to the corresponding terms $a_{\theta} \varphi_{\theta}, \theta \in \Theta_{2}$. We obtain the following representation of $f$ :

$$
f=\mathcal{A}_{0}+\mathcal{A}_{1}+\sum_{\theta \in \Theta_{2}} d_{\theta} \varphi_{\theta}+\sum_{j=3}^{J} \sum_{\theta \in \Theta_{j}} a_{\theta} \varphi_{\theta} .
$$

We continue in this way until we reach the highest level of cells $\Theta_{J}$. At level $\Theta_{J}$, we define $\tilde{\Lambda}_{J}, \Lambda_{J}$, and $\mathcal{A}_{J}$ as above and discard all terms $d_{\theta} \varphi_{\theta}, \theta \in \Theta_{J} \backslash \Lambda_{J}$. We finally obtain our approximant $\mathcal{A}=\mathcal{A}_{\varepsilon}^{P}(f)$ in the form (3.57). We denote $\Lambda:=\Lambda_{\varepsilon}:=\bigcup_{j=0}^{J} \Lambda_{j}$ and $\tilde{\Lambda}:=\tilde{\Lambda}_{\varepsilon}:=\bigcup_{j=0}^{J} \tilde{\Lambda}_{j}$, and so $\mathcal{A}=\sum_{\theta \in \Lambda} d_{\theta} \varphi_{\theta}$.

Since we throw away only elements $d_{\theta} \varphi_{\theta}$ with $\left|d_{\theta}\right| \leq \varepsilon^{*}$ at the finest level $\Theta_{J}$, we have the estimate

$$
\left\|f_{\Gamma}-\mathcal{A}\right\|_{\infty} \leq\left\|\sum_{\theta \in \Theta_{J} \backslash \Lambda_{J}} d_{\theta} \varphi_{\theta}\right\|_{\infty} \leq \varepsilon^{*},
$$

and hence, using (3.56),

$$
\|f-\mathcal{A}\|_{\infty} \leq 2 \varepsilon^{*}=\varepsilon .
$$

This completes Step 3 and with that the description of the algorithm.

We want to point out an important distinction between the "push the error" steps in the above algorithm and the "naive" algorithm described in §3.3.1. The difference is that each time we put a significant term $d_{\theta} \varphi_{\theta}\left(\left|d_{\theta}\right|>\varepsilon^{*}\right)$ into $\mathcal{A}$ we also include the neighboring terms (i.e., from the index collection $\mathcal{X}_{\theta}$ ). This prevents our algorithm from being defeated by a situation like that described in "Trap 3" in $\S 3.3 .1$.

- Error estimation for the "push the error" algorithm. Suppose "push the error" is applied to a function $f$ with $\varepsilon>0$, and $\mathcal{A}_{\varepsilon}^{P}(f)$ is the approximant obtained: $\mathcal{A}_{\varepsilon}^{P}(f):=\sum_{\theta \in \Lambda_{\varepsilon}} d_{\theta} \varphi_{\theta}$. As in the "trim and cut" method, we use the corresponding notation

$$
n(\varepsilon):=\# \Lambda_{\varepsilon}, \mathbb{A}_{n(\varepsilon)}^{P}(f)_{\infty}:=\mathbb{A}_{n(\varepsilon)}^{P}(f, \mathcal{T})_{\infty}:=\left\|f-\mathcal{A}_{\varepsilon}^{P}(f)\right\|_{\infty},
$$

and

$$
\mathbb{A}_{n}^{P}(f)_{\infty}:=\mathbb{A}_{n}^{P}(f, \mathcal{T})_{\infty}:=\inf \left\{\mathbb{A}_{n(\varepsilon)}^{P}(f)_{\infty}: n(\varepsilon) \leq n\right\}
$$

We remark that if $f \in B_{\tau}^{\alpha}(\mathcal{T})$, then by the Embedding Theorem 2.7 it follows that $f$ is continuous. Estimates (3.59) and (3.60), established in the following theorem, imply uniform convergence of the "push the error" approximants to $f$ and provide the necessary rates of approximation by the method.

Theorem 3.11. If $f \in B_{\tau}^{\alpha}(\mathcal{T}), \alpha \geq 1, \tau:=1 / \alpha$, then for each $\varepsilon>0$,

$$
\mathbb{A}_{n(\varepsilon)}^{P}(f)_{\infty} \leq \varepsilon \quad \text { and } \quad n(\varepsilon) \leq c \varepsilon^{-\tau}\|f\|_{B_{\tau}^{\alpha}(\mathcal{T})}^{\tau},
$$

where $c=6 N_{0}^{3}$. Furthermore, we have

$$
\mathbb{A}_{n}^{P}(f)_{\infty} \leq c n^{-\alpha}\|f\|_{B_{\tau}^{\alpha}(\mathcal{T})}, \quad n=1,2, \ldots
$$

with $c=\left(6 N_{0}^{3}\right)^{\alpha}$. 
Proof. In order to prove (3.59), we first observe that the direct approximation estimate $\mathbb{A}_{n(\varepsilon)}^{P}(f)_{\infty} \leq \varepsilon$ follows from inequality (3.58) in the construction of the algorithm. Therefore it only remains to show that $\# \Lambda_{\varepsilon} \leq c \varepsilon^{-\tau}\|f\|_{B_{\tau}^{\alpha}(\mathcal{T})}^{\tau}$. Clearly,

$$
\# \Lambda_{\varepsilon} \leq\left(N_{0}+1\right)\left(\# \tilde{\Lambda}_{\varepsilon}\right),
$$

and we need only estimate the cardinality of $\tilde{\Lambda}:=\tilde{\Lambda}_{\varepsilon}$. We split $\tilde{\Lambda}$ into two disjoint sets, $\tilde{\Lambda}_{f}$ and $\tilde{\Lambda}_{r}$. We define $\tilde{\Lambda}_{f}$ as the set of all final cells in $\tilde{\Lambda}$, that is, the set of all $\theta \in \tilde{\Lambda}$ for which there is no $\theta^{\prime} \in \tilde{\Lambda}$ of a higher level sitting on $\theta$. We set $\tilde{\Lambda}_{r}:=\tilde{\Lambda} \backslash \tilde{\Lambda}_{f}$.

We shall make repeated use of the following simple lemma.

Lemma 3.12. Suppose $\mathcal{M} \subset \Theta$ satisfies the condition that cells from different levels do not have interiors that intersect. Then each $\theta \in \Theta$ may sit on at most $N_{0}+1$ cells from $\mathcal{M}$.

Proof. The simple hypothesis of the lemma just states that for a cell $\theta_{2}$ to sit on a cell $\theta_{1}$, it must be on the same level; but there can be at most $N_{0}+1$ such cells.

We first estimate the number of elements $\# \Gamma_{f}$ that arise as final cells in Step 2. For each $\theta^{\diamond} \in \Gamma_{f}$, we have, by (3.52),

$$
\varepsilon^{*}<\sum_{\theta \in \mathcal{Y}_{\theta} \diamond}\left|b_{\theta}\right| \leq\left(\sum_{\theta \in \mathcal{Y}_{\theta} \diamond}\left|b_{\theta}\right|^{\tau}\right)^{1 / \tau} \quad(\tau \leq 1) .
$$

Clearly, $\Gamma_{f}$ satisfies the hypothesis of Lemma 3.12 (see Property (i) of $\Gamma_{f}$, which is stated following (3.52) $)$, and hence each $\theta \in \Theta$ may sit on at most $N_{0}+1$ cells from $\Gamma_{f}$. Using this together with (3.62), we obtain

$$
\|f\|_{B_{\tau}^{\alpha}(\mathcal{T})}^{\tau}:=\sum_{\theta \in \Theta}\left|b_{\theta}\right|^{\tau} \geq\left(N_{0}+1\right)^{-1} \sum_{\theta^{\diamond} \in \Gamma_{f}} \sum_{\theta \in \mathcal{Y}_{\theta \diamond}}\left|b_{\theta}\right|^{\tau} \geq\left(N_{0}+1\right)^{-1}\left(\# \Gamma_{f}\right)\left(\varepsilon^{*}\right)^{\tau},
$$

which, since $\tau \leq 1$, implies

$$
\# \Gamma_{f} \leq 2\left(N_{0}+1\right) \varepsilon^{-\tau}\|f\|_{B_{\tau}^{\alpha}(\mathcal{T})}^{\tau} .
$$

We next estimate $\# \tilde{\Lambda}_{f}$, the number of final cells for the index set $\tilde{\Lambda}$ constructed in Step 3. Clearly from that construction, a cell $\theta \in \tilde{\Lambda}$ may occur only if $\theta \in \Gamma$, and hence $\tilde{\Lambda} \subset \Gamma$. On the other hand, from Step 2, for each $\theta \in \Gamma$ there exists $\theta^{\prime} \in \Gamma_{f}$ sitting on $\theta$. Therefore, for each $\theta \in \tilde{\Lambda}_{f}$ there exists $\theta^{\prime} \in \Gamma_{f}$ sitting on $\theta$. But $\tilde{\Lambda}_{f}$ satisfies the hypothesis of Lemma 3.12 (with $\mathcal{M}$ replaced by $\tilde{\Lambda}_{f}$ ), and hence a cell $\theta \in \Gamma_{f}$ may sit on at most $N_{0}+1$ cells from $\tilde{\Lambda}_{f}$. From this and (3.63), we have

$$
\# \tilde{\Lambda}_{f} \leq\left(N_{0}+1\right)\left(\# \Gamma_{f}\right) \leq 2\left(N_{0}+1\right)^{2} \varepsilon^{-\tau}\|f\|_{B_{\tau}^{\alpha}(\mathcal{T})}^{\tau} .
$$

To complete the estimate for $\# \tilde{\Lambda}$, we must estimate $\# \tilde{\Lambda}_{r}$. Suppose $\theta^{\diamond} \in \tilde{\Lambda}_{r}:=$ $\tilde{\Lambda} \backslash \tilde{\Lambda}_{f}$, and let $\theta^{\prime} \in \tilde{\Lambda}$ be a cell sitting on $\theta^{\diamond}$ with $\operatorname{level}\left(\theta^{\prime}\right)>\operatorname{level}\left(\theta^{\diamond}\right)$ and such that level $\left(\theta^{\prime}\right)$ is the minimum of the levels of all cells in $\tilde{\Lambda}$ sitting on $\theta^{\diamond}$. Such a cell exists, by the definition of $\tilde{\Lambda}_{r}$, but it is possibly not unique. We denote by $\mathcal{Z}_{\theta^{\circ}}$ the set of all $\theta \in \Gamma$ which, while "pushing the error" from $\theta^{\diamond}$ in Step 3, have contributed to the term $d_{\theta^{\prime}} \varphi_{\theta^{\prime}}$. Due to the minimality of $\theta^{\prime}$, we see that

$$
d_{\theta^{\prime}}=d_{\theta^{\prime}} \varphi_{\theta^{\prime}}\left(v_{\theta^{\prime}}\right)=\sum_{\theta \in \mathcal{Z}_{\theta^{\diamond}}} b_{\theta} \varphi_{\theta}\left(v_{\theta^{\prime}}\right)
$$


where $v_{\theta^{\prime}}$ is the "central vertex" of $\theta^{\prime}$. Since $\theta^{\prime} \in \tilde{\Lambda}$, then $\left|d_{\theta^{\prime}}\right|>\varepsilon^{*}$, and hence, using (3.65),

$$
\varepsilon^{*}<\left|d_{\theta^{\prime}}\right| \leq\left\|\sum_{\theta \in \mathcal{Z}_{\theta^{\diamond}}} b_{\theta} \varphi_{\theta}\right\|_{\infty} \leq \sum_{\theta \in \mathcal{Z}_{\theta^{\diamond}}}\left|b_{\theta}\right| \leq\left(\sum_{\theta \in \mathcal{Z}_{\theta^{\diamond}}}\left|b_{\theta}\right|^{\tau}\right)^{1 / \tau} \quad(\tau \leq 1) .
$$

It is easily seen that each $\theta \in \mathcal{Z}_{\theta}$ s satisfies the following properties:

(a) $\theta \supset \theta^{\prime}$,

(b) $\operatorname{level}\left(\theta^{\diamond}\right)<\operatorname{level}(\theta) \leq \operatorname{level}\left(\theta^{\prime}\right)$,

(c) the "central vertex" of $\theta$ lies on $\theta^{\diamond}$, and hence $\theta$ sits on $\theta^{\diamond}$.

Property (a) follows by observing that the support of an element which is rewritten at a finer level always contains the supports of the contributing finer elements. Property (b) holds, since $\mathcal{X}_{\theta^{\diamond}} \subset \Lambda$, and hence no terms $b_{\theta} \varphi_{\theta}$ with level $(\theta) \leq \operatorname{level}\left(\theta^{\diamond}\right)$ may contribute to $d_{\theta^{\prime}} \varphi_{\theta^{\prime}}$. Note that it is possible that there are $\theta$ that satisfy properties (a)-(c) above but do not belong to $\mathcal{Z}_{\theta^{\circ}}$.

Next, we show that each $\theta \in \Gamma$ may belong to at most $N_{0}+1$ sets $\mathcal{Z}_{\theta \star}$ with $\theta^{\star} \in \tilde{\Lambda}_{r}$. Indeed, let $\theta \in \Gamma$ and suppose $\theta^{\diamond} \in \tilde{\Lambda}_{r}$ is such that $\theta \in \mathcal{Z}_{\theta^{\diamond}}$. In the following, we shall use the notation from above that involves $\theta^{\diamond}$, but we will consider such $\theta^{\diamond}$ as arbitrary in $\tilde{\Lambda}$. Let $\mathcal{M}_{\theta}$ denote the set of all $\theta^{\sharp} \in \tilde{\Lambda}$ such that $\theta \in \mathcal{Z}_{\theta^{\sharp}}$. In particular, $\theta^{\diamond} \in \mathcal{M}_{\theta}$. We fix $\mathcal{M}_{\theta}$ and show that it satisfies the hypothesis of Lemma 3.12, Indeed, let $\theta_{1}, \theta_{2} \in \mathcal{M}_{\theta}$ from different levels. But this implies $\theta \in \mathcal{Z}_{\theta_{j}}(j=1,2)$, and we may as well consider $\theta_{1}=\theta^{\diamond}$ and say $\theta_{2}=\theta^{\sharp}$, where $\operatorname{level}\left(\theta^{\sharp}\right) \neq \operatorname{level}\left(\theta^{\diamond}\right)$. Evidently, level $\left(\theta^{\sharp}\right)<\operatorname{level}\left(\theta^{\prime}\right)$, from property (b) applied to $\theta^{\sharp}$ and $\theta$.

By symmetry, we may assume level $\left(\theta^{\sharp}\right)<\operatorname{level}\left(\theta^{\diamond}\right)$. If $\left(\theta^{\sharp}\right)^{\circ} \cap\left(\theta^{\diamond}\right)^{\circ} \neq \emptyset$, then $\theta^{\diamond}$ sits on $\theta^{\sharp}$ and hence, since $\operatorname{level}(\theta)>\operatorname{level}\left(\theta^{\diamond}\right), \theta$ cannot be in $\mathcal{Z}_{\theta^{\sharp}}$, which is a contradiction. Therefore, $\left(\theta^{\sharp}\right)^{\circ} \cap\left(\theta^{\circ}\right)^{\circ}=\emptyset$, which verifies the hypothesis of Lemma 3.12.

Now that Lemma 3.12 can be applied to $\mathcal{M}_{\theta}$, then $\theta$ (as any other cell from $\Theta$ ) may sit on at most $N_{0}+1$ cells $\theta^{\star} \in \mathcal{M}_{\theta}$. Therefore, $\theta$ may belong to at most $N_{0}+1$ such sets $\mathcal{Z}_{\theta^{\star}}$ with $\theta^{\star} \in \tilde{\Lambda}_{r}$. Using this and (3.66), we obtain

$$
\|f\|_{B_{\tau}^{\alpha}(\mathcal{T})}^{\tau} \geq \sum_{\theta \in \Gamma}\left|b_{\theta}\right|^{\tau} \geq\left(N_{0}+1\right)^{-1} \sum_{\theta^{\diamond} \in \tilde{\Lambda}_{r}} \sum_{\theta \in \mathcal{Z}_{\theta^{\diamond}}}\left|b_{\theta}\right|^{\tau} \geq\left(N_{0}+1\right)^{-1}\left(\# \tilde{\Lambda}_{r}\right)\left(\varepsilon^{*}\right)^{\tau} .
$$

Therefore, it follows (recall that $\tau<1$ ) that

$$
\# \tilde{\Lambda}_{r} \leq 2\left(N_{0}+1\right) \varepsilon^{-\tau}\|f\|_{B_{\tau}^{\alpha}(\mathcal{T})}^{\tau} .
$$

We combine this estimate with (3.61) and (3.64) to obtain the desired estimate of $\# \Lambda_{\varepsilon}$ in (3.59). Estimate (3.60) follows immediately from (3.59).

The following lemma will be needed in $\S 5$.

Lemma 3.13. Let $f=f^{0}+f^{1}$, where $f=\sum_{\theta \in \Theta} b_{\theta} \varphi_{\theta}, f^{j}=\sum_{\theta \in \Theta} b_{\theta}^{j} \varphi_{\theta}(j=0,1)$, and $b_{\theta}=b_{\theta}^{0}+b_{\theta}^{1}($ all $\theta \in \Theta)$, and suppose

$$
\mathcal{N}_{j}:=\left(\sum_{\theta \in \Theta}\left|b_{\theta}^{j}\right|^{\tau_{j}}\right)^{1 / \tau_{j}}<\infty \quad(j=0,1),
$$

where $\alpha_{0}, \alpha_{1} \geq 1$ and $\tau_{0}:=1 / \alpha_{0}, \tau_{1}:=1 / \alpha_{1}$. Furthermore, suppose that "push the error" is applied using the above representation of $f$, with $\varepsilon:=\varepsilon_{0}+\varepsilon_{1}$, where 
$\varepsilon_{0}, \varepsilon_{1}>0$. Then we have

$$
\begin{gathered}
\mathbb{A}_{n\left(\varepsilon_{0}+\varepsilon_{1}\right)}^{P}(f)_{\infty} \leq \varepsilon_{0}+\varepsilon_{1}, \\
n\left(\varepsilon_{0}+\varepsilon_{1}\right) \leq c \varepsilon_{0}^{-\tau_{0}} \mathcal{N}_{0}^{\tau_{0}}+c \varepsilon_{1}^{-\tau_{1}} \mathcal{N}_{1}^{\tau_{1}},
\end{gathered}
$$

where $c=6 N_{0}^{3}$. Consequently,

$$
\mathbb{A}_{n}^{P}(f)_{\infty} \leq c_{0} n^{-\alpha_{0}} \mathcal{N}_{0}+c_{1} n^{-\alpha_{1}} \mathcal{N}_{1}, \quad n=1,2, \ldots,
$$

with $c_{j}=\left(12 N_{0}^{3}\right)^{\alpha_{j}}$.

Proof. We follow in the footsteps of the proof of Theorem 3.11. We shall use the notation from there, and only indicate the differences as they arise. We denote $\varepsilon^{*}:=\varepsilon_{0}^{*}+\varepsilon_{1}^{*}$ with $\varepsilon_{j}^{*}:=\varepsilon_{j} / 2, j=0,1$. Estimate (3.67) is immediate from the description of the algorithm.

It remains to provide estimate (3.68) for the number of terms used in the approximation. As in (3.61), we have

$$
n\left(\varepsilon_{0}+\varepsilon_{1}\right):=\# \Lambda_{\varepsilon} \leq\left(N_{0}+1\right)\left(\# \tilde{\Lambda}_{\varepsilon}\right),
$$

where we denote $\tilde{\Lambda}:=\tilde{\Lambda}_{\varepsilon}$, and $\tilde{\Lambda}_{f}$ and $\tilde{\Lambda}_{r}$ have the same definitions, proceeding exactly as in the proof of Theorem 3.11. Continuing as there, we have to estimate $\# \Gamma_{f}$. For each $\theta^{\diamond} \in \Gamma_{f}$, we have, by (3.52) and the fact that $0<\tau_{j} \leq 1(j=0,1)$, that

$$
\begin{aligned}
\varepsilon_{0}^{*}+\varepsilon_{1}^{*}=\varepsilon^{*} & <\sum_{\theta \in \mathcal{Y}_{\theta^{\diamond}}}\left|b_{\theta}\right| \leq \sum_{\theta \in \mathcal{Y}_{\theta^{\diamond}}}\left|b_{\theta}^{0}\right|+\sum_{\theta \in \mathcal{Y}_{\theta^{\diamond}}}\left|b_{\theta}^{1}\right| \\
& \leq\left(\sum_{\theta \in \mathcal{Y}_{\theta^{\diamond}}}\left|b_{\theta}^{0}\right|^{\tau_{0}}\right)^{1 / \tau_{0}}+\left(\sum_{\theta \in \mathcal{Y}_{\theta^{\diamond}}}\left|b_{\theta}^{1}\right|^{\tau_{1}}\right)^{1 / \tau_{1}} .
\end{aligned}
$$

From this, it follows that, for each $\theta^{\diamond} \in \Gamma_{f}$, at least one of

$$
\varepsilon_{0}^{*}<\left(\sum_{\theta \in \mathcal{Y}_{\theta^{\diamond}}}\left|b_{\theta}^{0}\right|^{\tau_{0}}\right)^{1 / \tau_{0}} \quad \text { or } \quad \varepsilon_{1}^{*}<\left(\sum_{\theta \in \mathcal{Y}_{\theta} \diamond}\left|b_{\theta}^{1}\right|^{\tau_{1}}\right)^{1 / \tau_{1}}
$$

must hold. We denote by $\Gamma_{f}^{0}$ and $\Gamma_{f}^{1}$ the sets of all $\theta^{\diamond} \in \Gamma_{f}$ such that the respective condition from (3.71) holds for either $j=0$ or $j=1$. For $j=0,1$, we have similarly, as in the proof of Theorem 3.11 .

$$
\mathcal{N}_{j}^{\tau_{j}}:=\sum_{\theta \in \Theta}\left|b_{\theta}^{j}\right|^{\tau_{j}} \geq\left(N_{0}+1\right)^{-1} \sum_{\theta^{\diamond} \in \Gamma_{f}^{j}} \sum_{\theta \in \mathcal{Y}_{\theta^{\diamond}}}\left|b_{\theta}^{j}\right|^{\tau_{j}} \geq\left(N_{0}+1\right)^{-1}\left(\# \Gamma_{f}^{j}\right)\left(\varepsilon_{j}^{*}\right)^{\tau_{j}},
$$

and hence $\left(\tau_{j} \leq 1\right)$

$$
\# \Gamma_{f}^{j} \leq 2\left(N_{0}+1\right) \varepsilon_{j}^{-\tau_{j}} \mathcal{N}_{j}^{\tau_{j}}
$$

Therefore,

$$
\begin{aligned}
\# \tilde{\Lambda}_{f} & \leq\left(N_{0}+1\right)\left(\# \Gamma_{f}\right) \leq\left(N_{0}+1\right)\left(\# \Gamma_{f}^{0}+\# \Gamma_{f}^{1}\right) \\
& \leq 2\left(N_{0}+1\right)^{2}\left(\varepsilon_{0}^{-\tau_{0}} \mathcal{N}_{0}^{\tau_{0}}+\varepsilon_{1}^{-\tau_{1}} \mathcal{N}_{1}^{\tau_{1}}\right) .
\end{aligned}
$$

To complete the proof, we must next estimate $\# \tilde{\Lambda}_{r}$. For each $\theta^{\diamond} \in \tilde{\Lambda}_{r}$, we define $\theta^{\prime} \in \tilde{\Lambda}$ and $\mathcal{Z}_{\theta^{\circ}}$ exactly as in the proof of Theorem 3.11. Similarly as in (3.66), we 
have

$$
\begin{aligned}
\varepsilon_{0}^{*}+\varepsilon_{1}^{*}=\varepsilon^{*} & <\sum_{\theta \in \mathcal{Z}_{\theta^{\diamond}}}\left|b_{\theta}\right| \leq \sum_{\theta \in \mathcal{Z}_{\theta^{\diamond}}}\left|b_{\theta}^{0}\right|+\sum_{\theta \in \mathcal{Z}_{\theta}}\left|b_{\theta}^{1}\right| \\
& \leq\left(\sum_{\theta \in \mathcal{Z}_{\theta^{\diamond}}}\left|b_{\theta}^{0}\right|^{\tau_{0}}\right)^{1 / \tau_{0}}+\left(\sum_{\theta \in \mathcal{Z}_{\theta}}\left|b_{\theta}^{1}\right|^{\tau_{1}}\right)^{1 / \tau_{1}} .
\end{aligned}
$$

From this, it follows that, for each $\theta^{\diamond} \in \tilde{\Lambda}_{r}$, at least one of

$$
\varepsilon_{0}^{*}<\left(\sum_{\theta \in \mathcal{Z}_{\theta} \diamond}\left|b_{\theta}^{0}\right|^{\tau_{0}}\right)^{1 / \tau_{0}}
$$

or

$$
\varepsilon_{1}^{*}<\left(\sum_{\theta \in \mathcal{Z}_{\theta} \diamond}\left|b_{\theta}^{1}\right|^{\tau_{1}}\right)^{1 / \tau_{1}}
$$

must hold. We denote by $\tilde{\Lambda}_{r}^{0}$ and $\tilde{\Lambda}_{r}^{1}$ the sets of all $\theta^{\diamond} \in \tilde{\Lambda}_{r}$ for which (3.73) and (3.74) hold, respectively. As in the proof of Theorem 3.11, each $\theta \in \Theta$ may belong to at most $N_{0}+1$ sets $\mathcal{Z}_{\theta^{\diamond}}, \theta^{\diamond} \in \tilde{\Lambda}_{r}$. Therefore, for $j=0,1$,

$$
\mathcal{N}_{j}^{\tau_{j}} \geq \sum_{\theta \in \Gamma}\left|b_{\theta}^{j}\right|^{\tau_{j}} \geq\left(N_{0}+1\right)^{-1} \sum_{\theta^{\diamond} \in \tilde{\Lambda}_{r}^{j}} \sum_{\theta \in \mathcal{Z}_{\theta^{\diamond}}}\left|b_{\theta}^{j}\right|^{\tau_{j}} \geq\left(N_{0}+1\right)^{-1}\left(\# \tilde{\Lambda}_{r}^{j}\right)\left(\varepsilon_{j}^{*}\right)^{\tau_{j}},
$$

and hence

$$
\# \tilde{\Lambda}_{r}^{j} \leq 2\left(N_{0}+1\right) \varepsilon_{j}^{-\tau_{j}} \mathcal{N}_{j}^{\tau_{j}}, \quad j=0,1
$$

Therefore,

$$
\# \tilde{\Lambda}_{r} \leq \# \tilde{\Lambda}_{r}^{0}+\# \tilde{\Lambda}_{r}^{1} \leq 2\left(N_{0}+1\right)\left(\varepsilon_{0}^{-\tau_{0}} \mathcal{N}_{0}^{\tau_{0}}+\varepsilon_{1}^{-\tau_{1}} \mathcal{N}_{1}^{\tau_{1}}\right) .
$$

This estimate, together with (3.70) and (3.72), implies (3.68) (since $N_{0} \geq 3$ ). Estimate (3.69) follows by using $\varepsilon_{j}:=(2 c)^{\alpha_{j}} n^{-\alpha_{j}} \mathcal{N}_{j}(j=0,1)$ in (3.67) and (3.68) to obtain $n\left(\varepsilon_{0}+\varepsilon_{1}\right) \leq n$, and so $\mathbb{A}_{n}^{P}(f)_{\infty} \leq \mathbb{A}_{n\left(\varepsilon_{0}+\varepsilon_{1}\right)}^{P}(f)_{\infty} \leq \varepsilon_{0}+\varepsilon_{1}$.

\section{Best $n$-TERM Courant ElEMEnt approximation}

In this section, we assume that $\mathcal{T}$ is a locally regular triangulation of a bounded polygonal domain $E$ with parameters $N_{0}, M_{0}, r, \rho, \delta$, and $\# \mathcal{T}_{0}$ (see \$2.1). We denote by $\Phi_{\mathcal{T}}$ the collection of all Courant elements $\varphi_{\theta}$ generated by $\mathcal{T}$. Notice that $\Phi_{\mathcal{T}}$ is not a basis; $\Phi_{\mathcal{T}}$ is redundant. We consider nonlinear $n$-term approximation in $L_{p}(E)(0<p \leq \infty)$ from $\Phi_{\mathcal{T}}$, where we identify $L_{\infty}(E)$ as $C(E)$. Our main goal is to characterize the approximation spaces generated by this approximation, with emphasis on the case $p=\infty$. We let $\Sigma_{n}(\mathcal{T})$ denote the nonlinear set consisting of all continuous piecewise linear functions $S$ of the form

$$
S=\sum_{\theta \in \mathcal{M}} a_{\theta} \varphi_{\theta},
$$

where $\mathcal{M} \subset \Theta(\mathcal{T}), \# \mathcal{M} \leq n$, and $\mathcal{M}$ may vary with $S$. We denote by $\sigma_{n}(f, \mathcal{T})_{p}$ the best $L_{p}$-approximation of $f \in L_{p}(E)$ from $\Sigma_{n}(\mathcal{T})$ :

$$
\sigma_{n}(f, \mathcal{T})_{p}:=\inf _{S \in \Sigma_{n}(\mathcal{T})}\|f-S\|_{p}
$$

In order to characterize the approximation spaces generated by $\left(\sigma_{n}(f, \mathcal{T})_{p}\right)$, we begin in this section by first proving a companion pair of Jackson and Bernstein 
inequalities, and then follow with the usual techniques of interpolation of operators (see for example [6], 15], [13]).

In the following, we assume in general that $0<p \leq \infty$, and that $\alpha \geq 1$ for $p=\infty$ and $\alpha>0$ if $p<\infty$; in either case we set $1 / \tau:=\alpha+1 / p$.

Theorem 4.1 (Jackson estimate). If $f \in B_{\tau}^{\alpha}(\mathcal{T})$, then

$$
\sigma_{n}(f, \mathcal{T})_{p} \leq c n^{-\alpha}\|f\|_{B_{\tau}^{\alpha}(\mathcal{T})},
$$

where $c$ depends only on $\alpha, p$ and the parameters of $\mathcal{T}$.

Proof. Estimate (4.1) follows from any of our constructive algorithms as formulated in the corresponding Theorems 3.1, 3.7, 3.9, or 3.11.

Theorem 4.2 (Bernstein estimate). If $S \in \Sigma_{n}(\mathcal{T})$, then

$$
\|S\|_{B_{\tau}^{\alpha}(\mathcal{T})} \leq c n^{\alpha}\|S\|_{p}
$$

where $c$ depends only on $\alpha, p$, and the parameters of $\mathcal{T}$.

Proof. We shall prove estimate (4.2) only in the case $p=\infty$. For the proof when $p<\infty$, see [11]. Suppose $S \in \Sigma_{n}^{k}(\mathcal{T})$ and $S=: \sum_{\theta \in \mathcal{M}} c_{\theta} \varphi_{\theta}$, where $\mathcal{M} \subset \Theta(\mathcal{T})$ and $\# \mathcal{M} \leq n$. Let $\Lambda$ be the set of all triangles $\Delta \in \mathcal{T}$ that are involved in all cells $\theta \in \mathcal{M}$. Then $S=\sum_{\Delta \in \Lambda} S_{\Delta}$, where $S_{\Delta}=: \mathbb{1}_{\Delta} \cdot P_{\Delta}, P_{\Delta}$ a linear polynomial. Evidently, $\# \Lambda \leq N_{0} \# \mathcal{M} \leq c n$.

We shall utilize the natural tree structure in $\mathcal{T}$ induced by the inclusion relation: Each triangle $\Delta \in \mathcal{T}_{m}$ has (contains) $\leq M_{0}$ children in $\mathcal{T}_{m+1}$ and one parent in $\mathcal{T}_{m-1}$, if $m \geq 1$. Let $\Gamma_{0}$ be the set of all $\Delta \in \mathcal{T}$ such that $\Delta \supset \Delta^{\prime}$ for some $\Delta^{\prime} \in \Lambda$. We denote by $\Gamma_{b}$ the set of all branching triangles in $\Gamma_{0}$ (triangles with more than one child in $\Gamma_{0}$ ) and by $\Gamma_{b}^{\prime}$ the set of all children of branching triangles in $\mathcal{T}$ (which may or may not belong to $\Gamma_{0}$ ). Now, we extend $\Gamma_{0}$ to $\Gamma:=\Gamma_{0} \cup \Gamma_{b}^{\prime}$. We also extend $\Lambda$ to $\tilde{\Lambda}:=\Lambda \cup \Gamma_{b} \cup \Gamma_{b}^{\prime}$. In addition, we introduce the following subsets of $\Gamma$ : the set $\Gamma_{f}$ of all final triangles in $\Gamma$ (triangles in $\Gamma$ containing no other triangles in $\Gamma)$, the set $\left(\Gamma_{0}\right)_{f}$ of the final triangles in $\Gamma_{0}$, and the set $\Gamma_{c h}:=\Gamma \backslash \tilde{\Lambda}$ of all chain triangles. Note that each triangle $\Delta \in \Gamma_{c h}$ has exactly one child in $\Gamma$. We may argue as we did for trees of cells in (3.9) that the number of branching triangles does not exceed the number of final triangles, $\# \Gamma_{b} \leq \#\left(\Gamma_{0}\right)_{f}$, and since $\left(\Gamma_{0}\right)_{f} \subset \Lambda$, then $\# \Gamma_{b} \leq c n$. Using this, we have $\# \Gamma_{b}^{\prime} \leq M_{0} \# \Gamma_{b} \leq c n, \# \Gamma_{f} \leq \# \Lambda+\# \Gamma_{b}^{\prime} \leq c n$, and $\# \tilde{\Lambda} \leq \# \Lambda+\# \Gamma_{b}+\# \Gamma_{b}^{\prime} \leq c n$. Keep in mind, however, that $\# \Gamma_{c h}$ can be much larger than $n$.

We next estimate $|S|_{B_{\tau}^{\alpha}(\mathcal{T})}^{\tau}:=\sum_{\Delta \in \mathcal{T}}|\Delta|^{-1} S S_{\Delta}(S)_{\tau}^{\tau}$, where $\tau:=1 / \alpha$ (see (2.5)

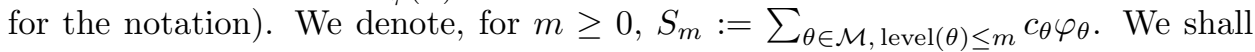
use that, for $\Delta \in \mathcal{T}_{m}$,

$$
S S_{\Delta}(S)_{\tau}=S S_{\Delta}\left(S-S_{m}\right)_{\tau} \leq\left\|S-S_{m}\right\|_{L_{\tau}\left(\Omega_{\Delta}\right)}
$$

and, also, $S S_{\Delta}(S)_{\tau} \leq\|S\|_{L_{\tau}\left(\Omega_{\Delta}\right)}$. Recall that $\Omega_{\Delta}$ is the union of the collection of all triangles from the same level as $\Delta$ and which share a vertex. We denote

$$
\mathcal{H}_{m}:=\left\{\Delta \in \mathcal{T}_{m}: \Delta \subset \Omega_{\Delta^{\prime}} \text { for some } \Delta^{\prime} \in \tilde{\Lambda} \cap \mathcal{T}_{m}\right\} \quad \text { and } \quad \mathcal{H}:=\bigcup_{m \geq 0} \mathcal{H}_{m} .
$$

Evidently, $\# \mathcal{H}_{m} \leq 3 N_{0} \# \tilde{\Lambda} \leq c n$ (the valence of each vertex is $\leq N_{0}$ ). We consider two possibilities for each $\Delta \in \mathcal{T}$ : (a) $\Delta \in \mathcal{H}$, or (b) $\Delta \in \mathcal{T} \backslash \mathcal{H}$ : 
(a) If $\Delta \in \mathcal{H}_{m}$, then $\Omega_{\Delta} \supset \Delta^{\prime}$ for some $\Delta^{\prime} \in \tilde{\Lambda} \cap \mathcal{T}_{m}$. Using (2.3), we obtain

$$
|\Delta|^{-1} S S_{\Delta}(S)_{\tau}^{\tau} \leq|\Delta|^{-1}\|S\|_{L_{\tau}\left(\Omega_{\Delta}\right)}^{\tau} \leq|\Delta|^{-1}\left|\Omega_{\Delta}\right|\|S\|_{\infty}^{\tau} \leq c\|S\|_{\infty}^{\tau} .
$$

Therefore, by summing over all $m \geq 0$, we obtain in this case

$$
\begin{aligned}
\sum_{\Delta \in \mathcal{H}}|\Delta|^{-1} S S_{\Delta}(S)_{\tau}^{\tau} & =\sum_{m \geq 0} \sum_{\Delta \in \mathcal{H}_{m}}|\Delta|^{-1} S S_{\Delta}(S)_{\tau}^{\tau} \\
& \leq c\|S\|_{\infty}^{\tau} \sum_{m \geq 0} \# \mathcal{H}_{m} \\
& =c\|S\|_{\infty}^{\tau} \# \mathcal{H} \leq c n\|S\|_{\infty}^{\tau} .
\end{aligned}
$$

(b) Let $\Delta \in \mathcal{T}_{m} \backslash \mathcal{H}_{m}$. Then $\Omega_{\Delta}=: \bigcup_{j=1}^{n_{\Delta}} \Delta_{j}$ for some $\Delta_{j} \in\left(\Gamma_{c h} \cap \mathcal{T}_{m}\right) \cup\left(\mathcal{T}_{m} \backslash \Gamma\right)$, $j=1, \ldots, n_{\Delta}$, with $n_{\Delta} \leq 3 N_{0}$. We have, using (4.3),

$$
S S_{\Delta}(S)_{\tau}^{\tau}=S S_{\Delta}\left(S-S_{m}\right)_{\tau}^{\tau} \leq \sum_{j=1}^{n_{\Delta}}\left\|S-S_{m}\right\|_{L_{\tau}\left(\Delta_{j}\right)}^{\tau} .
$$

Note that if $\Delta_{j} \in \mathcal{T}_{m} \backslash \Gamma$, then $\left.S\right|_{\Delta_{j}}=\left.S_{m}\right|_{\Delta_{j}}$ and hence $\left\|S-S_{m}\right\|_{L_{\tau}\left(\Delta_{j}\right)}=0$. Suppose $\Delta_{j} \in \Gamma_{c h} \cap \mathcal{T}_{m}$. For each $\Delta \in \Gamma_{c h}$, we shall denote by $\tilde{\Delta}(\tilde{\Delta} \neq \Delta)$ the unique largest triangle of $\tilde{\Lambda}$ contained in $\Delta$. Clearly, we have $\left.S\right|_{\Delta_{j} \backslash \tilde{\Delta}_{j}}=\left.S_{m}\right|_{\Delta_{j} \backslash \tilde{\Delta}_{j}}=$ $\mathbb{1}_{\Delta_{j} \backslash \tilde{\Delta}_{j}} \cdot P_{\Delta_{j}}$ and $\left.S_{m}\right|_{\Delta_{j}}=\mathbb{1}_{\Delta_{j}} \cdot P_{\Delta_{j}}$, where $P_{\Delta_{j}}$ is a linear polynomial. Therefore,

$$
\begin{aligned}
\left\|S-S_{m}\right\|_{L_{\tau}\left(\Delta_{j}\right)}^{\tau} & =\left\|S-S_{m}\right\|_{L_{\tau}\left(\tilde{\Delta}_{j}\right)}^{\tau} \\
& \leq c\left|\tilde{\Delta}_{j}\right|\left(\|S\|_{\infty}^{\tau}+\left\|P_{\Delta_{j}}\right\|_{L_{\infty}\left(\tilde{\Delta}_{j}\right)}^{\tau}\right) \leq c\left|\tilde{\Delta}_{j}\right|\|S\|_{\infty}^{\tau},
\end{aligned}
$$

where we used that $\left\|P_{\Delta_{j}}\right\|_{L_{\infty}\left(\tilde{\Delta}_{j}\right)} \leq\left\|P_{\Delta_{j}}\right\|_{L_{\infty}\left(\Delta_{j}\right)} \leq c\left\|P_{\Delta_{j}}\right\|_{L_{\infty}\left(\Delta_{j} \backslash \tilde{\Delta}_{j}\right)} \leq c\|S\|_{\infty}$, applying Lemma 2.1 From the above, it follows that

$$
|\Delta|^{-1} S S_{\Delta}(S)_{\tau}^{\tau} \leq c\|S\|_{\infty}^{\tau} \sum_{1 \leq j \leq n_{\Delta}, \Delta_{j} \in \Gamma_{c h} \cap \mathcal{T}_{m}}\left|\tilde{\Delta}_{j}\right| /\left|\Delta_{j}\right|
$$

and hence

$$
\sum_{\Delta \in \mathcal{T}_{m} \backslash \mathcal{H}_{m}}|\Delta|^{-1} S S_{\Delta}(S)_{\tau}^{\tau} \leq c\|S\|_{\infty}^{\tau} \sum_{\Delta \in \Gamma_{c h} \cap \mathcal{T}_{m}}|\tilde{\Delta}| /|\Delta| .
$$

Summing over $m \geq 0$ in this case as well, we find that

$$
\begin{aligned}
\sum_{\Delta \in \mathcal{T} \backslash \mathcal{H}}|\Delta|^{-1} S S_{\Delta}(S)_{\tau}^{\tau} & \leq c\|S\|_{\infty}^{\tau} \sum_{\Delta \in \Gamma_{c h}}|\tilde{\Delta}| /|\Delta| \\
& \leq c\|S\|_{\infty}^{\tau} \sum_{\Delta^{\prime} \in \tilde{\Lambda}} \sum_{\Delta \in \mathcal{T}, \Delta \supset \Delta^{\prime}}\left|\Delta^{\prime}\right| /|\Delta| \\
& \leq c\|S\|_{\infty}^{\tau} \sum_{\Delta^{\prime} \in \tilde{\Lambda}} \sum_{j=0}^{\infty} \rho^{j} \leq c\|S\|_{\infty}^{\tau} \# \tilde{\Lambda} \leq c n\|S\|_{\infty}^{\tau},
\end{aligned}
$$

where we have once switched the order of summation and used that $\left|\Delta^{\prime}\right| \leq \rho|\Delta|$ if $\Delta^{\prime}$ is a child of $\Delta$ (see (2.2)).

Combining inequalities (4.4) and (4.5), we obtain $|S|_{B_{\tau}^{\alpha}(\mathcal{T})}^{\tau} \leq c n\|S\|_{\infty}^{\tau}$, which is equivalent to (4.2). 
We define the approximation space $A_{q}^{\gamma}\left(L_{p}\right):=A_{q}^{\gamma}\left(L_{p}, \mathcal{T}\right)$ generated by the $n$ term Courant element approximation to be the set of all functions $f \in L_{p}(E)$ such that

$$
\|f\|_{A_{q}^{\gamma}\left(L_{p}\right)}:=\|f\|_{p}+\left(\sum_{n=1}^{\infty}\left(n^{\gamma} \sigma_{n}(f, \mathcal{T})_{p}\right)^{q} \frac{1}{n}\right)^{1 / q}<\infty
$$

with the usual modification when $q=\infty$.

For a fixed LR-triangulation $\mathcal{T}$, we denote by $K(f, t):=K\left(f, t ; L_{p}, B_{\tau}^{\alpha}(\mathcal{T})\right)$ the $K$-functional as defined in (2.30). The Jackson and Bernstein estimates from Theorem 4.1 and Theorem 4.2 yield (see, e.g., Theorem 3.16 of [15] and its proof) the following direct and inverse estimates:

$$
\sigma_{n}(f, \mathcal{T})_{p} \leq c K\left(f, n^{-\alpha}\right)
$$

and

$$
K\left(f, n^{-\alpha}\right) \leq c n^{-\alpha}\left(\|f\|_{p}+\left(\sum_{k=1}^{n} \frac{1}{k}\left(k^{\alpha} \sigma_{k}(f, \mathcal{T})_{p}\right)^{p^{*}}\right)^{1 / p^{*}}\right), \quad p^{*}:=\min \{p, 1\},
$$

where $c$ depends only on $\alpha, p$, and the parameters of $\mathcal{T}$.

The following characterization of the approximation spaces $A_{q}^{\gamma}\left(L_{p}, \mathcal{T}\right)$ is immediate from the inequalities (4.7) and (4.8), using the observation (2.31):

Theorem 4.3. If $0<\gamma<\alpha$ and $0<q \leq \infty$, then

$$
A_{q}^{\gamma}\left(L_{p}, \mathcal{T}\right)=\left(L_{p}, B_{\tau}^{\alpha}(\mathcal{T})\right)_{\frac{\gamma}{\alpha}, q}
$$

with equivalent norms.

The next result establishes an important (continuous) embedding, which will be needed in $\S 5$ in order to identify the approximation spaces (the ones determined by the algorithms, as well as best $n$-term Courant element approximation) as $B$-spaces.

Theorem 4.4. Suppose our standing assumptions hold, i.e., $\alpha>1$ if $p=\infty$ and $\alpha>0$ if $p<\infty$. If we let $1 / \tau:=\alpha+1 / p$, then $A_{\tau}^{\alpha}\left(L_{p}, \mathcal{T}\right) \subset B_{\tau}^{\alpha}(\mathcal{T})$ and

$$
\|f\|_{B_{\tau}^{\alpha}(\mathcal{T})} \leq c\|f\|_{A_{\tau}^{\alpha}\left(L_{p}, \mathcal{T}\right)}
$$

where $c$ depends only on $\alpha, p$, and the parameters of $\mathcal{T}$.

Proof. We shall prove (4.9) only in the case $p=\infty$, proceeding similarly as in [7]. For a proof in the case $0<p<\infty$, see [3]. Suppose $f \in A_{\tau}^{\alpha}\left(L_{\infty}, \mathcal{T}\right)$, and let $S_{m} \in \Sigma_{m}(\mathcal{T})$ be such that

$$
\left\|f-S_{m}\right\|_{\infty} \leq 2 \sigma_{m}(f, \mathcal{T})_{\infty}
$$

Since $\sigma_{m}(f, \mathcal{T})_{\infty} \rightarrow 0$, we have $f=S_{1}+\sum_{\nu=1}^{\infty}\left(S_{2^{\nu}}-S_{2^{\nu-1}}\right)$ with the series converging uniformly, and hence $(\tau<1)$

$$
\|f\|_{B_{\tau}^{\alpha}(\mathcal{T})}^{\tau} \leq\left\|S_{1}\right\|_{B_{\tau}^{\alpha}(\mathcal{T})}^{\tau}+\sum_{\nu=1}^{\infty}\left\|S_{2^{\nu}}-S_{2^{\nu-1}}\right\|_{B_{\tau}^{\alpha}(\mathcal{T})}^{\tau} .
$$

We apply the Bernstein estimate from Theorem 4.2 to $S_{2^{\nu}}-S_{2^{\nu-1}} \in \Sigma_{2^{\nu+1}}(\mathcal{T})$ to obtain

$$
\left\|S_{2^{\nu}}-S_{2^{\nu-1}}\right\|_{B_{\tau}^{\alpha}(\mathcal{T})} \leq c 2^{\nu \alpha}\left\|S_{2^{\nu}}-S_{2^{\nu-1}}\right\|_{\infty} \leq c 2^{\nu \alpha}\left(\sigma_{2^{\nu}}(f, \mathcal{T})_{\infty}+\sigma_{2^{\nu-1}}(f, \mathcal{T})_{\infty}\right)
$$


and similarly

$$
\left\|S_{1}\right\|_{B_{\tau}^{\alpha}(\mathcal{T})} \leq c\left(\|f\|_{\infty}+\sigma_{1}(f, \mathcal{T})_{\infty}\right) .
$$

Substituting the above in (4.11), we find that

$$
\|f\|_{B_{\tau}^{\alpha}(\mathcal{T})}^{\tau} \leq c\|f\|_{\infty}^{\tau}+c \sum_{\nu=1}^{\infty}\left(2^{\nu \alpha} \sigma_{2^{\nu}}(f, \mathcal{T})_{\infty}\right)^{\tau} \leq c\|f\|_{A_{\tau}^{\alpha}\left(L_{\infty}, \mathcal{T}\right)}^{\tau} .
$$

\section{Approximation SPaCES fOR ALgOrithms}

Our goal in this section is to show that the algorithms that we developed and explored in $\S 3$ achieve (in a certain sense) the rate of convergence of the best $n$ term Courant element approximation. We shall utilize the characterization of the approximation spaces

$$
A_{q}^{\gamma}\left(L_{p}, \mathcal{T} ; \sigma\right):=A_{q}^{\gamma}\left(L_{p}, \mathcal{T}\right)
$$

from the previous section (see Theorems 4.3 and 4.4). We shall denote by $A_{q}^{\gamma}\left(L_{p}, \mathcal{T} ; \mathbb{A}^{T}\right), A_{q}^{\gamma}\left(L_{p}, \mathcal{T} ; \mathbb{A}^{T C}\right)$, and $A_{q}^{\gamma}\left(L_{p}, \mathcal{T} ; \mathbb{A}^{P}\right)$ the approximation spaces generated by the "threshold", "trim and cut", and "push the error" algorithms, respectively. Namely, $f \in A_{q}^{\gamma}\left(L_{p}, \mathcal{T} ; \mathbb{A}\right)$, where $\mathbb{A}$ is $\mathbb{A}^{T}, \mathbb{A}^{T C}$ or $\mathbb{A}^{P}$, if $f \in L_{p}(E)$ and

$$
\|f\|_{A_{q}^{\gamma}\left(L_{p}, \mathcal{T} ; \mathbb{A}\right)}:=\|f\|_{p}+\left(\sum_{n=1}^{\infty}\left(n^{\gamma} \mathbb{A}_{n}(f, \mathcal{T})_{p}\right)^{q} \frac{1}{n}\right)^{1 / q}<\infty,
$$

with the usual modification when $q=\infty$ (it is not quite a norm).

Theorem 5.1. Let $\mathcal{T}$ be an LR-triangulation of a bounded polygonal domain $E \subset$ $\mathbb{R}^{2}$.

(a) If $p=\infty, \alpha>1$, and $\tau:=1 / \alpha$, then

$$
A_{\tau}^{\alpha}\left(L_{\infty}, \mathcal{T} ; \mathbb{A}^{P}\right)=A_{\tau}^{\alpha}\left(L_{\infty}, \mathcal{T} ; \mathbb{A}^{T C}\right)=A_{\tau}^{\alpha}\left(L_{\infty}, \mathcal{T} ; \sigma\right)=B_{\tau}^{\alpha}(\mathcal{T})
$$

with equivalent "norms".

(b) If $0<p<\infty, \alpha>0$, and $\tau:=(\alpha+1 / p)^{-1}$, then

$$
A_{\tau}^{\alpha}\left(L_{p}, \mathcal{T} ; \mathbb{A}^{T C}\right)=A_{\tau}^{\alpha}\left(L_{p}, \mathcal{T} ; \mathbb{A}^{T}\right)=A_{\tau}^{\alpha}\left(L_{p}, \mathcal{T} ; \sigma\right)=B_{\tau}^{\alpha}(\mathcal{T})
$$

with equivalent "norms", where "trim and cut" is applied with parameter $\tau \leq \varrho<p$.

Proof. (a) Let $p=\infty$. We let $\mathbb{A}_{n}(f)_{\infty}$ denote $\mathbb{A}_{n}^{P}(f)_{\infty}$ or $\mathbb{A}_{n}^{T C}(f)_{\infty}$, and $A_{\tau}^{\alpha}\left(L_{\infty} ; \mathbb{A}\right)$ denote the approximation space generated by the corresponding algorithm. Suppose $\|f\|_{A_{\tau}^{\alpha}\left(L_{\infty} ; \mathbb{A}\right)}<\infty$. Evidently, $\sigma_{n}(f)_{\infty} \leq \mathbb{A}_{n}(f)_{\infty}$, and hence, using Theorem 4.4.

$$
\|f\|_{B_{\tau}^{\alpha}} \leq c\|f\|_{A_{\tau}^{\alpha}\left(L_{\infty} ; \sigma\right)} \leq c\|f\|_{A_{\tau}^{\alpha}\left(L_{\infty} ; \mathbb{A}\right)} .
$$

It remains to show that if $\|f\|_{B_{\tau}^{\alpha}}<\infty$, then

$$
\|f\|_{A_{\tau}^{\alpha}\left(L_{\infty} ; \mathbb{A}\right)} \leq c\|f\|_{B_{\tau}^{\alpha}} .
$$

For the proof of this estimate, we shall employ Lemmas 3.8 and 3.13 . Since they are identical, it does not matter if we prove (5.3) for "push the error" or for "trim and cut".

Suppose $f=\sum_{\theta \in \Theta} b_{\theta} \varphi_{\theta}$ is the representation of $f$ that is used while "push the error" or "trim and cut" is applied. We have

$$
\|f\|_{B_{\tau}^{\alpha}}:=\left(\sum_{\theta \in \Theta}\left|b_{\theta}\right|^{\tau}\right)^{1 / \tau}, \quad \tau:=1 / \alpha, \alpha>1 .
$$


Next, we use a well-known interpolation technique. We choose $\alpha_{0}, \alpha_{1}, \tau_{0}$, and $\tau_{1}$ as follows: $1=\alpha_{1}<\alpha<\alpha_{0}$ and $\tau_{0}:=1 / \alpha_{0}, \tau_{1}:=1 / \alpha_{1}$. Hence $0<\tau_{0}<\tau<\tau_{1}=1$. Now let $\left(\left|b_{\theta_{j}}\right|\right)_{j=1}^{\infty}$ be the decreasing rearrangement of the sequence $\left(\left|b_{\theta}\right|\right)_{\theta \in \Theta}$, i.e., indexed so that

$$
\left|b_{\theta_{1}}\right| \geq\left|b_{\theta_{2}}\right| \geq \cdots
$$

We fix $\nu \geq 0$, and denote $f^{0}:=\sum_{j=1}^{2^{\nu}} b_{\theta_{\nu}} \varphi_{\theta_{\nu}}$ and $f^{1}:=\sum_{j=2^{\nu}+1}^{\infty} b_{\theta_{\nu}} \varphi_{\theta_{\nu}}$. In going further, we apply Lemma 3.8 or Lemma 3.13 to $f=f^{0}+f^{1}$, from above, to obtain

$$
\mathbb{A}_{2^{\nu}}(f)_{\infty} \leq c 2^{-\nu \alpha_{0}}\left(\sum_{j=1}^{2^{\nu}}\left|b_{\theta_{j}}\right|^{\tau_{0}}\right)^{1 / \tau_{0}}+c 2^{-\nu} \sum_{j=2^{\nu}+1}^{\infty}\left|b_{\theta_{j}}\right| .
$$

Using property (5.4) and the facts that $\tau=1 / \alpha, 1<\alpha<\alpha_{0}$, and $\tau_{0}=1 / \alpha_{0}$, we infer

$$
\begin{aligned}
\sum_{\nu=0}^{\infty}\left(2^{\nu \alpha} \mathbb{A}_{2^{\nu}}(f)_{\infty}\right)^{\tau} \leq & c \sum_{\nu=0}^{\infty}\left[2^{-\nu\left(\alpha_{0}-\alpha\right) \tau_{0}} \sum_{k=0}^{\nu} 2^{k}\left|b_{\theta_{2^{k}}}\right|^{\tau_{0}}\right]^{\tau / \tau_{0}} \\
& +c \sum_{\nu=0}^{\infty}\left[2^{\nu(\alpha-1)} \sum_{k=\nu}^{\infty} 2^{k}\left|b_{\theta_{2^{k}}}\right|\right]^{\tau} \\
\leq & \left.c \sum_{k=0}^{\infty} 2^{k}\left|b_{\theta_{2^{k}}} \tau^{\tau} \leq c \sum_{\theta \in \Theta}\right| b_{\theta}\right|^{\tau}=c\|f\|_{B_{\tau}^{\alpha}}^{\tau},
\end{aligned}
$$

where we used the well-known Hardy inequalities, namely, we applied the inequality from Lemma 3.10 in [15] to estimate the first sum and Lemma 3.4 from [6] to the second term.

(b) For $0<p<\infty$, the proof of (5.2) is similar to the proof of (5.1). The only difference is that the appropriate roles of Lemmas 3.8 or 3.13 are now played by Lemmas 3.2 or [3.10. We omit the details.

\section{Concluding Remarks}

Our primary goal in the present article is to quantify the nonlinear $n$-term approximation from Courant elements and use it to develop algorithms capable of achieving the rate of the best approximation. This is closely related to the fundamental question in nonlinear approximation of how to measure the smoothness of the functions. As we show in this article, for $n$-term Courant element approximation when the triangulation $\mathcal{T}$ is fixed, it is natural to measure the smoothness via the scale of the $B$-spaces $B_{\tau}^{\alpha}(\mathcal{T})$. The use of these spaces allows one to characterize the approximation spaces for any rate of convergence $\alpha>0$. It also enables us to develop algorithms which attain the rate of the best approximation.

It is natural to add another degree of nonlinearity to the approximation by allowing the triangulation $\mathcal{T}$ to vary. Thus a function $f$ should be considered smooth of order $\alpha>0$ if $\inf _{\mathcal{T}}\|f\|_{B_{\tau}^{\alpha}(\mathcal{T})}<\infty$, where the infimum is taken over all LR-triangulations $\mathcal{T}$ (with fixed parameters). Therefore the rate of $n$-term Courant element approximation to $f$ is roughly $O\left(n^{-\alpha}\right)$. Summarizing, our approximation scheme proceeds as follows: (i) for a given function $f$, find a triangulation $\mathcal{T}_{f}$ and 
a $B$-space $B_{\tau}^{\alpha}\left(\mathcal{T}_{f}\right)$ in which $f$ exhibits the most smoothness, (ii) find an optimal representation of $f$ in terms of Courant elements from $\Phi_{\mathcal{T}}$, and (iii) run an algorithm that achieves the rate of the best $n$-term Courant element approximation. The first step in this scheme is the most complicated one. We do not have an efficient solution for this as yet. In the simpler case of nonlinear approximation by piecewise polynomials over dyadic partitions, this problem, however, has a complete and efficient solution 14. As we show, once the triangulation $\mathcal{T}$ is determined, the remaining two steps are now well understood and have efficient solutions in both theoretical and practical senses.

The three algorithms that we develop and explore in this article provide solutions of the problem under appropriate conditions. A common feature of these algorithms is the first step, a nontrivial decomposition from the redundant collection of all Courant elements from $\Phi_{\mathcal{T}}$. After this initial step, however, they take three different routes. The "threshold" algorithm is completely unstructured but easy to implement. The drawback of this procedure is that it is not valid in the case of the uniform norm, and as a consequence it does not perform well in $L_{p}$ for $p$ large. The "trim and cut" algorithm is valid for $L_{p}, 0<p \leq \infty$, but it is overstructured and as a result the performance suffers. The "push the error" algorithm appears to be the preferred approximation method.

The algorithms that we develop in this article are not restricted to $n$-term Courant element approximation. They can be applied immediately to the approximation from (discontinuous) piecewise approximation over multilevel triangulations (for the precise setting, see [11]). In this case the role of the $B$-spaces $B_{\tau}^{\alpha}(\mathcal{T})$ should be played by the skinny $B$-spaces $\mathcal{B}_{\tau}^{\alpha}(\mathcal{T})$, introduced in (2.37). The results are similar, but simplify considerably. We omit the details.

Furthermore, these algorithms can easily be adapted to nonlinear $n$-term approximation by smooth piecewise polynomial basis functions such as those considered in [3] and, in particular, by box splines. The main difference would be that one should use the corresponding $B$-spaces, developed in [3], but proceeding in a similar manner to this paper.

It is natural to use (wavelet or prewavelet) bases in nonlinear approximation, and specifically for approximation in $L_{p}(1<p<\infty)$. We are not aware of compactly supported wavelets (prewavelets) generated by Courant elements or smoother piecewise polynomials on general multilevel triangulations. It is clear to us that such wavelet bases would be very "expensive" to construct and hence are of limited practical value. However, in the case of uniform triangulations, compactly supported prewavelets and wavelet frames generated by Courant elements, or box splines, do exist and have been implemented in practice. Obviously, the $n$-term approximation from such bases or frames cannot surpass the rate of the best $n$-term Courant (or box spline) approximation, but they may give better constants and hence better performance results in practical situations.

It is also an important observation that, even in the case of uniform triangulations, the $B$-spaces used here are different from the Besov spaces used in nonlinear approximation. For a more complete discussion of this issue, see [11] and [3].

Finally, we remark that in a related paper [12] we extend the arguments of this paper to develop a corresponding approach in the Hausdorff metric which is natural for approximating surfaces. There we also consider various practical aspects for decompositions, numerical approximation, and data structures. 


\section{Appendix. Coloring Lemma}

In order to keep focus on the main analytical results of the paper, we have postponed the proof of the coloring lemma used in Section 3.2 to this appendix. This decomposition result was used to create a manageable collection of tree structures for estimating both the error and the number of elements used in our constructed approximant. Since this is a general purpose result which may prove useful in similar settings, we give its proof in full in this appendix. For clarity we have broken down the proof into a series of lemmas. Since the coloring is done in several refinement stages, it is helpful to think of the coloring as an ordered triple of primary, secondary, and shade colors. The primary coloring will sort the elements periodically by resolution level, the secondary coloring will insure there is spatial color separation, and the third coloring (shading) is a more delicate adjustment to insure that tree structures are formed. We begin by repeating the statement of the coloring lemma for the reader's convenience.

Coloring Lemma [see Lemma 3.2]. For any LR-triangulation $\mathcal{T}$ of $E$, the set $\Theta:=\Theta(\mathcal{T})$ of all cells generated by $\mathcal{T}$ can be represented as a finite disjoint union of its subsets $\left(\Theta^{\nu}\right)_{\nu=1}^{K}$ with $K=K\left(N_{0}, M_{0}\right)$ ( $N_{0}$ is the maximal valence and $M_{0}$ is the maximal number of children of a triangle in $\mathcal{T}$ ) such that each $\Theta^{\nu}$ has a tree structure with respect to the inclusion relation, i.e., if $\theta^{\prime}, \theta^{\prime \prime} \in \Theta^{\nu}$, then $\left(\theta^{\prime}\right)^{\circ} \cap\left(\theta^{\prime \prime}\right)^{\circ} \neq \emptyset$, or $\theta^{\prime} \subset \theta^{\prime \prime}$, or $\theta^{\prime \prime} \subset \theta^{\prime}$.

To begin the proof, we show, without loss of generality, that for the purposes of coloring we may assume that the multiresolution triangulation provides sufficient resolution with each refinement step. We argue below that after a certain fixed number of increments of the level there will be a guaranteed refinement of each edge and triangle, which by hypothesis is controlled from above, i.e., uniformly bounded valences and max number of subtriangles for each refinement. Consequently, we may separate the levels of $\Theta$ into $L\left(L:=\left\lceil 12 N_{0}^{4} \ln _{2} M_{0}\right\rceil\right)$ disjoint classes (primary colors) by placing two levels in the same class iff their indices are the same $(\bmod L)$. Thus a class $\tilde{\Theta}$ is of the form $\tilde{\Theta}=\bigcup_{j=0}^{\infty} \tilde{\Theta}_{j}$, where $\tilde{\Theta}_{0}:=\Theta_{j_{0}}$ for some $0 \leq j_{0}<L$ and $\tilde{\Theta}_{j}:=\Theta_{j_{0}+j L}$. Since each such class $\tilde{\Theta}$ has a different primary color, it will suffice to show how to designate the secondary colors of the members of a single $\tilde{\Theta}$. Therefore, to simplify the notation and wording of arguments, we will simply refer to (secondary) coloring the classes $\tilde{\Theta}$ instead of $\Theta$. In Lemma A.1 below we show however that these classes have additional useful properties. Loosely speaking, part (a) shows that the old vertices on a given level are far apart in terms of the graph metric. In part (b) a similar statement is given for the "central parts" of non-overlapping edges of Courant elements from different levels of $\tilde{\Theta}$.

For $D \subset \mathbb{R}^{2}$ and $m \geq 0$, we define the star $S t_{m}^{k}(D)$ inductively by $S t_{m}^{0}(D):=D$ and $S t_{m}^{k}(D):=\bigcup\left\{\theta \in \Theta\right.$ : level $\left.\theta=m, \theta^{\circ} \cap S t_{m}^{k-1}(D) \neq \emptyset\right\}$. For the vertices in resolution level $m$, this is just the neighborhood of radius $k$ in the graph metric. For an edge $e$ with vertices $v^{\prime}$ and $v^{\prime \prime}$ and an integer $m>$ level $e$, we define the "central part" of the edge to be $s t(e, m):=S t_{m}^{2}\left(e \backslash S t_{m}^{R-1}\left(\left\{v^{\prime}, v^{\prime \prime}\right\}\right)\right)$, where $R:=M_{0}^{4 N_{0}^{2}}+4$. This selection for $R$ has been made sufficiently large so that part (b) of the following lemma holds. 
Lemma A.1. The Courant collection $\tilde{\Theta}$ described above satisfies the following conditions:

(a) For each edge $\left[v, v^{\prime}\right]$ the distance between $v$ and $v^{\prime}$, measured in the graph metric on the next finer level of $\tilde{\Theta}$, is at least $4 R$.

(b) If e and $e^{\prime}$ are edges from cells in $\tilde{\Theta}, m$ is an integer with $m-L \geq$ level $e \geq$ level $e^{\prime}$, and $e \nsubseteq e^{\prime}$, then $\operatorname{st}(e, m) \cap e^{\prime}=\emptyset$.

Proof. (a) Note that each edge in $\Theta$ gets subdivided at least once after $2 N_{0}$ levels. Further, observe that after $\tilde{N}_{0}:=2 N_{0}^{2}$ refinements of any triangle, none of its vertices can be connected to their opposite edge by a single edge at the finer level. Using this observation repeatedly, one can verify that after $L$ refinements, the graph metric distance between $v$ and $v^{\prime}$ will be at least $2^{L / \tilde{N}_{0}}=M_{0}^{6 N_{0}^{2}}>4 R$.

(b) Let $v$ and $v^{\prime}$ be the vertices of $e$. Using twice the observation from the proof of part (a), we conclude that the distance from each of the vertices in $e \backslash S t_{m-2 \tilde{N}_{0}}^{1}\left(\left\{v, v^{\prime}\right\}\right)$ to $e^{\prime}$ is at least 4 when measured in the graph metric on level $m$. Therefore, on the $m$-th level, $e \backslash S t_{m-2 \tilde{N}_{0}}^{1}\left(\left\{v, v^{\prime}\right\}\right)$ has a buffer of at least three layers of triangles that separates it from $e^{\prime}$. On the other hand, the existence of $M_{0}$ and the choice of $R$ guarantee that $S t_{m}^{R-1}\left(\left\{v, v^{\prime}\right\}\right) \supset S t_{m-2 \tilde{N}_{0}}^{1}\left(\left\{v, v^{\prime}\right\}\right)$, and this establishes the claim.

This completes the primary coloring, and from this point on we only need work with a particular $\tilde{\Theta}$ (i.e., a fixed primary color). In this case "level $\theta$ " will now refer to the level of $\theta$ in $\tilde{\Theta}$ rather than in $\Theta$, as will the star $S t_{m}^{k}(\theta)$ and $s t(e, m)$. Also, when referring to the color of a cell we will now mean the secondary color, unless otherwise specified. For $\theta \in \tilde{\Theta}$ we denote by $\partial \theta$ the boundary of $\theta$, and by $x_{\theta}$ the central point of $\theta$. We say that the cells in $\Theta^{\prime} \subset \tilde{\Theta}_{m}$ are $R$-disjoint $(R \geq 1)$ if $\theta^{\circ} \cap S t_{m}^{R}\left(\theta^{\prime}\right)=\emptyset$ for any $\theta, \theta^{\prime} \in \Theta^{\prime}$.

The next result is used for the (secondary) coloring of cells of $\tilde{\Theta}$, proceeding from coarse to fine levels, and uses $M$ colors, so that same color cells are $R$-disjoint.

Lemma A.2. Suppose some of the cells on a given level are colored in $M:=$ $N_{0}^{R+1}+1(R \geq 1)$ colors so that the same color cells are $R$-disjoint. Then the rest of the cells on that level can be colored in the same $M$ colors so that the same color cells are $R$-disjoint.

Proof. To complete the coloring on the given level, we first use color \#1 to paint as many cells as possible so that the same color cells are $R$-disjoint. Next, we use color \#2 as much as possible, followed by the third and so on until either all cells get painted or we run out of colors. The latter case, however, never occurs. Indeed, assume the contrary and let $\theta$ be the first cell that cannot be colored by this algorithm with the $M$ colors. The cell $\theta$ has the property that within its $R+1$ star $S t_{m}^{R+1}(\theta)$ there must be at least one cell painted with each of the $M$ colors. But this contradicts the fact that $M$ was selected to be at least as large as the number of cells within $S t_{m}^{R+1}(\theta)$.

For the secondary coloring we proceed inductively, beginning at the coarsest level $\tilde{\Theta}_{0}$, and color cells in $M$ colors so that same color cells are $R$-disjoint. Suppose then that all levels up to $\tilde{\Theta}_{k}(k>0)$ have been colored. We color $\tilde{\Theta}_{k}$ as follows.

Step a) (Color corner cells). First we define the notion of corner cell. A cell $\theta$ of level $k$ is called a corner cell for a coarser cell $\theta^{\prime}$ if $\theta^{\prime}$ has an adjacent cell $\theta^{\prime \prime}$ (at 


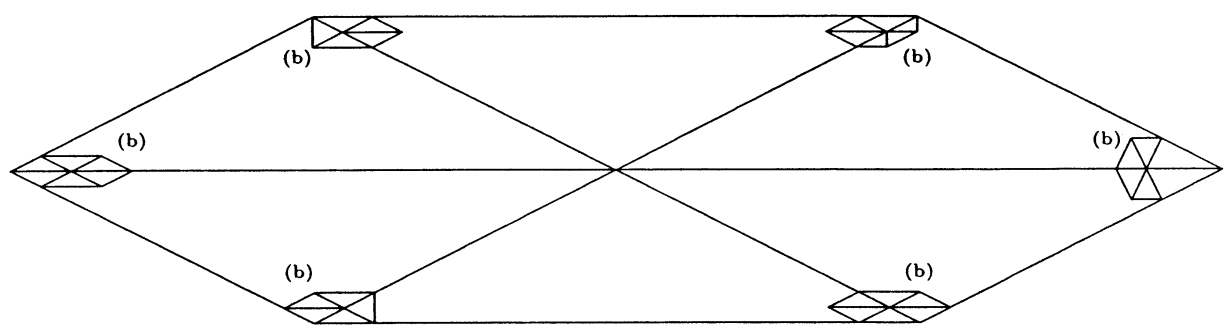

Figure 2. Corner cells from Step a)

the same level of course) so that $x_{\theta}$ lies on edge $\left[x_{\theta^{\prime}}, x_{\theta^{\prime \prime}}\right]$ and $x_{\theta}$ is adjacent to $x_{\theta^{\prime \prime}}$ on the level $k$ (see Figure 2). Given a cell $\theta^{\prime} \in \tilde{\Theta}_{k-1}$, we color each of its corner cells $\theta \in \tilde{\Theta}_{k}$ the same color as $\theta^{\prime}$. This insures that a cell's color is propagated through all finer levels to its corner cells.

Step b) (Extend the coloring to R-stars of the vertices on level $(k-1))$. For each vertex $v$ on level $(k-1)$, we paint the cells contained in $S t_{k}^{R+2}(v)$ using $M$ colors so that the coloring done in Step a) is preserved and each color is used at most once. This is always possible, since $M$ was selected sufficiently large. Note that after this step the same color cells are $R$-disjoint, since part (a) of Lemma A.1 guarantees that the stars are sufficiently separated.

Step c) (Complete the secondary coloring of $\tilde{\Theta}_{k}$ ). Accounting for the cells previously painted in Steps a) and b), we color the remaining cells from $\tilde{\Theta}_{k}$ as described in Lemma A.2.

This procedure specifies the secondary coloring of $\tilde{\Theta}$, and we have thus represented it as a finite disjoint union $\bigcup_{\nu=1}^{M} \tilde{\Theta}^{\nu}$, where $\tilde{\Theta}^{\nu}$ are all cells (secondarily) colored in the $\nu$-th color. Thus the primary color skips levels until sufficient refinement is guaranteed, while the secondary color insures sufficient spatial separation on each level to control cell overlaps. Unfortunately, the collection of same primarysecondary colored cells $\left(\tilde{\Theta}^{\nu}\right)$ might not form a tree structure, i.e., there might be two cells in $\tilde{\Theta}^{\nu}$ whose interiors meet but neither of them contains the other. This may only happen when a finer cell lies on the edge of a given cell. To fix this defect we will set for each fixed $\tilde{\Theta}^{\nu}$ the third coloring component, the shade of the cells, from two possible choices. First, we say that $\theta^{\prime}$ and $\theta^{\prime \prime}\left(\theta^{\prime}, \theta^{\prime \prime} \in \Theta\right)$ touch if an edge of the finer of the cells is contained in an edge of the coarser.

We now restrict our cells to be of fixed primary and secondary colors (i.e., fix $\tilde{\Theta}^{\nu}$ ) and inductively determine the shade of these cells. On the coarsest level $\tilde{\Theta}_{0}^{\nu}$ of $\tilde{\Theta}^{\nu}$ all cells are disjoint, and we assign them shade \#1. For the induction step, we suppose cells of all levels of $\tilde{\Theta}^{\nu}$ up to level $k$ have been shaded and each shaded collection satisfies the desired tree properties. We say that a cell $\theta$ is shade-consistent with $\tilde{\theta}$ if $x_{\theta}$ does not lie on an edge of any cell that has the same shade as $\tilde{\theta}$. Hence it is possible to place $\theta$ in this shade collection and preserve the tree structure. In this case we will also use the terminology that $\theta$ is consistent with that particular shade. We now proceed to shade the cells belonging to level $k$, i.e., $\theta \in \tilde{\Theta}_{k}^{\nu}$, according to:

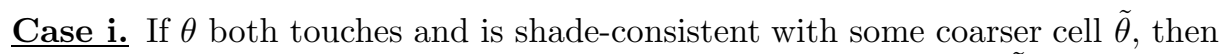
we assign to $\theta$ the same shade as that of the finest such $\tilde{\theta}$. Recall that this finest cell is unique by the construction of $\tilde{\Theta}^{\nu}$. 
Case ii. Otherwise, we assign to $\theta$ the first numbered shade for which $\theta$ is consistent. If no such shade exists, we introduce a new shade for $\theta$.

By the construction in the induction step, it is obvious that each shade subcollection has the desired tree structure. We will show that these criteria introduce at most two shades. For this we need a couple of technical facts. We remind the reader that all cells belong to a fixed $\tilde{\Theta}^{\nu}$, i.e., they have a fixed primary and secondary color.

Lemma A.3. If $\theta$ intersects an edge $e^{\prime}$ of a coarser cell $\theta^{\prime}$ but is not one of its corner cells, then $\theta \subset \operatorname{st}\left(e^{\prime}, m\right)$, where $m:=$ level $\theta$.

Proof. Let $e^{\prime}$ be an edge of $\theta^{\prime}$ that intersects $\theta$, and let $v$ be a vertex of $e^{\prime}$. By Step b) of our coloring procedure (for secondary colors), $S t_{m}^{1}(v)$ contains a corner cell $\theta^{\prime \prime}$ in $\tilde{\Theta}_{m}^{\nu}$ that is shaded the same as $\theta^{\prime}$. By Step c) in the construction of $\tilde{\Theta}^{\nu}$, $S t_{m}^{R}(v)$ does not contain any other cells from $\tilde{\Theta}_{m}^{\nu}$. Since $\theta$ is not a corner cell of $\theta^{\prime}$, then $\theta \neq \theta^{\prime \prime}$. Therefore $\theta \cap S t_{m}^{R-1}(v)=\emptyset$, and so $\theta$ must meet $e^{\prime} \backslash S t_{m}^{R-1}\left(\left\{v, v^{\prime}\right\}\right)$, where $v^{\prime}$ is the remaining vertex of $e^{\prime}$. Therefore, $\theta \subset s t\left(e^{\prime}, m\right)$.

Lemma A.4. Cells of $\tilde{\Theta}^{\nu}$ with different shades do not touch.

Proof. Suppose to the contrary that cells $\theta_{j}, \theta_{k} \in \tilde{\Theta}^{\nu}$ of different shades (shade $\# j$, shade $\# k$, respectively) do touch. We may first assume that $\theta_{j}$ is a maximal (i.e., coarsest level) cell of shade $\# j$ that touches $\theta_{k}$, and conversely, that $\theta_{k}$ is a maximal cell in shade \# $k$ that touches $\theta_{j}$. This follows by iteration and the fact that there are only finitely many coarser levels; so the iteration must terminate.

We may assume without loss of generality that level $\theta_{j}<$ level $\theta_{k}=: m_{k}$, and let $e_{j}, e_{k}$ denote the edges of $\theta_{j}, \theta_{k}$ respectively, such that $e_{k} \subset e_{j}$. We consider the two cases under which the finer cell $\theta_{k}$ could have been shaded, and show that each one leads to a contradiction.

For Case i. In this event there would be a coarser cell $\tilde{\theta}_{k} \in \tilde{\Theta}^{\nu}$ of shade \#k that touches $\theta_{k}$ and to which $\theta_{k}$ would be shade-consistent. Let $\tilde{e}_{k}$ be an edge of $\tilde{\theta}_{k}$ where it is touched by $\theta_{k}$. We consider two possible subcases, depending upon the relative level of $\tilde{\theta}_{k}$ to that of $\theta_{j}$.

Subcase i.a $\tilde{\theta}_{k}$ is finer than $\theta_{j}$.

Since level $\theta_{j} \leq$ level $\tilde{\theta}_{k}<$ level $\theta_{k}$, then by part (b) of Lemma A.1 either $\tilde{e}_{k} \subset e_{j}$ or $s t\left(\tilde{e}_{k}, m_{k}\right) \cap e_{j}=\emptyset$. The first possibility may be ruled out, since it would imply that the coarser cell $\tilde{\theta}_{k}$ would touch $\theta_{j}$, but $\theta_{k}$ is the maximal such cell of shade $\# k$. Hence $\operatorname{st}\left(\tilde{e}_{k}, m_{k}\right)$ must be disjoint from $e_{j}$. Note that $\theta_{k}$ is not a corner cell of $\tilde{\theta}_{k}$. If that were the case, then $\theta_{k}$ would be disjoint from the interiors of all edges on level $\tilde{\theta}_{k}$ except the edge on which $x_{\theta_{k}}$ lies and the edges (at most two, possibly one) where $\tilde{\theta}_{k}$ is touched by $\theta_{k}$. Hence, $e_{j}$ must overlie one of the these edges, since it contains $e_{k}$. This, however, contradicts the fact that $\theta_{j}$ touches $\theta_{k}$ in the former case and contradicts the maximality of $\theta_{k}$ in the latter. Therefore $\theta_{k}$ cannot be a corner cell of $\tilde{\theta}_{k}$, and so, by Lemma A.3 $\theta_{k} \subset \operatorname{st}\left(\tilde{e}_{k}, m_{k}\right)$. But we have already proved that $\operatorname{st}\left(\tilde{e}_{k}, m_{k}\right) \cap e_{j}=\emptyset$, which is impossible, since $\theta_{k}$ touches $\theta_{j}$ on $e_{j}$.

Subcase i.b $\tilde{\theta}_{k}$ is coarser than $\theta_{j}$.

Since level $\tilde{\theta}_{k}<$ level $\theta_{j}<$ level $\theta_{k}$, then again by part (b) of Lemma A.1 either $e_{j} \subset \tilde{e}_{k}$ or $s t\left(e_{j}, m_{k}\right) \cap \tilde{e}_{k}=\emptyset$. The former case contradicts maximality of $\theta_{k}$ relative to $\theta_{j}$. For the latter case, note that $\theta_{k}$ cannot be a corner cell of $\theta_{j}$, because $\theta_{k}$ 
and $\theta_{j}$ have different shades. Therefore, by Lemma A.3 $\theta_{k} \subset s t\left(e_{j}, m_{k}\right)$, and so we obtain $\theta_{k} \cap \tilde{e}_{k}=\emptyset$, which is impossible, since $\theta_{k}$ touches $\tilde{\theta}_{k}$ on $\tilde{e}_{k}$.

For Case ii. If this case occurred for the shading of $\theta_{k}$, then since $\theta_{j}$ is both coarser than and touches $\theta_{k}, \theta_{k}$ must not have been shade \#j consistent. Hence there must be a $\tilde{\theta}_{j} \in \tilde{\Theta}^{\nu}$ of shade $\# j$ that is coarser than $\theta_{k}$, and $x_{\theta_{k}}$ belongs to some edge $\tilde{e}_{j}$ of $\tilde{\theta}_{j}$. We consider two possible subcases, depending upon the level of $\tilde{\theta}_{j}$ relative to that of $\theta_{j}$.

Subcase ii.a $\theta_{j}$ is coarser than $\tilde{\theta}_{j}$.

Since level $\theta_{j} \leq$ level $\tilde{\theta}_{j}<$ level $\theta_{k}$, then compare edges $e_{j}, \tilde{e}_{j}$ using part (b) of Lemma A.1 to infer either $s t\left(\tilde{e}_{j}, m_{k}\right) \cap e_{j}=\emptyset$ or $\tilde{e}_{j} \subset e_{j}$. In the latter case, it follows that both the edge $e_{k}$ (recall $\theta_{k}$ touches the coarser $\theta_{j}$ on $e_{k}$ ) and the opposite vertex $x_{\theta_{k}}$ ( since $x_{\theta_{k}} \in \tilde{e}_{j}$ ) of a triangle in $\mathcal{T}_{k}$ are contained in $e_{j}$, which is clearly impossible. If the former case holds, i.e., $s t\left(\tilde{e}_{j}, m_{k}\right) \cap e_{j}=\emptyset$, then a contradiction also results. To see this, observe that $\theta_{k}$ cannot be a corner cell for $\tilde{\theta}_{j}$, due to the fact that they have different shades. But Lemma A.3 implies that $\theta_{k} \subset \operatorname{st}\left(\tilde{e}_{j}, m_{k}\right)$, which contradicts the fact that $\theta_{k} \cap e_{j} \neq \emptyset$.

$\underline{\text { Subcase ii.b }} \theta_{j}$ is finer than $\tilde{\theta}_{j}$.

Since level $\tilde{\theta}_{j}<$ level $\theta_{j}<$ level $\theta_{k}$, we again compare edges $\tilde{e}_{j}, e_{j}$ using part (b) of Lemma A.1 to imply either $s t\left(e_{j}, m_{k}\right) \cap \tilde{e}_{j}=\emptyset$ or $e_{j} \subset \tilde{e}_{j}$. By quite similar arguments to the previous subcase we can prove that contradictions are reached. Specifically, the latter statement implies that both the central vertex $x_{\theta_{k}}$ and its opposite edge $e_{k}$ belong to the edge $\tilde{e}_{j}$. On the other hand, the fact that $\theta_{k}$ cannot be a corner cell for $\theta_{j}$ will imply that $\theta_{k} \subset s t\left(e_{j}, m_{k}\right)$, which will show that $x_{\theta_{k}}$ belongs to the intersection $s t\left(e_{j}, m_{k}\right) \cap \tilde{e}_{j}$, and contradict the former statement above.

By our assumption that different shaded cells could touch, we are led in all cases to contradictions, thereby completing our contrapositive proof.

By combining the previous results with the next lemma, it follows immediately that $\Theta$ can be colored with $K:=2 M L$ colors, and the proof of the coloring lemma will be complete.

Lemma A.5. At most two shades are required.

Proof. Suppose in Case ii of the shading step above that a third shade were needed for some cell $\theta$. Then its central point $x_{\theta} \in e_{1} \cap e_{2}$ for some edges $e_{1}$ of $\theta_{1}$ and $e_{2}$ of $\theta_{2}$, where $\theta_{1}, \theta_{2} \in \tilde{\Theta}^{\nu}$ are coarser than $\theta$ and have shade $\# 1$ and shade $\# 2$, respectively. Now, if $x_{\theta}$ were a vertex for $e_{1}$, then there would be a corner cell of $\theta_{1}$ in $\tilde{\Theta}^{\nu}$ adjacent to $\theta$, which is clearly impossible, since cells at the same level are $R$-disjoint. The same reasoning applies to $e_{2}$. Therefore $x_{\theta}$ cannot be a vertex for either $e_{1}$ or $e_{2}$, and we conclude that $e_{1}^{\circ} \cap e_{2}^{\circ} \neq \emptyset$. Hence, $\theta_{1}$ and $\theta_{2}$ touch, which contradicts Lemma A.4

\section{REFERENCES}

[1] C. Bennett and R. Sharpley, Interpolation of operators, Pure and Applied Mathematics Vol. 129, Academic Press, Inc., Boston, MA, 1988. MR 89e:46001

[2] J. Bergh and J. Löfström, Interpolation spaces: An introduction, Grundlehren der Mathematischen Wissenschaften, No. 223. Springer-Verlag, Berlin-New York, 1976. MR 58:2349]

[3] O. Davydov and P. Petrushev, Nonlinear approximation from differentiable piecewise polynomials, 2002, preprint. 
[4] R. DeVore, I. Daubechies, A. Cohen, and W. Dahmen, Tree approximation and optimal encoding, Appl. Comput. Harmon. Anal. II (2001), 192-226. MR 2002g:42048

[5] R.A. DeVore, B. Jawerth, and B. Lucier, Surface compression, Computer Aided Geometric Design 9 (1992), 219-239. MR 93i:65029

[6] R.A. DeVore and G.G. Lorentz, Constructive Approximation, Grundlehren der Mathematischen Wissenschaften, Vol. 303, Springer-Verlag, Heidelberg, 1993. MR 95f:41001

[7] R.A. DeVore, P. Petrushev, and X. Yu, Nonlinear wavelet approximation in the space $C\left(R^{d}\right)$, Progress in Approximation Theory (A. A. Gonchar, E. B. Saff, eds.), Springer-Verlag, New York, 1992, pp. 261-283. MR 94h:41070

[8] R.A. DeVore and V. Popov, Interpolation of Besov spaces, Trans. Amer. Math. Soc. 305(1988), 397-414. MR 89h:46044

[9] R.A. DeVore and V. Popov, Interpolation spaces and non-linear approximation, in Function Spaces and Applications, M. Cwikel, J. Peetre, Y. Sagher, and H. Wallin (eds.), Springer Lecture Notes in Math. 1302, Springer-Verlag, Berlin, 1988, 191-205. MR 89d:41035

[10] M.A. Duchaineau, M. Wolinsky, D.E. Sigeti, M.C. Miller, C. Aldrich, and M.B. MineevWeinstein, ROAMing Terrain: Real-time Optimally Adapting Meshes, Proc. IEEE Visualization '97, October 1997, pp. 81-88.

[11] B. Karaivanov and P. Petrushev, Nonlinear piecewise polynomial approximation beyond Besov spaces, 2001, preprint. (http://www.math.sc.edu/ ${ }^{\sim}$ imip/01.html).

[12] B. Karaivanov, P. Petrushev and R.C. Sharpley, Algorithms for nonlinear piecewise polynomial approximation, 2002, preprint.

[13] P. Petrushev, Direct and converse theorems for spline and rational approximation and Besov spaces, in Function Spaces and Applications, M. Cwikel et. al. (eds), Vol. 1302 of Lecture Notes in Mathematics, Springer, Berlin, 1988, pp. 363-377. MR 89d:41027

[14] P. Petrushev, Multivariate $n$-term rational and piecewise polynomial approximation, J. Approx. Theory (2003), to appear. (http://www.math.sc.edu/ imip/01.html)

[15] P. Petrushev and V. Popov, Rational approximation of real functions, Cambridge University Press, 1987. MR 89i:41022

Department of Mathematics, University of South Carolina, Columbia, South CarOLINA 29208

E-mail address: karaivan@math.sc.edu

Department of Mathematics, University of South Carolina, Columbia, South CarOLINA 29208

E-mail address: pencho@math.sc.edu

Department of Mathematics, University of South Carolina, Columbia, South CarOLINA 29208

E-mail address: sharpley@math.sc.edu 